\author{
Universidade de São Paulo \\ Instituto de Astronomia, Geofísica e Ciências Atmosféricas
}

Departamento de Astronomia

Vinicius Branco Silva

Uma grade de espectros sintéticos para o estudo de múltiplas populações em aglomerados globulares

São Paulo 

Vinicius Branco Silva

\section{Uma grade de espectros sintéticos para o estudo de múltiplas populações em aglomerados globulares}

Dissertação apresentada ao Departamento de Astronomia do Instituto de Astronomia, Geofísica

e Ciências Atmosféricas da Universidade de São Paulo como requisito parcial para a obtenção do título de Mestre em Ciências.

Área de Concentração: Astronomia

Orientador(a):

Prof.( $\left(^{a}\right)$ Dr. (a) Paula R. T. Coelho

Versão Corrigida. O original encontra-se disponível na Unidade.

São Paulo 

Após dois anos de muito aprendizado e trabalho, um surto pandêmico surgiu e fomos obrigados a nos reinventar. Sinto pelos amigos e familiares queridos perdidos para doença e também por aqueles retirados de seus caminhos em vida. Não acredito em um lado positivo derivado desta situação, mas acredito na força para superá-la e que desta superação, sim, coisas boas podem acontecer. Dedico este trabalho a todos os sobreviventes desta pandemia, seja pela vitória fisiológica ou psicológica, e àqueles que ainda lutam: nós não estamos sozinhos!

V. Branco 



\section{Agradecimentos}

Primeiramente à minha Mãe que, além de superar seus próprios desafios, esteve ao meu lado em todos os momentos desta minha jornada, me apoiando, se preocupando, se desgastando comigo mesmo quando a luta era toda minha;

À minha família, Danillo, Pai, Ana e Soraia, pela constante preocupação quanto ao meu presente e futuro;

À minha orientadora, professora e mentora, Paula Coelho, que desde a graduação me acompanha e a quem devo muito poder concluir mais esta etapa da carreira, sempre crescendo como pessoa, aluno e melhorando para o profissional que, à semelhança dela, um dia pretendo ser;

Aos meus amigos, Cajuru, Cris, Dani, Rosi, pela compreensão e apoio, principalmente nos últimos meses de distanciamento forçado. Ao Grupo PLEAID pela disposição incansável em ajudar, mesmo quando a necessidade era a de simplesmente ter alguém para conversar;

Especialmente, ao Gê (que será mencionado devidamente ao longo da dissertação) pela paciência e por compartilhar do seu trabalho comigo e à Malu por suportar meus inúmeros questionamos em Python (quando crescer quero ser igual você) e por ambos sempre estarem lá quando precisei; 
Aos colaboradores do IAG-USP, em especial à Professora Beatriz Barbuy por prover a oportunidade de participar da LARIM 2019 e aos colegas que me acompanharam e tornaram esta uma das experiências acadêmicas mais importantes durante meu período de mestrado;

Ao Instituto de Astronomia, Geofísica e Ciências Atmosféricas, à CAPES e à Universidade de São Paulo. 
"Os objetos desta coleção são brilhantes o suficiente para serem vistos com qualquer telescópio comum, no qual parecem como cometas, ou nebulosas brilhantes, que por este disfarce, nós devemos suas descobertas a muitos eminentes astrônomos; mas de modo a verificar suas mais bonitas e artificiais construções, a aplicação de alto poder, não somente de penetração no espaço mas também de magnificação são absolutamente necessários;

E como eles são geralmente mas pouco conhecidos e são indubitavelmente os objetos mais interessantes nos céus, devo descrever vários deles (...)."

(tradução livre do excerto abaixo)

"The objects of this collection are of a sufficient brightness to be seen with any good common telescope, in which they appear like telescopic comets, or bright nebulae, and under this disguise, we owe their discovery to many eminent astronomers; but in order to ascertain their most beautiful and artificial construction, the application of high powers, not only of penetrating into space but also of magnifying are absolutely necessary; and as they are generally but little known and are undoubtedly the most interesting objects in the heavens, I shall describe several of them (...)." 



\section{Resumo}

Classicamente considerados como populações estelares simples, os Aglomerados Globulares Galácticos (AGs) são atualmente aceitos como sistemas que abrigam Múltiplas Populações de estrelas (MPs), compostas por pelo menos duas gerações: a primeira caracterizada por uma mistura "padrão" com enriquecimento de elementos- $\alpha$, observada em estrelas de campo do Halo da Via Láctea (VL); e a segunda caracterizada por um padrão de abundâncias químicas anticorrelacionadas entre CN-ONa, sobrepostas à primeira geração. Coelho et al. (2011) investigou o efeito que este fenômeno teria nas propriedades integradas das populações em um AG rico em metais como o NGC 0104 (47Tuc). Os autores concluíram que alguns índices espectrais $\left(\mathrm{CN}_{1}, \mathrm{CN}_{2}, \mathrm{Ca} 4227, \mathrm{G} 4300\right.$ e $\left.\mathrm{Na} D\right)$ são bastante afetados pelas abundâncias das anticorrelações e, além disso, que o He enriquecido na segunda geração também influencia os índices das linhas de Balmer através da mudança na temperatura de Turn-Off da população. Nosso projeto teve por objetivo expandir o trabalho de Coelho et al. (2011) aumentando a cobertura em metalicidades e comprimento de onda, de modo a melhor representar a variedade de AGs observada na VL. Para tal, construímos uma grade de espectros estelares sintéticos para a qual revisamos e utilizamos ingredientes atuais para a modelagem da atmosfera e síntese de espectro. Particularmente, a partir de uma métrica estatística, avaliamos a performance de três listas de opacidade atômica da literatura e produzimos duas novas listas, uma calibrada com o Sol e outra com Arcturus. Para calcular os modelos de atmosfera e os espectros sintéticos, desenvolvemos uma série de scripts em Python para manipular e executar em série, respectivamente, os programas ATLAS12 e SYNTHE: o PyGLOBSTERS. Com a grade de espectros estelares sintetizamos espectros integrados de três AGs usando o mesmo método de Martins et al. (2019). Avaliamos suas performances em relação aos espectros observados de Schiavon 
et al. (2005) e Usher et al. (2017) e verificamos que nossos espectros integrados em geral performaram melhor do que aqueles calculados em Martins et al. (2019). Finalmente, calculamos os índices de Lick relacionados aos efeitos das MPs e verificamos que nossos resultados corroboram o efeito não-negligível das MPs nos índices sensíveis a C, N, Ca, $\mathrm{Na}$ e os de Balmer (exceto $H \beta$ ) em NGC 0104, conforme apresentado em Coelho et al. (2011). Não obstante, notamos este efeito também em NGC 1904 e NGC 5927, que estão em outros regimes de metalicidade. Como principal resultado, verificamos, pela primeira vez, que enquanto o impacto das MPs no G4300 parece similar em metalicidades diversas, para os índices $\mathrm{CN}_{1}, \mathrm{CN}_{2}$, Ca4227 e $\mathrm{NaD}$ as diferenças se tornam maiores quanto mais rica em metal for a população estelar.

Palavras-chave: Astrofísica Estelar: Abundância Estelar; Astrofísica Estelar: Populações Estelares; Galáxia (Via Láctea): Aglomerados (Galáxia); Estrelas (individual): Sol, Arcturus; Aglomerados Globulares (individual): NGC 1904, NGC 0104, NGC 5927. 


\section{Abstract}

Classically considered as simple stellar populations, Galactic Globular Clusters (GCs) are nowadays widely accepted as systems harbouring Multiple Populations of stars (MPs), composed at least by two generations: the first characterized by a "standard" $\alpha$-enhanced metal mixture, observed in field Halo stars of the Milky Way (MW); and the second by an anti-correlated $\mathrm{CN}-\mathrm{ONa}$ chemical abundances pattern, superimposed on the first generation. Coelho et al. (2011) investigated the effect that such phenomena have on the integrated properties of populations for a metal-rich globular cluster, such as NGC 0104 (47Tuc). They found that some spectral indices $\left(\mathrm{CN}_{1}, \mathrm{CN}_{2}\right.$, Ca4227, G4300 e NaD) were appreciably affected by the abundance anti-correlations and that the enhanced He in the second generation of stars affects also the Balmer lines indices of the integrated spectra, through the change of the turn-off temperature of the population. Our project aimed at expanding that work by Coelho et al. (2011) to a larger coverage of metallicities and a wider wavelength range in order to better represent the variety of GCs observed in the MW. Hence, we built a grid of stellar synthetic spectra, reviewing and using up-to-date ingredients for the stellar atmosphere modeling and spectral synthesis. Particularly, from a statistic approach, we evaluated the performance of three atomic line lists from the literature and produced two new lists, one calibrated with the Sun and the other with Arcturus. To calculate the stellar atmosphere models and the synthetic spectra, we developed several Python scripts to manipulate and handle in a serial manner, respectively, the ATLAS12 and SYNTHE programs: the PYGLOBSTERS. Using the stellar spectra grid, we synthesized the integrated spectra of three GCs using the same method as in Martins et al. (2019). We evaluated their performances regarding the observed ones by Schiavon et al. (2005) and Usher et al. (2017) and verified that our spectra in general perform better than those 
calculated in Martins et al. (2019). Finally, we calculated the Lick indices related to the effects of the MPs and our results confirm the non-negligible effect of the MPs on indices sensitive to C, N, Ca, Na and the Balmer indices (except $H \beta$ ) for NGC 0104, as reported by Coelho et al. (2011). Additionally, we noticed this effect also in NGC 1904 and NGC 5927, which belong to other metallicity regimes. As our main result, we found, for the first time, that while the impact of the MPs on G4300 index looks similar in different metallicities, for the $\mathrm{CN}_{1}, \mathrm{CN}_{2}, \mathrm{Ca} 4227$ and $\mathrm{NaD}$ indices these differences become larger for more metal rich stellar populations.

Keywords: Stellar Astrophysics: Stellar Abundance; Stellar Astrophysics: Stellar Populations; Galaxy (Milky Way): Clusters (Galaxy); Stars (individual): Sun, Arcturus; Globular Clusters (individual): NGC 1904, NGC 0104, NGC 5927. 


\section{Lista de Figuras}

1.1 Paineis com os diagramas da Cadeia-PP e Ciclo-CNO . . . . . . . . . . 26

1.2 Paineis com os diagramas do Ciclo-NeNa e - $\mathrm{MgAl} \ldots \ldots$. . . . . . . 27

1.3 Ilustração do Diagrama HR . . . . . . . . . . . . . . . . . . . . . . 28

1.4 Ilustração do enriquecimento químico da Galáxia . . . . . . . . . . . . . 31

1.5 Aglomerado Globular por W. Herschel e HST . . . . . . . . . . . . . . . 33

1.6 Diagrama Cor-Magnitude de M3 . . . . . . . . . . . . . . . . . 34

1.7 Anticorrelação Na-O em AGs . . . . . . . . . . . . . . . . . . . . 35

1.8 Isócronas no CMD de NGC $2808 \ldots \ldots \ldots \ldots$

1.9 CMD clássico e mapa cromossômo para NGC 2808 . . . . . . . . . . . . . 37

1.10 Anticorrelação Na-O em 19 AGs observados . . . . . . . . . . . . . . . 39

1.11 Impacto das variações químicas CNONa e Y enriquecido no espectro integrado sintético de NGC $0104 \ldots \ldots$. . . . . . . . . . . . . 40

2.1 Estrelas: Sol e Arcturus . . . . . . . . . . . . . . . . . . . . . . . 42

2.2 Espectros observados do Sol e Arcturus nas regiões Ca II, $\mathrm{Mg} T$ e $\mathrm{Na} D$. . 42

2.3 Espectros telúrico observado . . . . . . . . . . . . . . . . 43

2.4 Espectro integrado de NGC 0104, observado por Schiavon. . . . . . . . . 44

2.5 Espectros observados de NGC 0104 pelas grades do WAGGS. . . . . . . . . 46

2.6 Representação de aglomerados globulares da Via Láctea . . . . . . . . . . . 47

2.7 Painel dos espectros observados de NGC 1904, NGC 0104 e NGC 5927 do WAGGS e Schiavon e seus CMDs. . . . . . . . . . . . . . . . 47

2.8 Imagens reais dos aglomerados globulares: NGC 1904, NGC 0104 e NGC 592749

3.1 Fluxograma do PyGlobsterS . . . . . . . . . . . . . . 55 
3.2 Comparação entre abundâncias químicas nos espectros sintéticos e observado do Sol de 3727 a $9300 \AA \ldots \ldots$. . . . . . . . . . . . . 59

3.3 Painel de espectros sintéticos e observado do Sol não convoluídos nas regiões de $\mathrm{CaII}[\mathrm{H}-\mathrm{K}], \operatorname{Mg} \mathrm{T}$ e $\mathrm{Na} D \ldots \ldots \ldots$

3.4 Painel comparativo das listas de opacidades moleculares $\mathrm{C}_{2}[\mathrm{D}-\mathrm{A}], \mathrm{SiH}, \mathrm{OH}$, $\mathrm{CN}[\mathrm{A}-\mathrm{X}], \mathrm{CN}[\mathrm{B}-\mathrm{X}]$ e $\mathrm{CH} . \ldots \ldots \ldots . \ldots \ldots$

3.5 Espectros sintéticos das listas de opacidade da molécula $\mathrm{C}_{2}[\mathrm{D}-\mathrm{A}] \ldots 63$

3.6 Espectros sintéticos das listas de opacidade da molécula $\mathrm{C}_{2}[\mathrm{D}-\mathrm{A}]$ em comparação ao Sol . . . . . . . . . . . . . . . . . 64 64

3.7 Espectros sintéticos das listas de opacidade da molécula $\mathrm{SiH}$. . . . . . 65

3.8 Espectros sintéticos das listas de opacidade da molécula SiH em comparação ao Sol ............................. 66

3.9 Espectros sintéticos das listas de opacidade da molécula $\mathrm{OH}$..... 67

3.10 Espectros sintéticos das listas de opacidade da molécula $\mathrm{OH}$ em comparação ao Sol .............................. 67

3.11 Espectros sintéticos das listas de opacidade da molécula $\mathrm{CN}[\mathrm{A}-\mathrm{X}]$ e $\mathrm{CN}[\mathrm{B}-\mathrm{X}] 68$

3.12 Espectros sintéticos das listas de opacidade da molécula CN [A-X] em comparação ao Sol . . . . . . . . . . . . . . . . 69 69

3.13 Espectros sintéticos das listas de opacidade da molécula $\mathrm{CN}[\mathrm{B}-\mathrm{X}]$. . . . . 70

3.14 Espectros sintéticos não-convoluídos das listas de opacidade da molécula $\mathrm{CN}[\mathrm{B}-\mathrm{X}]$ em comparação ao Sol . . . . . . . . . . . . . . . . . . . . 71

3.15 Espectros sintéticos das listas de opacidade da molécula $\mathrm{CH}$. . . . . 72

3.16 Espectros sintéticos das listas de opacidade da molécula $\mathrm{CH}$ em comparação ao Sol . . . . . . . . . . . . . . . . . . . . 72

3.17 Painel de espectros sintéticos não convoluídos da molécula $\mathrm{CH}[\mathrm{A}-\mathrm{X}], \mathrm{CH}$ [B$\mathrm{X}]$ e $\mathrm{CH}[\mathrm{C}-\mathrm{X}] \ldots \ldots \ldots \ldots$

3.18 Espectros sintéticos das listas de opacidade atômica e ADEVs calculadoss em relação ao Sol . . . . . . . . . . . . . . . . . . . . . .

3.19 Espectros sintéticos das listas de opacidade atômica e ADEVs calculados em relação a Arcturus . . . . . . . . . . . . . . . . . . .

3.20 Média ADEV entre as listas de opacidade atômica pela tolerância telúrica

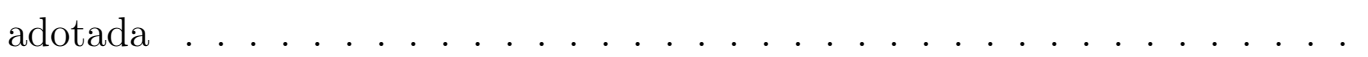


3.21 Proporção de menor ADEV das listas por intervalo $\Delta \lambda \ldots \ldots$. . . . . . 79

3.22 Diferença entre os espectros sintéticos calculados com as listas de opacidade atômica de referência e linhas "perdidas" na nova lista . . . . . . . . . . 81

3.23 Diferença entre os espectros sintéticos calculados com as listas de opacidade atômica de referência e linhas corrigidas na nova lista . . . . . . . . . .

4.1 Diagramas-HR de NGC 1904, NGC 0104 e NGC 5927 . . . . . . . . . .

4.2 Espectros sintéticos representantes da primeira e segunda geração de estrelas de NGC 0104 em comparação ao Sol e Arcturus. . . . . . . . . . . . . . . .

4.3 Espectros sintéticos da primeira e segunda gerações de estrelas de NGC 0104 nas regiões de $\mathrm{CN}$, O e Na comparados com os espectros observados do Sol e Arcturus . . . . . . . . . . . . . . . . . . . 91

4.4 Grade de espectros sintéticos estelares . . . . . . . . . . . . . . 92

5.1 Resíduos e espectros sintéticos da primeira e segunda gerações de estrelas de NGC 1904, NGC 0104 e NGC 5927 . . . . . . . . . . . . . . . .

5.2 Resíduos e espectros sintéticos da primeira e segunda gerações de estrelas de NGC 1904, NGC 0104 e NGC 5927 comparados com os espectros observados por Schiavon . . . . . . . . . . . . . . . . . . .

5.3 Resíduos e espectros sintéticos da primeira e segunda gerações de estrelas de NGC 1904, NGC 0104 e NGC 5927 comparados com os espectros observados por WAGGS . . . . . . . . . . . . . . . .

5.4 Diferença entre os espectros observados de NGC 5927 com e sem a estrela

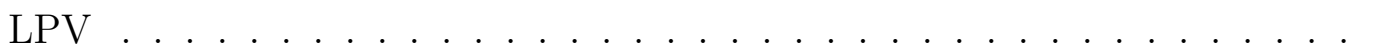

5.5 Diagramas índice-índice em função de Fe5406 com as duas gerações de estrelas para NGC 1904, NGC 0104 e NGC 5927 . . . . . . . . . . . . . 106

5.6 Diagramas índice-índice de $\mathrm{H} \beta, \mathrm{H} \delta_{F}$ e $\mathrm{H} \gamma_{F}$ em função de $\mathrm{Fe} 5406$ com as duas gerações de estrelas para NGC 1904, NGC 0104 e NGC 5927 . . . . . . 107 



\section{Lista de Tabelas}

2.1 Aglomerados globulares usados nesse trabalho e respectivas metalicidades . 45

3.1 Parâmetros atmosféricos do Sol e Arcturus . . . . . . . . . . . . . . . 53

3.2 Lista de moléculas com mais de um arquivo disponível. . . . . . . . . . . . 61

3.3 Lista de opacidades moléculares usadas no projeto . . . . . . . . . . . 74

3.4 Exemplo linhas de opacidade atômica migratórias . . . . . . . . . . . . . 81

3.5 ADEV atualizados para cada lista de opacidade atomica calibradas em relação ao Sol . . . . . . . . . . . . . . . . . . . . 83

4.1 Padrões de abundâncias químicas de cada Aglomerado Globulares . . . . . 87

4.3 Quantidade de espectros sintéticos por AG . . . . . . . . . . . . . 89

5.1 Regiões de calculo da performance ADEV dos espectros integrados sintéticos 99]

5.2 ADEV nas regiões dos Índices de Lick . . . . . . . . . . . . . . . . . . 101

5.3 Bandas características para cálculo dos Índices de Lick/IDS . . . . . . . . 103

5.4 Variação dos Índices de Lick para NGC 0104 com resolução espectral 10000104

5.5 Variações dos Índices de Lick para NGC 0104 calculadas com diferentes resoluções espectrais . . . . . . . . . . . . . . . . . . . . . . 104

B.1 Grade completa para NGC 1904 . . . . . . . . . . . . . . . . 137

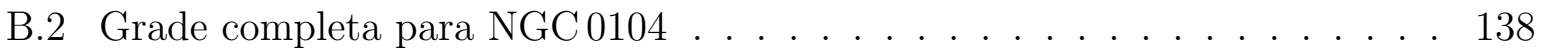

B.3 Grade completa para NGC $5927 \ldots \ldots$. . . . . . . . . . . . . . . . . . .

C.1 ADEV nas regiões dos índices de Lick (WAGGS) . . . . . . . . . . . . 141 



\section{Sumário}

1. Introdução . . . . . . . . . . . . . . . . . . . 23

1.1 Estrelas . . . . . . . . . . . . . . . . . . . . 25

1.2 Enriquecimento químico da Galáxia . . . . . . . . . . . . . . . . 30

1.3 Aglomerados globulares galácticos . . . . . . . . . . . . . . . . . . 32

1.4 Múltiplas populações de estrelas . . . . . . . . . . . . . . . 36

1.5 Anticorrelações químicas . . . . . . . . . . . . . . . . . . . . . . 38

2. Dados Observacionais . . . . . . . . . . . . . . . . . . 41

2.1 Estrelas de referência: Sol e Arcturus . . . . . . . . . . . . . . 41

2.2 Atlas de linhas telúricas . . . . . . . . . . . . . . . . . . . 43

2.3 Aglomerados globulares . . . . . . . . . . . . . . . . . . . 43

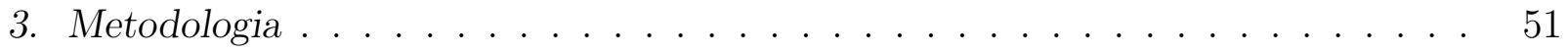

3.1 Modelos de atmosfera estelar . . . . . . . . . . . . . . . . . 51

3.2 Síntese espectral . . . . . . . . . . . . . . . . . . . 53

3.3 PyGlobsterS . . . . . . . . . . . . . . . . . . . . . . . . 54

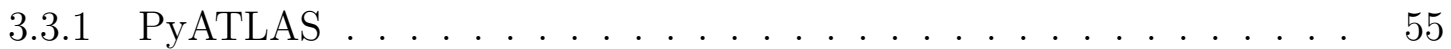

3.3 .2 PySYNTHE ....................... 57

3.4 Ingredientes da síntese espectral . . . . . . . . . . . . . . . 58

3.4 .1 Abundância solar adotada . . . . . . . . . . . 58

3.4.2 Opacidades moleculares adotadas . . . . . . . . . . 6 61

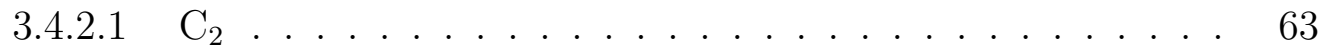

$3.4 .2 \mathrm{SiH} \ldots \ldots \ldots \ldots 6 . \ldots \ldots 6 . \ldots \ldots$

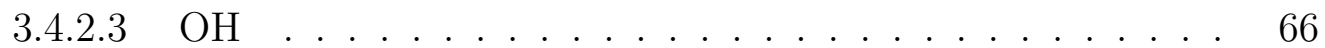




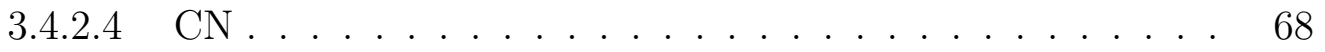

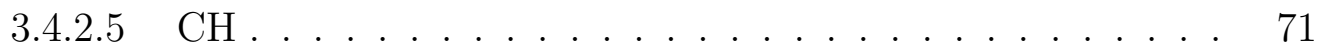

3.4.2.6 Lista final de moléculas selecionadas . . . . . . . . 74

3.4.3 Opacidades atômicas adotadas . . . . . . . . . . . . 75

3.4.4 Novas listas de opacidades atômicas . . . . . . . . . . . . . . 79 79

4. A Grade de Espectros Sintéticos . . . . . . . . . . . . . . . . . . . . . . 85

4.1 Ingredientes adotados e características gerais . . . . . . . . . . 85

4.2 Padrões de abundância química . . . . . . . . . . . . . . . . 86

4.3 Cobertura da grade em $T_{\text {eff }}, \log g$ e $[\mathrm{Fe} / \mathrm{H}] \ldots \ldots \ldots$

4.4 Espectros da grade . . . . . . . . . . . . . . . . . . . . . 89

5. Aplicação a Aglomerados Globulares Galácticos … . . . . . . . . . 93

5.1 Síntese de espectros integrados . . . . . . . . . . . . . . . . . . 93

5.2 Comparação com as observações . . . . . . . . . . . . . . . . . . 98

5.3 Comparação com a literatura . . . . . . . . . . . . . . . . . . . 102

6. Conclusões. . . . . . . . . . . . . . . . . . . . . . . . . . . . . . . 109 109

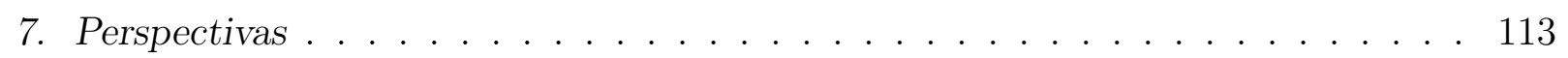

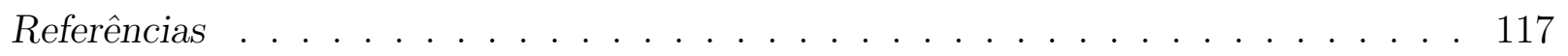

Apêndice 131

A. Abundâncias estelares . . . . . . . . . . . . . . . . . . 133

A.1 Sol . . . . . . . . . . . . . . . . . . . . . . . 133

A.1.1 Grevesse e Sauval (1998) . . . . . . . . . . . . . . . 133

A.1.2 Asplund et al. (2005) . . . . . . . . . . . . . . 134

A.1.3 Asplund et al. (2009) . . . . . . . . . . . . . . . . . 135

A.2 Arcturus . . . . . . . . . . . . . . . . . . . . . 136

A.2.1 Ramírez e Prieto (2011) . . . . . . . . . . . . . 136

B. Cobertura de parâmetros da grade . . . . . . . . . . . . . 137

B.1 NGC $1904 \ldots \ldots \ldots \ldots \ldots$ 


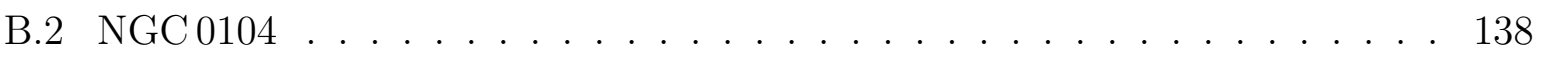

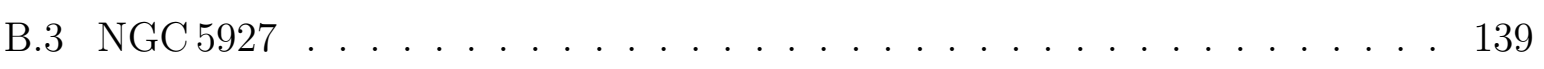

C. Comparação com as obervações … . . . . . . . . . . . . . . . . . . 141

C.1 ADEV em relação a WAGGS . . . . . . . . . . . . . . . . . . . 141 

Capítulo 1

\section{Introdução}

Historicamente, as estrelas eram elementos fixos em uma esfera no céu, agrupadas em asterismos de diferentes formas no imaginário de diversas civilizações humanas, dando sentido e guiando a vida delas. Com Galileu Galilei, em meados do século XVII, a precursionar o método científico, as estrelas passaram a pertencer a uma nova visão de mundo, desprendendo-se da abóboda celeste de outrora e sendo consideradas as responsáveis por tudo o que existe a nossa volta em termos de matéria bariônica, i.e., os prótons, nêutrons e elétrons. Com isto, os planetas, asteroides, luas, a Terra, até mesmo outras estrelas, inclusive a vida em nosso planeta 1 , são produtos de estrelas. Ou seja, quase todos os elementos da tabela períodica foram direta ou indiretamente sintetizados nas estrelas e recombinados de forma a construir o Universo que observamos hoje.

A cosmologia moderna descreve o Universo como composto majoritariamente por Hidrogênio (núcleo atômico com um próton, H), seguido de Hélio (núcleo atômico com dois prótons, He) e rastros de Lítio (Li) e Berílio (Be) (Schwarzschild, 1946), mais pesados do que eles. Da teoria da nucleossíntese primordial, momentos após o Big Bang, a colisão entre prótons, i.e., Hidrogênio, e neutrons (n) produzia um fóton $(\gamma)$ e formava um novo átomo, o Deutério $\left({ }^{2} \mathrm{H}\right)$, isótopo do Hidrogênio, conforme Equação 1.1 (veja discussão em Peebles, 2014). A colisão do Deutério com outro próton dava origem ao Hélio $\left({ }^{3} \mathrm{He}\right)$, conforme Equação 1.2, e assim por diante. Essas reações são exotérmicas, ou seja, liberam energia quando acontecem e dependem de uma temperatura necessária para que ocorram.

\footnotetext{
${ }^{1}$ E em outros...?
} 


$$
\begin{gathered}
\mathrm{H}+n \rightarrow{ }^{2} \mathrm{H}+\gamma \\
{ }^{2} \mathrm{H}+\mathrm{H} \rightarrow{ }^{3} \mathrm{He}+\gamma
\end{gathered}
$$

Estrelas são corpos celestes aproximadamente esféricos e autogravitantes capazes de realizar reações termonucleares para, no minímo, converter H em He. Além disso, elas são as responsáveis pela produção de todos os elementos mais pesados que o He, os "metais" A quantidade de Ferro produzido caracteriza a metalicidade da estrela, usualmente parametrizada por $[\mathbf{F e} / \mathbf{H}]^{3}$ (a diferença da proporção de Ferro (Fe) em relação ao H na estrela em relação à mesma proporção no Sol) ou por $\mathbf{Z}$ (a fração de massa dos elementos mais pesados que o He na estrela). Assim, a metalicidade é o grau da quantidade de elementos mais pesados do que o $\mathrm{H}$ e He. Se uma estrela tem $[\mathrm{Fe} / \mathrm{H}]=0$, então ela tem a mesma abundância de Fe presente no Sol, mas se $[\mathrm{Fe} / \mathrm{H}]=-1$, então ela tem um décimo da quantidade solar de Fe. Por tanto, quanto menor a metalicidade, menor é a razão $[\mathrm{Fe} / \mathrm{H}]$ e $\mathrm{Z} \rightarrow$ 0. Um exemplo é a estrela SMSS 1605-1443, localizada no Halo da Via Láctea, com $[\mathrm{Fe} / \mathrm{H}]=-6.2$ (Nordlander et al. 2019), sendo caracterizada como uma estrela ultra-pobre em metais.

Metalicidade é um dos atributos de uma estrela associado a muitos outros, como por exemplo à época de sua formação, quantidade inicial de massa e ao tempo de vida dela. A depender destes atributos, a estrela pertence a uma ou outra população estelar, ou seja, um conjunto de estrelas que compartilham de metalidades, composições químicas e idades muito semelhantes. Além disso, as estrelas não necessariamente estão sozinhas no Universo, constituindo sistemas de duas, três ou mais estrelas (Seção 1.1) e o ambiente no qual nascem influencia seu desenvolvimento e sua morte (Seção 1.2). Em aglomerados globulares, seja no Bojo ou Halo da Via Láctea (Seção 1.3), se discute as múltiplas populações de estrelas (Seção 1.4) e as evidências que as caracterizam (Seção 1.5).

\footnotetext{
${ }^{2}$ Em Astronomia, "metais" são todos os elementos da tabela períodica, exceto Hidrogênio e Hélio.

${ }^{3}$ Sejam os elementos "A" e "B", $[\mathrm{A} / \mathrm{B}]=\log \left(\mathrm{N}_{\mathrm{A}} / \mathrm{N}_{\mathrm{B}}\right)_{\star}-\log \left(\mathrm{N}_{\mathrm{A}} / \mathrm{N}_{\mathrm{B}}\right)_{\odot}$, onde $\mathrm{N}_{\mathrm{A}}$ e $\mathrm{N}_{\mathrm{B}}$ são as quantidades de átomos dos elementos A e B, respectivamente, na estrela $\star$ e no Sol $\odot$.
} 


\subsection{Estrelas}

As primeiras estrelas do Universo, também conhecidas como "População III" (ou primeira geração) teriam nascido em um ambiente constituído por elementos leves (i.e., H e He) e elétrons livres, ou seja, sem metais $(Z=0)$. Para observarmos hoje estrelas com maiores metalicidades, os elementos mais pesados que o $\mathrm{H}$ e He precisam ter sido produzidos dentro das estrelas ou por alguma fonte externa que as contamine (ou as duas coisas ao mesmo tempo). Estrelas que nasceram do material remanescente das primeiras estrelas do Universo constituem as estrelas da "População II". Estas estrelas de segunda geração são mais ricas em metais do que a primeira, i.e., $\mathrm{Z}>0$.

O Sol, a estrela que melhor conhecemos e representante da "População I" (ou terceira geração), por exemplo, é composto por $73,81 \%$ de $\mathrm{H}(X), 24,85 \%$ de $\mathrm{He}(Y)$ e $1,34 \%$ de metais $(Z)$ (Asplund et al., 2009), de tal modo que a soma destas frações de massa H + He + metais $=100 \%$, i.e., $X+Y+Z=1$. Assim, considerando um nascimento isolado no Universo, i.e., muito distante de outras estrelas, o Sol teria sintetizado sozinho os elementos mais pesados do que o He e/ou nasceu em um ambiente previamente contaminado para que as devidas reações termonucleares acontecessem.

O lugar no qual as estelas nascem, por exemplo se no centro ou nos cantos mais afastados de galáxias, tem influência na formação e evolução delas. A depender de sua massa inicial, a estrela será capaz de atingir temperaturas internas suficientes para ativar as reacões termonucleares que sintetizam os elementos químicos em seu interior -se não forem suficientes, não é uma estrela. Se a temperatura for alta o suficiente, cadeias e ciclos de reações termonucleares podem ser iniciados como a Cadeia-PP (ou Ciclo Próton-Próton) e o Ciclo-CNO. A Figura 1.1 (retirada de Cruz, 2012) ilustra os diagramas de cada ciclo. Em (a) e (b), as setas indicam a direção das reações. Em (b) os catalizadores são informados entre parênteses e os produtos das reações por decaimento (no caso, o decaimento- $\beta^{+}$) são informados sem parênteses. Tanto a Cadeia-PP (a) quanto o Ciclo-CNO (b) apresentam três caminhos de reações que dependem da temperatura, composisão química e densidade envolvida. Contudo, cada ciclo tem mais ou menos participação no conjunto total de reações dependendo da massa da estrela. 
Dependendo das condições termodinâmicas na estrela, outras reações termonucleares podem ser iniciadas, fundindo elementos cada vez mais pesados em outros mais pesados ainda. A Figura 1.2 (retirada de Salaris et al., 2002) ilustra o Ciclo-NeNa e -MgAl, que estão conectados pelo Sódio-23 $\left({ }^{23} \mathrm{Na}\right)$. É importante notar aqui que o Ciclo-NeNa se inicia com o $\left({ }^{20} \mathrm{Ne}\right)$, produto da reação entre o $\mathrm{O}$ e um átomo estável de He. Esta reação se chama Processo- $\alpha$ e constitui a conversão de elementos leves $4^{4}$ em elementos mais pesados através do $\mathrm{H}^{5}$. Desta forma, tal como $[\mathrm{Fe} / \mathrm{H}]$, conseguimos mensurar a abundância de elementos- $\alpha{ }^{6}$ em uma estrela a partir da relação $[\alpha / \mathrm{Fe}]^{7}$.

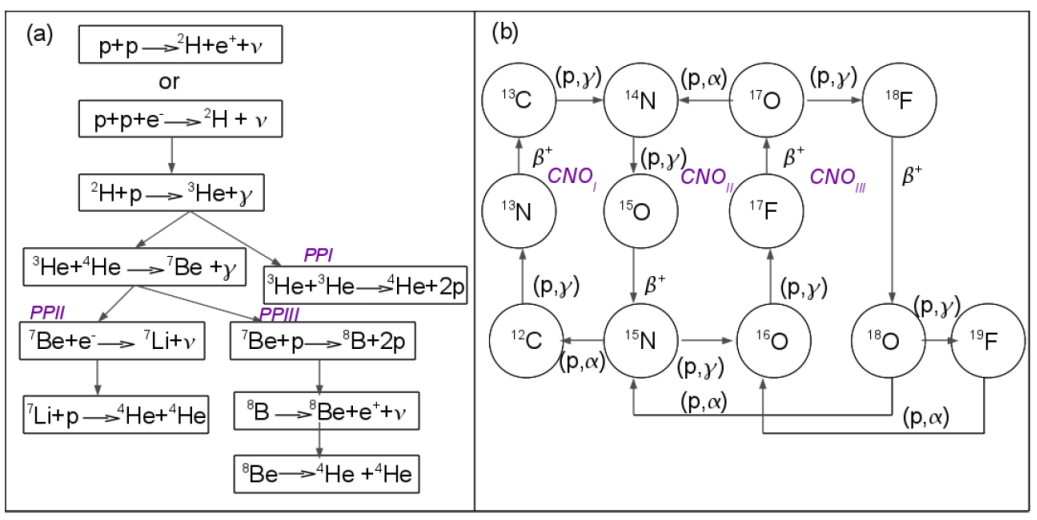

Figura 1.1: Paineis com os diagramas da Cadeia-PP (a) e Ciclo-CNO (b). As ramificações (roxo) indicam os possíveis caminhos das respectivas reações termonucleares. Imagem retirada de Cruz (2012).

\footnotetext{
${ }^{4}$ São elementos leves aqueles produzidos na nucleossíntese primordial.

${ }^{5}$ Seja o átomo A, o átomo B é produzido pelo Processo- $\alpha$ através da reação $\mathrm{A}+{ }^{4} \mathrm{He} \rightarrow \mathrm{B}+\gamma$.

${ }^{6}$ São elementos- $\alpha$ todos aqueles produzidos pelo Processo- $\alpha$.

${ }^{7}[\alpha / \mathrm{Fe}]=\log \left(\mathrm{N}_{\alpha} / \mathrm{N}_{\mathrm{Fe}}\right)_{\star}-\log \left(\mathrm{N}_{\alpha} / \mathrm{N}_{\mathrm{Fe}}\right)_{\odot}$, onde $\mathrm{N}_{\alpha}$ e $\mathrm{N}_{\mathrm{Fe}}$ são as quantidades de átomos dos elementos $\alpha$ e Fe, respectivamente, na estrela $\star$ e no Sol $\odot$.
} 


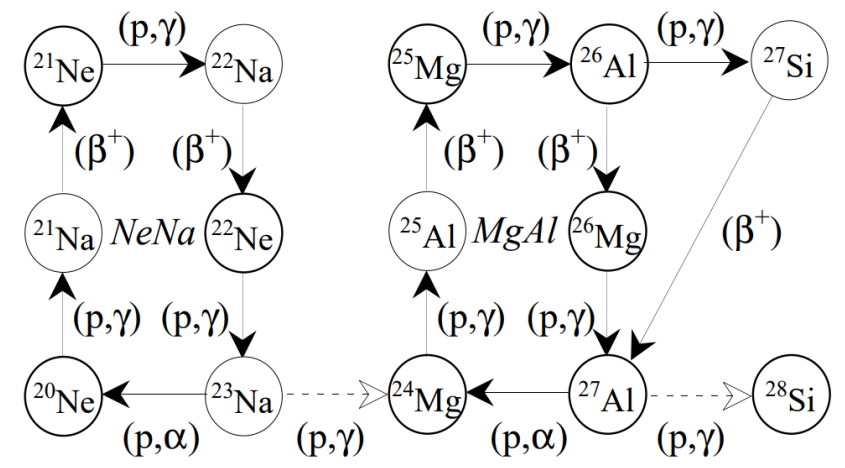

Figura 1.2: Paineis com os diagramas dos Ciclos-NeNa (esquerda) e - $\mathrm{MgAl}$ (direita). Imagem retirada de Cruz (2012).

Assim como a Cadeia-PP consome H para produzir He, o Ciclo-CNO consome H (um próton) e o Ciclo-NeNa consome He e O para serem ativados. Além disso, o C pode ser produzido através do Processo Triplo- $\alpha$, que usa três núcleos de ${ }^{4} \mathrm{He}$ : dois para produzir um átomo de ${ }^{8} \mathrm{Be}$ que reage com o terceiro para produzir C. Percebemos então que as reações estão interligadas e o produto de cada ciclo depende da eficiência deles e da abundância inicial dos elementos químicos envolvidos. Além disso, a energia necessária para a nucleossíntese estelar está relacionada com a temperatura no interior da estrela, que por sua vez depende da massa dela.

A massa das estrelas está diretamente relacionada com o brilho intrínseco delas, i.e., pela relação Massa-Luminosidade: quanto maior a massa, mais luminosa é a estrela. Como a luminosidade é diretamente proporcional ao raio da estrela (ao quadrado), quanto maior a estrela, mais luminosa ela é, também. Combinando estas relações com a temperatura efetiva estelar, constrói-se o Diagrama Hertzsprung-Russel|8 (Diagrama HR, DHR ou HRD, do inglês, "HR Diagram"). A Figura 1.3p ilustra como estrelas com diferentes luminosidades e temperaturas efetivas se dispõem em um Diagrama HR. Estrelas cujo processo de conversão do H em He está ativo em seu núcleo são localizadas na Sequência Principal (MS, do inglês, "Main Sequence").

\footnotetext{
${ }^{8}$ Criado independentemente por Ejnar Hertzsprung e Henry Norris Russell.

${ }^{9}$ Imagem copiada do livro Astronomia e Astrofísica, disponível em http://astro.if.ufrgs.br de Kepler de Souza Oliveira Filho e Maria de Fátima Oliveira Saraiva, com registros ISBN 85-7025-540-3 (2000), ISBN 85-904457-1-2 (2004) e ISBN 978-85-7861-187-3 (2013).
} 
A depender de suas massas, o caminho evolutivo de uma estrela no HR pode ser mais longo ou mais curto. Se sua massa for $\lesssim 10 M_{\odot}$, a estrela passa pelo Ramo das Gigantes Vermelhas (RGB, do inglês "Red Giant Branch"), o Ramo Horizontal (HB, do inglês "Horizontal Branch") e ascendende a seguir quase paralelamente ao RGB pelo Ramo Assimptótico das Gigantes (AGB, do inglês, "Assimptotic Giant Branch"), encerrando sua vida pacificamente em uma Anã Branca (WD, do inglês, "White Dwarf") após uma fase de nebulosa planetária. Contudo, se a massa da estrela for $\gtrsim 10 M_{\odot}$, ela passa por fases de supergigantes e encerra seu ciclo castastroficamente.

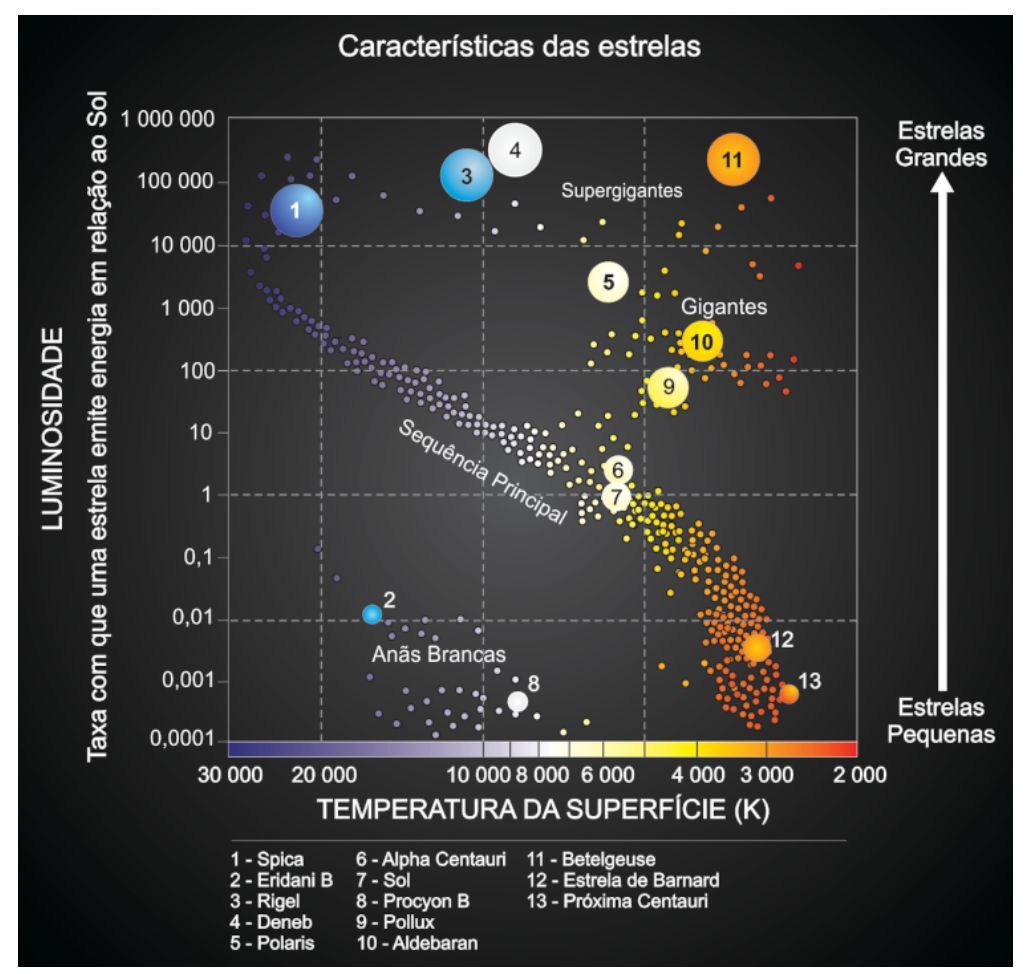

Figura 1.3: Ilustração do Diagrama HR. Crédito: de Souza Oliveira Filho e de Fatima Oliveira Saraiva (2004).

As estrelas de massa moderada, i.e., de baixa massa e massa intermediária, têm caminhos evolutivos similares, diferindo principalmente da fonte energética entre elas na MS. Estrelas de baixa massa tem como principal fonte de energia a Cadeia-PP, ao passo que o Ciclo-CNO domina em estrelas de massa intermediária. Assim, apesar de o fim da vida das estrelas destas categorias serem semelhantes, as de massa intermediária experimentam processos de mistura química muito mais complexos do que as de baixa massa (Karakas e Lattanzio, 2014). 
No topo do AGB de estrelas de massa intermediária, a TP-AGB (do inglês, "Thermally Pulsing-AGB", pulso térmico-AGB) representa uma fase de instabilidade na qual as camadas de queima de $\mathrm{H}$ e He se intercalam quanto ao domínio de energia produzida pela estrela. Em cada pulso térmico, a base da camada convectiva, que está envolta ao núcleo inerte de $\mathrm{C}-\mathrm{O}$, se move para regiões mais profundas da estrela, levando os produtos das queimas de H e He para a superfície (terceira dragagem). Além disso, a cada pulso há perda de material químico que é jogado para o meio interestelar. Para estrelas com massa $\gtrsim 3 M_{\odot}$ (Ventura et al. 2020), a cada pulso térmico da TP-AGB, acontece um evento conhecido como Hot Bottom Burning (HBB, "queima quente da base", em tradução livre) no qual os Ciclos-NeNa e -MgAl são ativados e parte de seus produtos é levado para a superfície pela terceira dragagem. E assim, ao invés de se encerrar em uma WD com núcleo degenerado de C-O, seu núcleo será composto de ONeMg degenerado (Cruz, 2012, e referências).

Já as estrelas massivas não experimentam a fase AGB. Elas ascendem ao ramo das Gigantes fundindo He em seu núcleo com as camadas convectivas dominando a produção energética. Em comparação às estrelas de massa moderada, nas de alta massa há apenas a primeira dragagem, contudo elas também passam pelo HBB, ainda na MS. As temperaturas nas zonas convectivas das estrelas massivas são suficientes para ativar os Ciclos-CNO e -NeNa com muito mais eficiência do que sua contrapartida menos massiva, de modo que até o Ciclo-MgAl pode ser ativado (Decressin et al., 2007). Além disso, a alta rotação contribui para que as camadas convectivas esgotem os recursos mais eficientemente, expulsando o material dragado para o meio interestelar.

Ao fim da vida de uma estrela massiva, quando a pressão devido à gravidade se torna mais forte do que a pressão de radiação (i.e., as reações termonucleares no núcleo estelar cessam), ela se encerra colapsando seu núcleo violentamente em um evento catastrófico conhecido como Supernova Tipo II, ou SN II (entre outros tipos de supernova de colapso de núcleo; veja o artigo de revisão por Pols, 1997). O remanescente da explosão depende da sua massa inicial, deixando um núcleo composto por nêutrons degenerados (i.e., Estrela de Nêutrons) ou um Buraco Negro. Esta explosão faz com que todo material externo ao núcleo seja expelido para o meio interestelar. 


\subsection{Enriquecimento químico da Galáxia}

À semelhança das estrelas, que se formam do acúmulo de matéria pela força da gravidade, galáxias se constituem de dezenas a centenas de milhares de estrelas. A depender da história de formação e evolução delas, as galáxias possuem diferentes propriedades morfológicas, químicas e dinâmicas. A Via Láctea (VL ou MW, do inglês, "Milky Way"), por exemplo, é constituída por um Disco Fino e outro espesso, e um Bojo com barra, considerada uma galáxia espiral fracamente barrada.

Cada uma destas estruturas da VL contém grupos de estrelas que, por sua vez, constituem populações estelares. O bojo, que envolve o centro Galáctico, é composto por estrelas jovens e velhas, ricas e pobres em metais, de modo que ainda está em debate como as idades e metalicidades das estrelas se distribuem (veja Seções 3.1 e 3.2 do review de Barbuy et al., 2018). O disco fino é a região que abriga as estrelas mais jovens da Galáxia e também abriga a maior parte do gás e poeira remanescentes. E o Halo, por sua vez, é o asilo Galáctico, composto com estrelas velhas e muito pobres em metais.

Como vimos anteriormente, parte do material processado dentro das estrelas é jogado para fora durante sua evolução, contaminando o meio interestelar. Este material expulso pode se acumular e formar uma nova estrela, aos moldes de sua antecessora. Contudo, ela já nasce com uma abundância em metais diferentes (e maior). Assim, as gerações de estrelas em uma galáxia se diferenciam pelo enriquecimento químico que suas progenitoras proporcionaram.

Além das SN II, como vimos na seção anterior, existem as SN Ia que acontecem, essencialmente, quando estrelas de massa moderada (i.e., $\lesssim 10 M_{\odot}$ ) possuem uma estrela companheira mais massiva do que elas, formando um sistema binário. Com o passar do tempo, a estrela menos massiva evolui para uma WD e, devido sua alta densidade que aumenta sua força gravitacional, interage com as camadas externas da sua companheira, atraindo o material dela pra si (agora, este é um sistema binário interagente, ou IB, do 
inglês, "Interacting Binary"). Com o acúmulo de massa, a degenerescência de seus núcleo de C-O é quebrada e a WD pode explodir em Supernova Tipo Ia (SN Ia), enriquecendo o meio interestelar com elementos do pico do Ferro.

A Figura 1.4 (imagem adaptada do review por Chiappini, 2001) ilustra como a abundância de elementos- $\alpha$ (representados na figura pelo Oxigênio) pode ter mudado no tempo em relação à metalicidade geral da Galáxia para estrelas de campo (i.e., as estrelas isoladas no meio interestelar). Para metalicidades menores que $[\mathrm{Fe} / \mathrm{H}]=-1,0$, tanto o $\mathrm{O}$ como Fe são produzidos principalmente em Supernovas tipo II por estrelas no Halo e Disco Espesso da Galáxia (em vermelho e verde, respectivamente), dando origem a um "plateau" na razão [O/Fe]. Quando as estrelas do Disco Fino explodem em Supernovas Tipo Ia, a proporção de Fe na Galaxia aumenta e a razão $[\mathrm{Fe} / \mathrm{H}]$ cai (em azul), dando origem ao que se identificada na Figura 1.4 por "joelho".

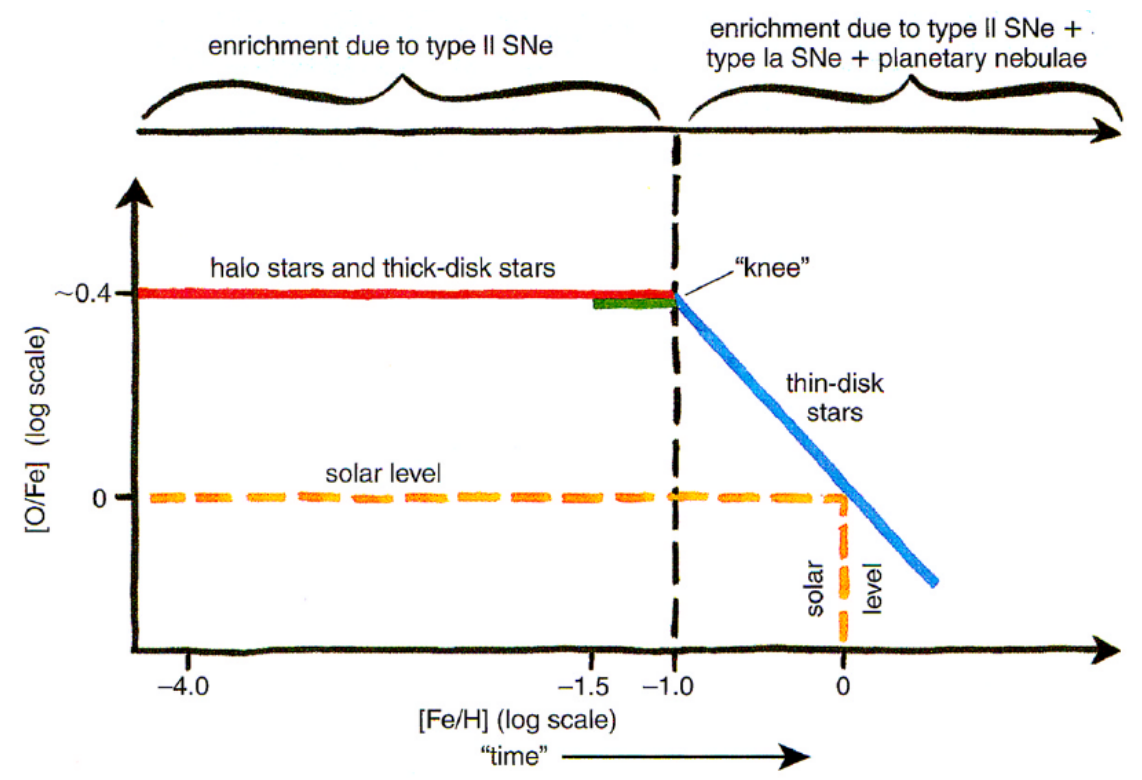

Figura 1.4: Esquema geral da abundância relativa de oxigênio [O/Fe] em função da metalicidade [Fe/H] para estrelas de campo da Via Láctea. A linha tracejada indica as abundâncias relativas ao Sol (veja o review por Chiappini, 2001, para mais detalhes). Crédito: Chiappini (2001). 


\subsection{Aglomerados globulares galácticos}

Sobre uma de suas "nebulosas globulares", como observados por Sir John Frederick William Herschel, ele disse: "é um aglomerado de estrelas com uma imagem redonda, mas as estrelas fracas fora dos aglomerados globulares estão geralmente um pouco dispersas de modo a desviarem da forma cirular perfeita"; "Ele é gradual e extremamente condensado no centro, mas com mais atenção, mesmo ali, as estrelas são distinguíveis"; "Há muitas estrelas no campo de visão, mas elas são muitas ordens de magnitudes totalmente diferentes daquelas excessivamente menores que compõem o aglomerado"; "Não é possível ter uma ideia do número de estrelas em tal aglomerado, mas eu penso que não podemos estimar às centenas" (excertos de Herschel, 1814, página 274, em tradução livre).

Da época de Sir Herschel até hoje, foi aumentado o poder dos telescópios, e as "nebulosas globulares" de antes correspondem aos Aglomerados Globulares (AGs ou GCs, do inglês, "Globular Clusters de hoje, definidos como sistemas compactos de dezenas a centenas de milhares de estrelas gravitacionalmente ligadas. AGs estão presentes principalmente no Bojo e no Halo da VL, com magnitude visual absoluta média de $M_{V} \sim-7$ (Gratton et al. 2019), sendo um dos artefatos astrofísicos mais antigos de galáxias, podendo ter tido um papel importante na formação e evolução delas (Renzini, 2017). A Figura 1.5 coloca lado a lado a ilustração publicada por Herschel sobre o AG ao qual ele se referia nos excertos acima e a imagem de NGC 0104 obtida com telescópios modernos, $\sim 200$ anos depois. 

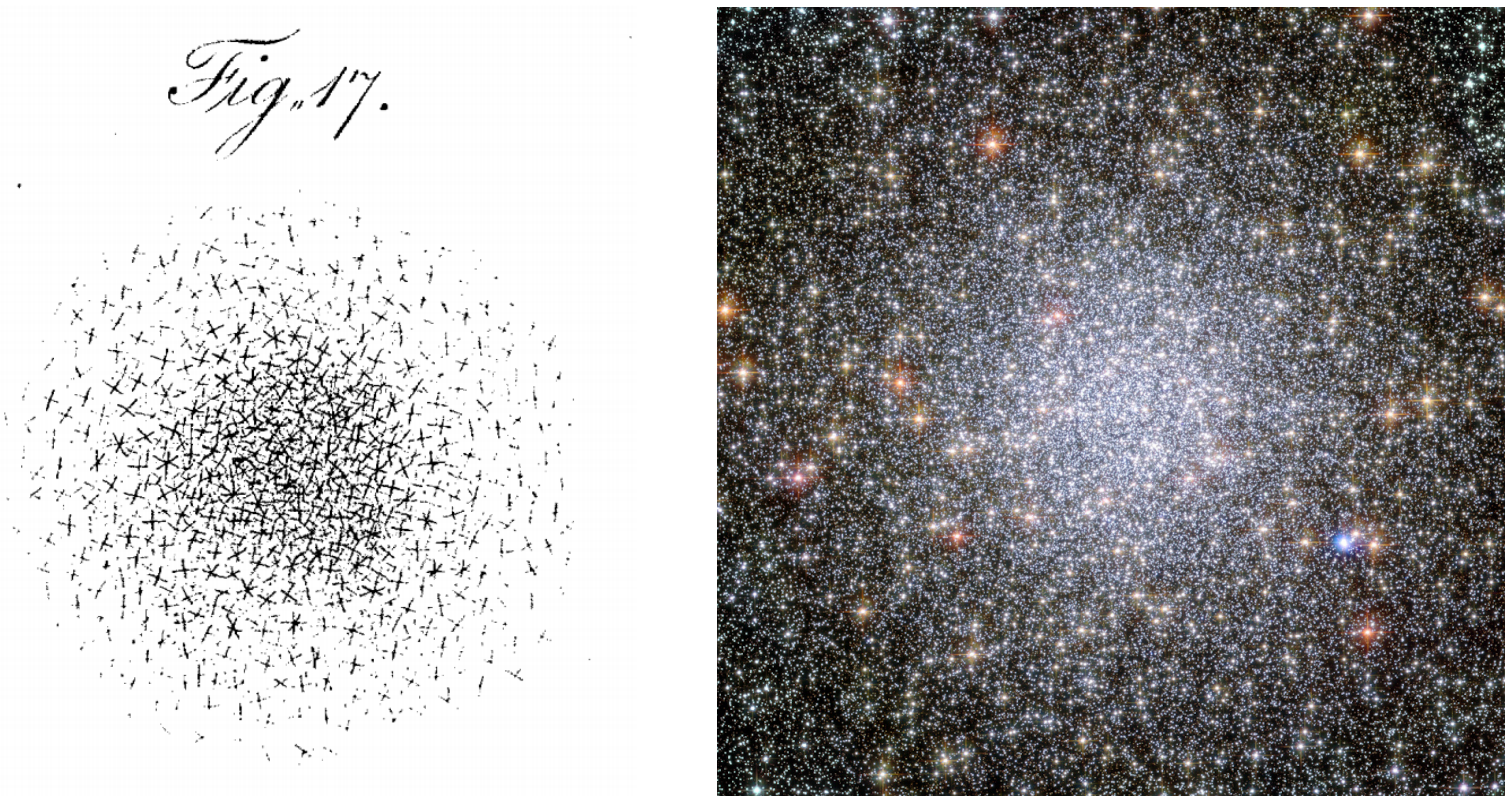

Figura 1.5: Esquerda: Ilustração retirada de Herschel (1814) referente ao AG descrito por ele em sua época (veja no texto). Direita: Fotografia moderna de um AG: NGC0104 (47 Tucanae) (NASA/ESA/STScI/AURA/ESA-HST, 2015).

Classicamente, desde a descoberta dos aglomerados globulares até o início dos anos 2000, as estrelas pertencentes a um mesmo AG eram consideradas como sendo uma população estelar simples (SSP, do inglês, "Simple Stellar Population"). Uma SSP assume que suas estrelas compartilham da mesma idade e composição química inicial, com apenas as massas diferindo conforme a Função de Massa Inicial (IMF, do inglês "Initial Mass Function"). Com isto, as estrelas teriam coevoluído a partir da mesma nuvem molecular, todas possuindo a mesma metalicidade, sendo a única diferença entre elas as abundâncias químicas relativas ao estágio evolutivo em que estão.

Por muito tempo, esta ideia foi corroborada por observações fotométricas que indicavam que os AGs abrigavam somente uma SSP. A partir do Diagrama Cor-Magnitude (CMD, do inglês, "Color-Magnitude Diagram"), as previsões teóricas podiam ser diretamente comparadas com as estrelas observadas. A Figura 1.6 (retirada de Sandage, 1970) ilustra uma tentativa de representar o aglomerado M3 no CMD. A linha cheia é conhecida como isócrona (do grego "iso", igual; e "cronos", tempo - ou "mesmo tempo", i.e., mesma idade) e, portanto, se supõe que nela estão localizadas todas as estrelas que nasceram ao mesmo tempo, ou seja, uma SSP. 


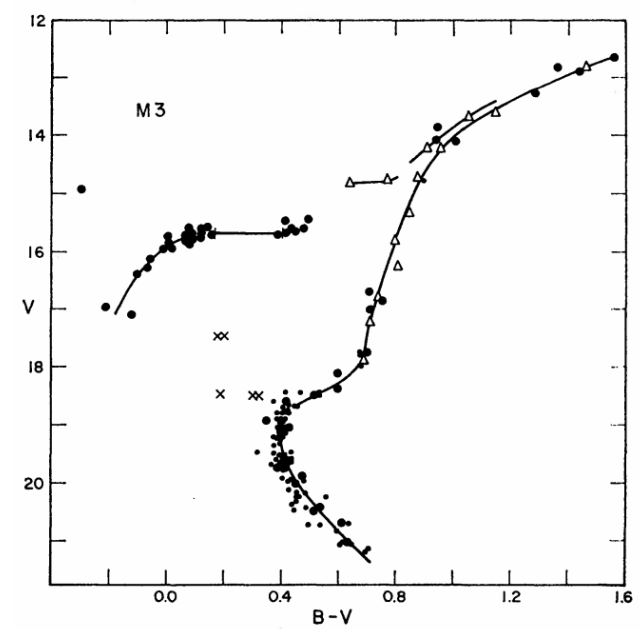

Figura 1.6: Diagrama Cor-Magnitude (CMD) de M3. Crédito: Sandage 1970).

Contudo, em meados dos anos 1990, a partir de medidas espectroscópicas, alguns estudos já apontavam para discrepâncias encontradas nas abundâncias químicas entre estrelas observadas de AGs e estrelas de campo do Halo (Wheeler et al., 1989; Kraft, 1994). Por exemplo, no estudo de estrelas gigantes vermelhas do aglomerado globular M13 foram encontradas anticorrelações entre $\mathrm{Na}$ e $\mathrm{O}$ e entre $\mathrm{Mg}$ e $\mathrm{Al}$, não encontradas em estrelas de campo no Halo Galáctico. A Figura 1.7 (retirada de Kraft et al., 1997) mostra a anticorrelação encontrada entre as abundâncas de $\mathrm{Na}$ e $\mathrm{O}$ em relação ao Fe nas gigantes vermelhas de seis aglomerados globulares (indicados na legenda da figura). Diferenças nas abundâncias de C, N, O, Na, Mg e Al em AGs motivaram outros estudos a buscar por processos que pudessem explicar as discrepâncias encontradas (veja série de artigos por Kraft et al., 1997). 


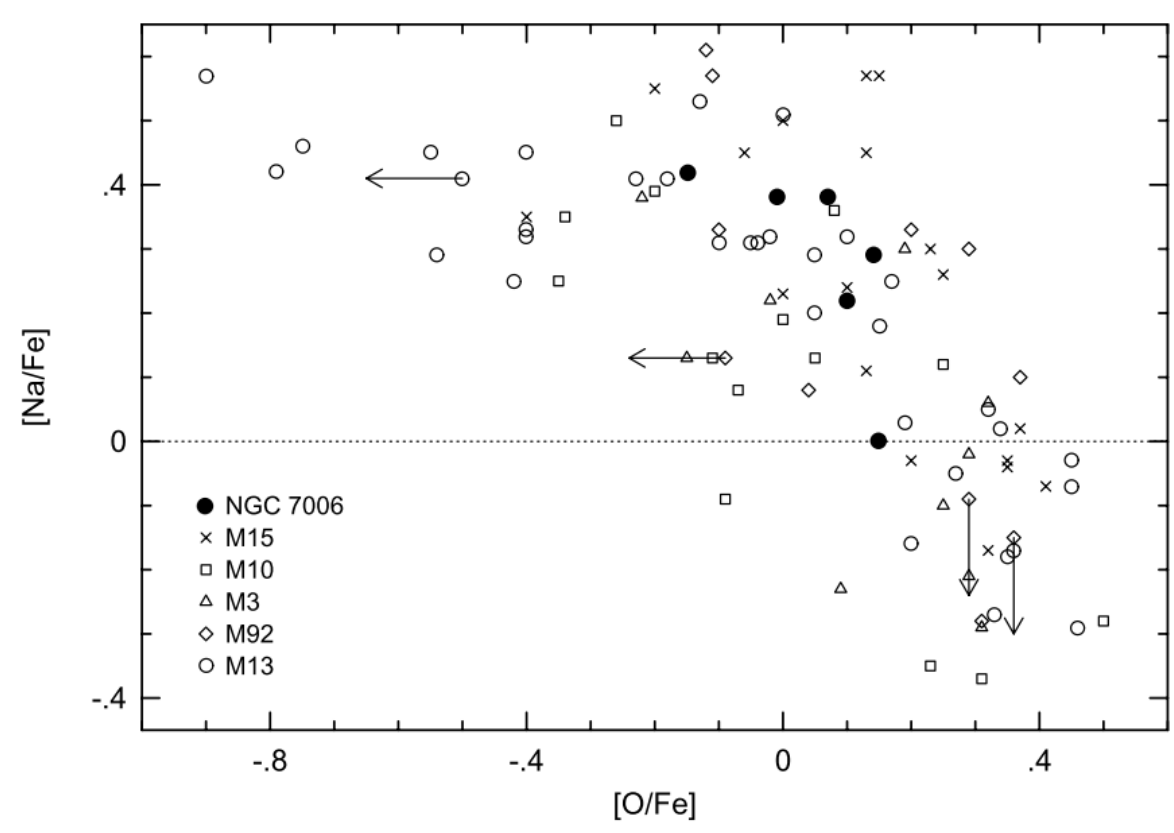

Figura 1.7: Anticorrelação Na-O para diversos AGs (legenda da figura). Crédito: Kraft et al. (1997).

Para tentar explicar as anticorrelações entre as estrelas gigantes dos AGs (Denissenkov e Weiss, 1996), Denissenkov e Weiss (2001) apresentaram o que foi chamado de "cenário de mistura profunda", um processo de mistura adicional entre o HBB e a camada de queima do $\mathrm{H}$ na fase RGB. No entanto, foram encontradas evidências das anticorrelações Na-O (e outras) em estrelas da sequência principal, até então esperadas somente nas estrelas do RGB. Isto levantou a questão de que mecanismos internos das estrelas de baixa massa da MS talvez não fossem suficientes para explicar as discrepâncias químicas encontradas entre estrelas de um mesmo AG.

Com medidas fotométricas e espectroscópicas cada vez mais precisas e de maior resolução observacional, a ideia de que AGs fossem compostos de uma SSP foi colocada em dúvida: múltiplas sequências principais começaram a ser reveladas (Piotto et al., 2007). A Figura 1.8 ilustra uma evidência fotométrica para NGC 2808, na qual quatro isócronas com hipóteses de fração de massa em He $(\mathbf{Y})$ diferentes entre si (linhas coloridas) foram usadas para tentar representar a MS estendida que se observou neste AG. A necessidade de se usar mais de uma isócrona para representar o CMD de alguns aglomerados levou o estudo a um novo paradigma: o de múltiplas gerações de estrelas em AGs. 


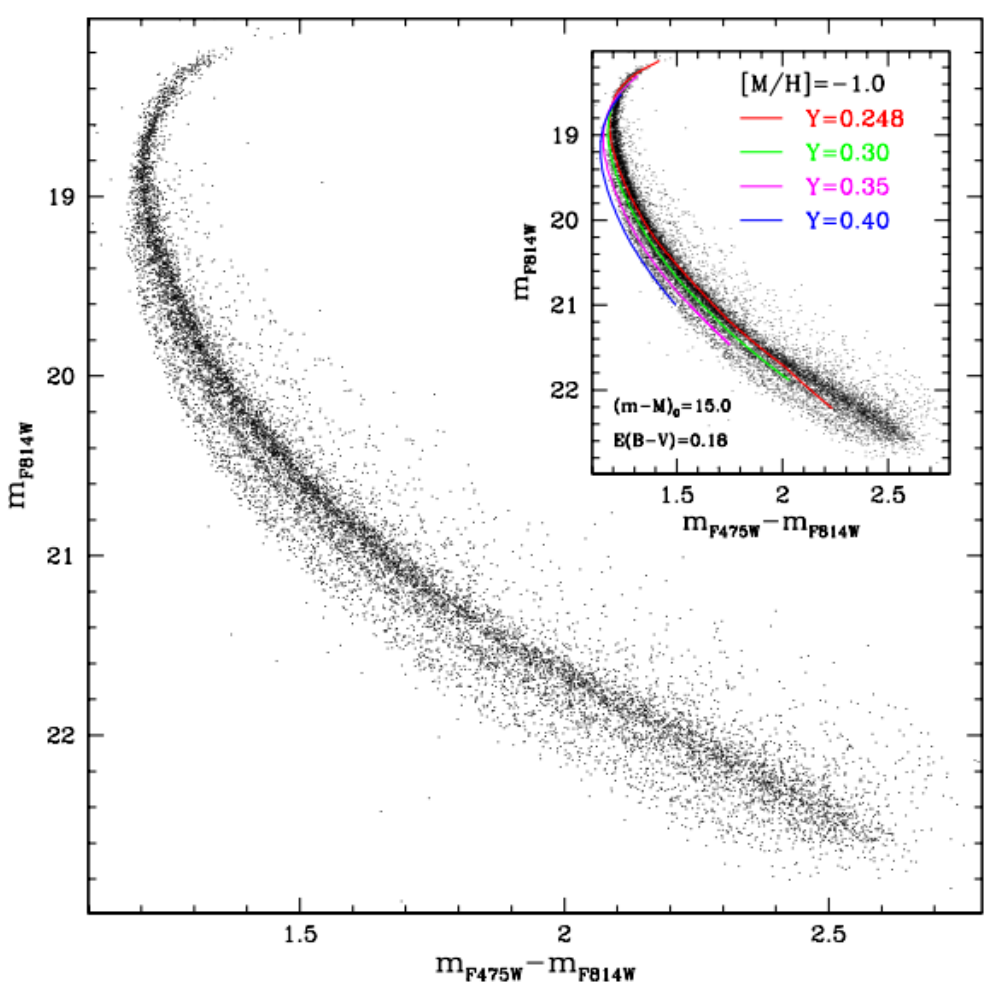

Figura 1.8: Ajuste de quatro isócronas na MS para o aglomerado globular NGC 2808. Crédito: Piotto et al. (2007).

\subsection{Múltiplas populações de estrelas}

Na última década, as evidências observacionais, tanto fotométricas com o Hubble Space Telescopy (HST) quanto espectroscópicas com o Very Large Telescope (VLT), apontavam cada mais na direção de que aglomerados globulares não podiam ser mais entendidos como sistemas de estrelas com uma única geração (veja review por Gratton et al., 2019). Desta forma, as estrelas em um dado aglomerado podem ser separadas em diferentes gerações, com a mais nova carregando o material químico deixado no meio interestelar, poluído por gerações anteriores.

Com isso, AGs que apresentam múltiplas gerações de estelas possuem, portanto, Múltiplas Populações (MPs, do inglês, "Multiple Populations"), cada uma com seu padrão próprio de abundâncias químicas. Tipicamente a segunda geração de estrelas apresenta variações nas abundâncias químicas dos elementos leves que não são esperadas pelos processos de reação termonuclear da teoria de evolução estelar. A Figura 1.9 (retirada do review por Bastian 
e Lardo, 2018) ilustra, em contraste à Figura 1.8, outra forma de se verificar as MPs em AGs: o "mapa cromossômo", que separa as gerações do AG a partir da combinação específica entre três filtros do Hubble Space Telescopy (HST), o "trio mágico", como chamado pelos autores. Enquanto o CMD clássico aponta para duas ou três populações no RGB de NGC 2808, o "mapa cromossômo" infere subpopulações no CMD modificado, partindo de diferentes hipóstes de mistura química, i.e., variando abundâncias de $\mathrm{N}$ e fração de massa Y.
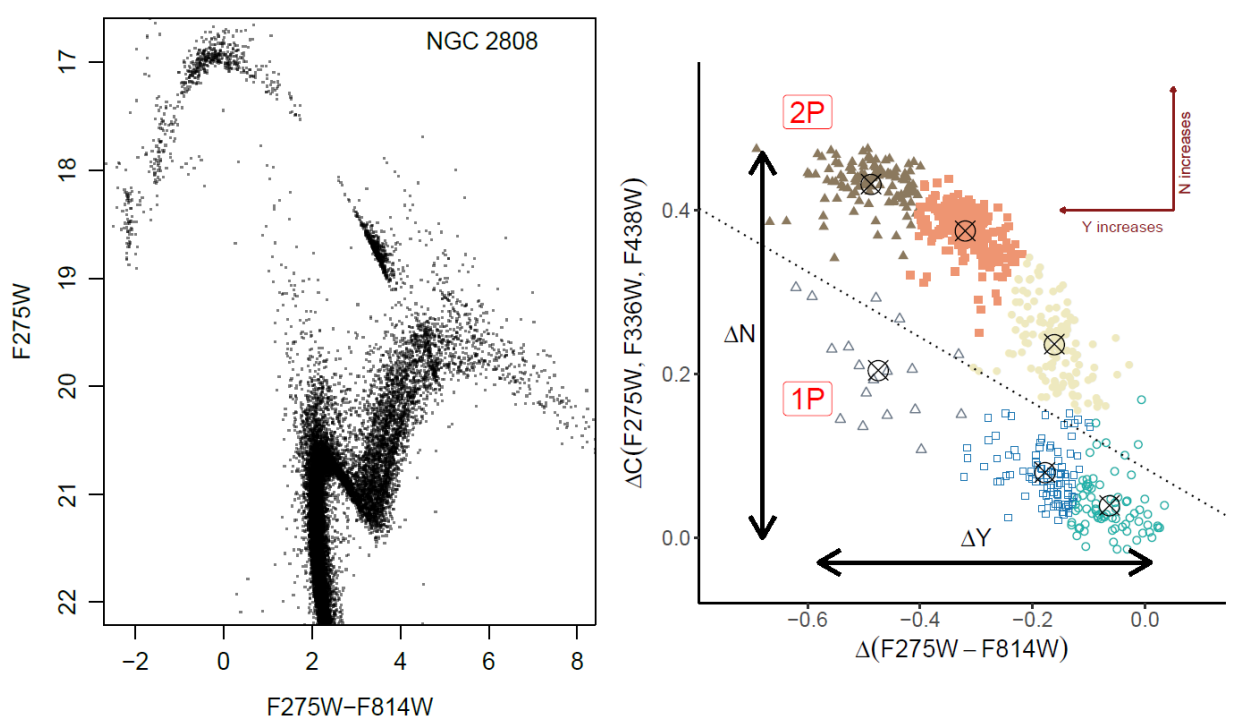

Figura 1.9: Esquerda: CMD distinguindo possíveis populações no RGB para NGC 2808 com dois filtros do HST por Piotto et al. (2015); direita: seis possíveis subpopulações (regiões coloridas) inferidas para o mesmo AG com o "mapa cromossômo" por Milone et al. (2016).

No sentido de que uma parte das estrelas de dado AG deve nascer em um meio contaminado quimicamente por uma geração anterior do mesmo AG, e de acordo com o compilado no review por Bastian e Lardo (2018), são candidatos a poluidores do meio intraaglomeradq $q^{10}$; estrelas massivas $\left(>15 M_{\odot}\right)$; estrelas extremamente massivas $\left(\sim 10^{4} M_{\odot}\right)$; estrelas AGB (de massa intermediária; $5-6.5 M_{\odot}$; estrelas massivas de alta rotação (FRMS, do inglês, "Fast Rotating Massive Stars"); e as estrelas binárias interagentes (IBs). Em cada cenário proposto é feita a tentativa de contabilizar pelas quantidades químicas medidas nos AGs, porém, segundo a literatura, apesar de cada um ter sua vantagem e desvantagem, sozinhos não têm sido capazes de explicar o paradigma por completo.

\footnotetext{
${ }^{10}$ Veja o capítulo 4 do review por Bastian e Lardo (2018) para outros cenários.
} 


\subsection{Anticorrelações químicas}

Aglomerados Globulares são empregados como traçadores da história de formação estelar em sistemas dinamicamente quentes (e.g., veja o artigo de revisão por Brodie e Strader. 2006). Em estudos extragalácticos seus espectros ainda são tipicamente modelados considerando apenas uma SSP. Esses espectros são considerados integrados, por serem na prática resultado da contribuição dos espectros estelares de suas estrelas. Assim, os espectros integrados de AGs são geralmente utilizados para se testar modelos de SSPs (e.g., veja Lee e Worthey, 2005; Percival et al., 2009; Walcher et al., 2009; Vazdekis et al., 2010; Thomas et al., 2011) e a interpretação destas comparações dependem do efeito das abundâncias das anticorrelações no espectro do aglomerado.

As evidências fotométricas, como as que apresentamos na seção anterior, demonstraram que as MPs em AGs podem ser identificadas nos CMDs e, mais ainda, caracterizadas em subpopulações (Milone et al., 2016). Contudo, do ponto de vista espectroscópico, em um dado AG, as quantidades químicas podem ser medidas estrela a estrela, desde que as estrelas sejam resolvidas pelo telescópio (ou seja, discriminadas individualmente). Carretta et al. (2009b) mediram uma das principais anticorrelações químicas características das MPs: o Na-O. A Figura 1.10 (retirada de Carretta et al., 2010) apresenta as anticorrelações das abundâncias de $\mathrm{Na}$ e $\mathrm{O}$ medidas para 19 AGs da Galáxia. Além do Na-O, foram demonstradas outras anticorrelações características das MPs como entre C e N, Mg e $\mathrm{Al}$ (Carretta et al. 2018). Outra característica das inomogeniedades químicas encontradas está relacionada ao enriquecimento da abundância inicial da fração de He (Y) (D’Ercole et al., 2008, 2010; Milone et al., 2018), como mostraram as evidências fotométricas para NGC 2808 (seção anterior).

O primeiro trabalho a explorar como essas variações químicas impactaria a previsão da luz integrada de AGs foi o de Coelho et al. (2011). Naquele trabalho, as variações químicas observadas em C, N, O, Na e na fração de massa Y (i.e., de He) foram usadas na modelagem de duas gerações de espectros integrados. Uma população foi caracterizada com as abundâncias químicas características das estrelas de campo do halo, com $[\alpha / \mathrm{Fe}]$ $=0,4$ e $\mathrm{Y}=0,256$, e a outra população considerava as abundâncias CNONa conforme 


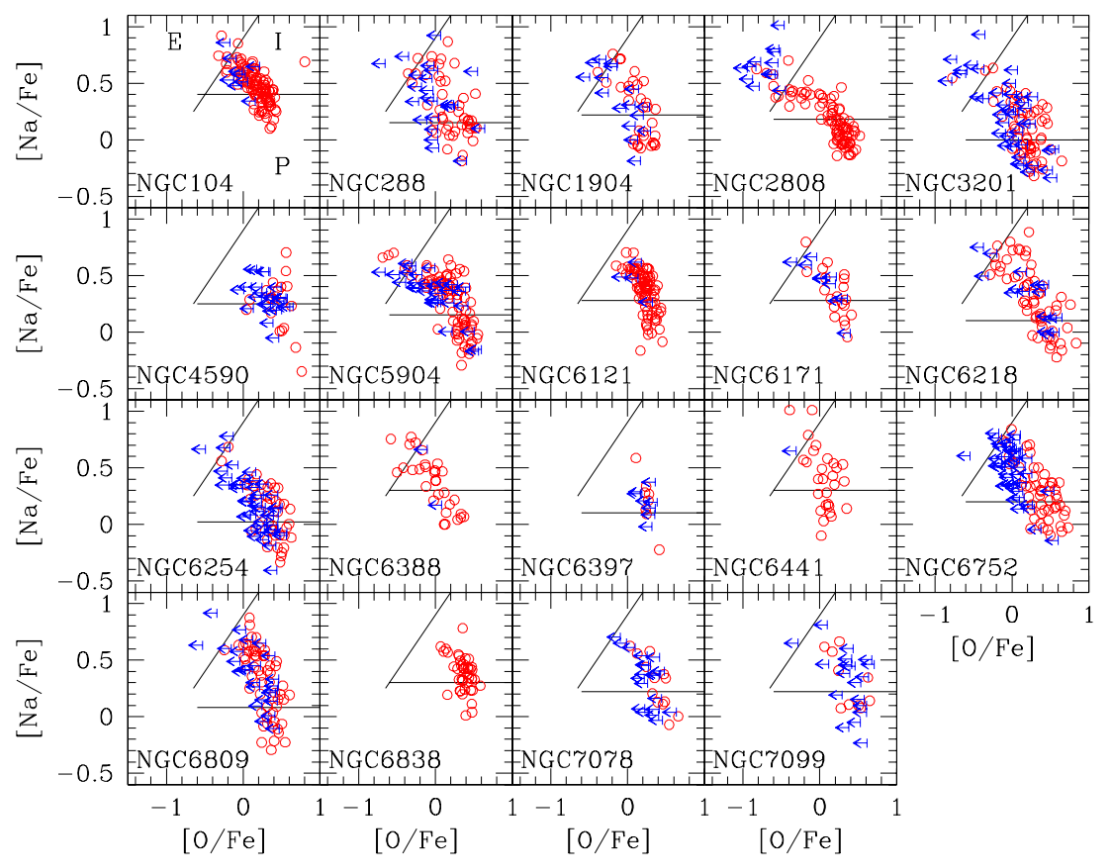

Figura 1.10: Anticorrelação Na-O em 19 AGs observados por Carretta et al. (2010). As setas azuis indicam limites superiores das abundâncias de $\mathrm{O}$ e as linhas pretas separam as populações estelares em cada AG, sendo uma extremamente pobre em $\mathrm{O}$ e rica em $\mathrm{Na}(\mathrm{E})$, uma extremamente rica em $\mathrm{O}$ e pobre em $\mathrm{Na}(\mathrm{P})$ e outra intermediária no meio (I), conforme primeiro painel superior esquerdo. Crédito: Carretta et al. (2010).

$\Delta(\mathrm{C}, \mathrm{N}, \mathrm{O}, \mathrm{Na})=\Delta(-0.30,+1.20,-0.45,+0.60)$ e $\mathrm{Y}=0,300-$ valores superiores típicos encontrados na literatura (Coelho et al., 2011, 2012), sendo que a soma em massa de $\mathrm{C}+\mathrm{N}+\mathrm{O}$ é constante entre as misturas (Gratton et al., 2019). Eles verificaram que somente os índices Ca4227, G4300, $\mathrm{CN}_{1}, \mathrm{CN}_{2}$ and $\mathrm{NaD}$ são apreciadamente afetados pelas abundâncias das anticorrelações, de modo que outros índices são em grande parte insensíveis à hipótese química imposta para a segunda geração de estrelas. Contudo, o He enriquecido da segunda geração afeta os índices sensíveis ao cálculo de idade (linhas de Balmer) devido à mudança da temperatura de Turf-Off (assumindo a mesma história de perda de massa e morfologia do HB de ambas as gerações). Assim, também foi confirmada a sensibilidade dos índices $\mathrm{H} \beta, \mathrm{H} \delta_{F}$ e $\mathrm{H} \gamma_{F}$ em relação à variação na fração de massa $\mathrm{Y}=$ 0,256 .

A Figura 1.11 (retirada de Coelho et al., 2012) mostra o espectro integrado sintético relativo às duas hipóteses químicas (i.e., SSPs) em comparação ao espectro observado para NGC 0104. Como entre as duas misturas foram variadas as abundâncias de C e N, a região 
sensível à molécula de CN apresenta os espectros bem distintos entre si. Em Coelho et al. (2011, 2012), o estudo foi realizado considerando somente uma única metalicidade: a típica de um aglomerado como 47 Tuc (NGC 0104) com $[\mathrm{Fe} / \mathrm{H}]=-0,7$. As conclusões do trabalho não podem então ser facilmente extendidas para a vasta maioria dos AGs.

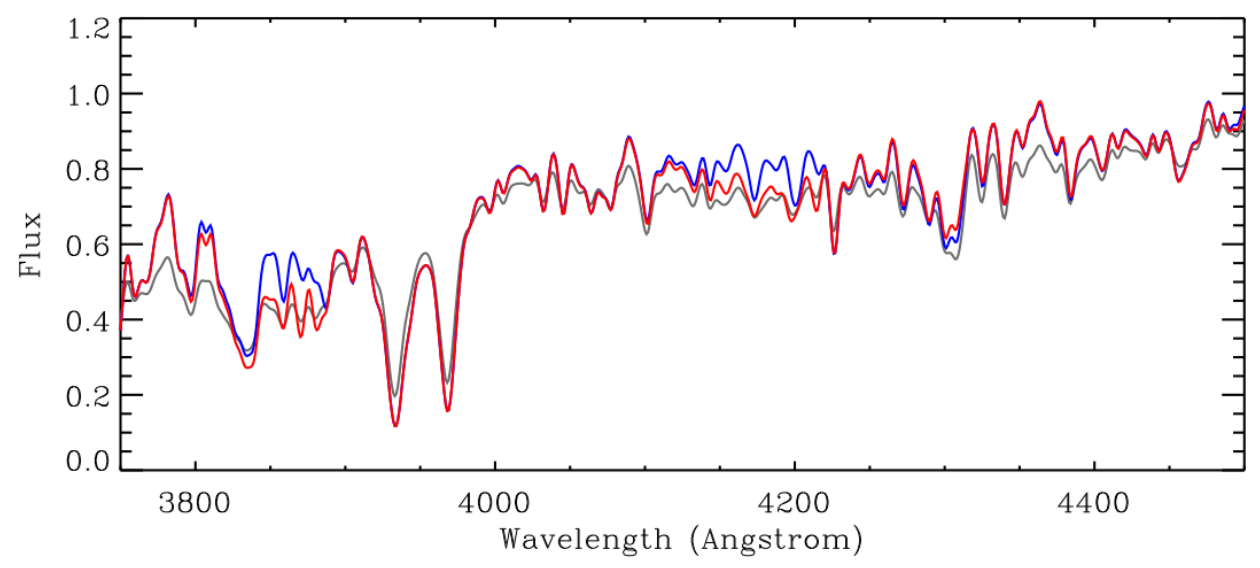

Figura 1.11: Espectros integrados sintéticos calculados com os modelos de mistura padrão (azul) e com as variações químicas CNONa e Y enriquecido (vermelho), na região espectral sensível à molécula $\mathrm{CN}$. $\mathrm{O}$ espectro observado de NGC 0104 é dado em cinza. Crédito: Coelho et al. (2012).

O principal objetivo de presente trabalho é expandir os resultados de Coelho et al. (2011) para outros regimes de metalicidade. Para tal, selecionamos outros dois AGs, um menos e outro mais metálico em relação à NGC 0104, como estudos de caso. Além disso, reavaliamos o processo de síntese espectral estelar, analisando listas de opacidade atômica e molecular na literatura, além de aumentarmos a cobertura em comprimento de onda do estudo no infravermelho próximo, de 6000 para $9300 \AA$.

Deste modo, no Capítulo 2 apresentamos os dados observacionais usados como referências do nosso trabalho. Em "Metodologia", Capítulo 3, elencamos as ferramentas e processos para a modelagem de atmosfera e síntese espectral estelar usados e opacidades moleculares e atômicas adotadas. A grade de espectros sintéticos é apresentada no Capítulo 4 e sua aplicação é apresentada no Capítulo 5. Nos Capítulos 6 e 7 são discutidas as conclusões e perspectivas do trabalho, respectivamente, e algumas informações adicionais são dadas no Apêndice. 
Capítulo 2

\section{Dados Observacionais}

Nas seções seguintes, apresentamos as informações relativas aos dados observacionais considerados no trabalho. Utilizamos espectros observados do Sol, Arcturus e de linhas telúricas (Seções 2.1 e 2.2 para calibrar o processo de síntese espectral, a ser discutido no Capítulo 3. Estas estrelas foram escolhidas devido à alta acurácia de seus parâmetros atmosféricos e alta resolução espectral. Isto nos permite avaliar em grande detalhe o quão bem nossos espectros sintéticos estão representando as observações e, assim, fazer melhores escolhas entre diferentes padrões de abundância e linhas de opacidade atômica e moleculares, disponíveis na literatura.

Para testar a aplicabilidade do trabalho em diferentes regimes de metalicidade (Capítulos 4 e 5), na Seção 2.3 apresentamos os dados observacionais dos aglomerados globulares que usamos na aplicação de nossos espectros sintéticos.

\subsection{Estrelas de referência: Sol e Arcturus}

O dado observacional do Sol é do Atlas de Fluxo de Kurucz (2005b), que compreende o intervalo de 300 a $1000 \mathrm{~nm}$, resolução espectral gaussiana $R_{\mathrm{LSF}}=300000$ e razão sinalruído $(\mathrm{S} / \mathrm{N})$ de 2600 a 9000 (a depender do intervalo de comprimento de onda). Já o dado observacional de Arcturus é de Hinkle et al. (2000) e compreende o intervalo de 372 a $930 \mathrm{~nm}$, com resolução espectral gaussiana $R_{\mathrm{LSF}}=150000$ e S/N de aproximadamente 1000. Imagens reais do Sol e Arcturus são apresentadas na Figura 2.1 e seus espectros observados normalizados na Figura 2.2, em regiões centradas nas linhas de Ca II [H-K] (Ca II), Tripleto de $\mathrm{Mg}(\mathrm{Mg} T)$ e Dubleto de $\mathrm{Na}(\mathrm{Na} D)$. 

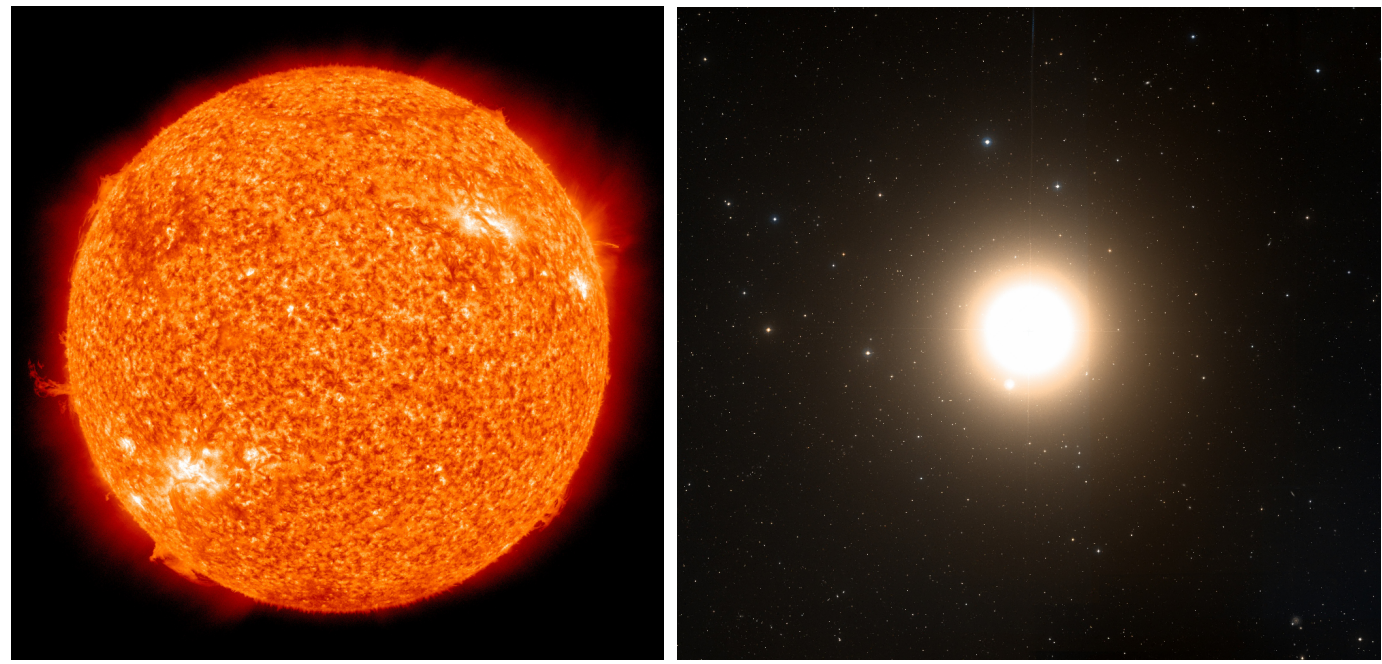

Figura 2.1: Estrelas: Sol (NASA, 2010) à esquerda e Arcturus à direita (Lasker et al., 1996).
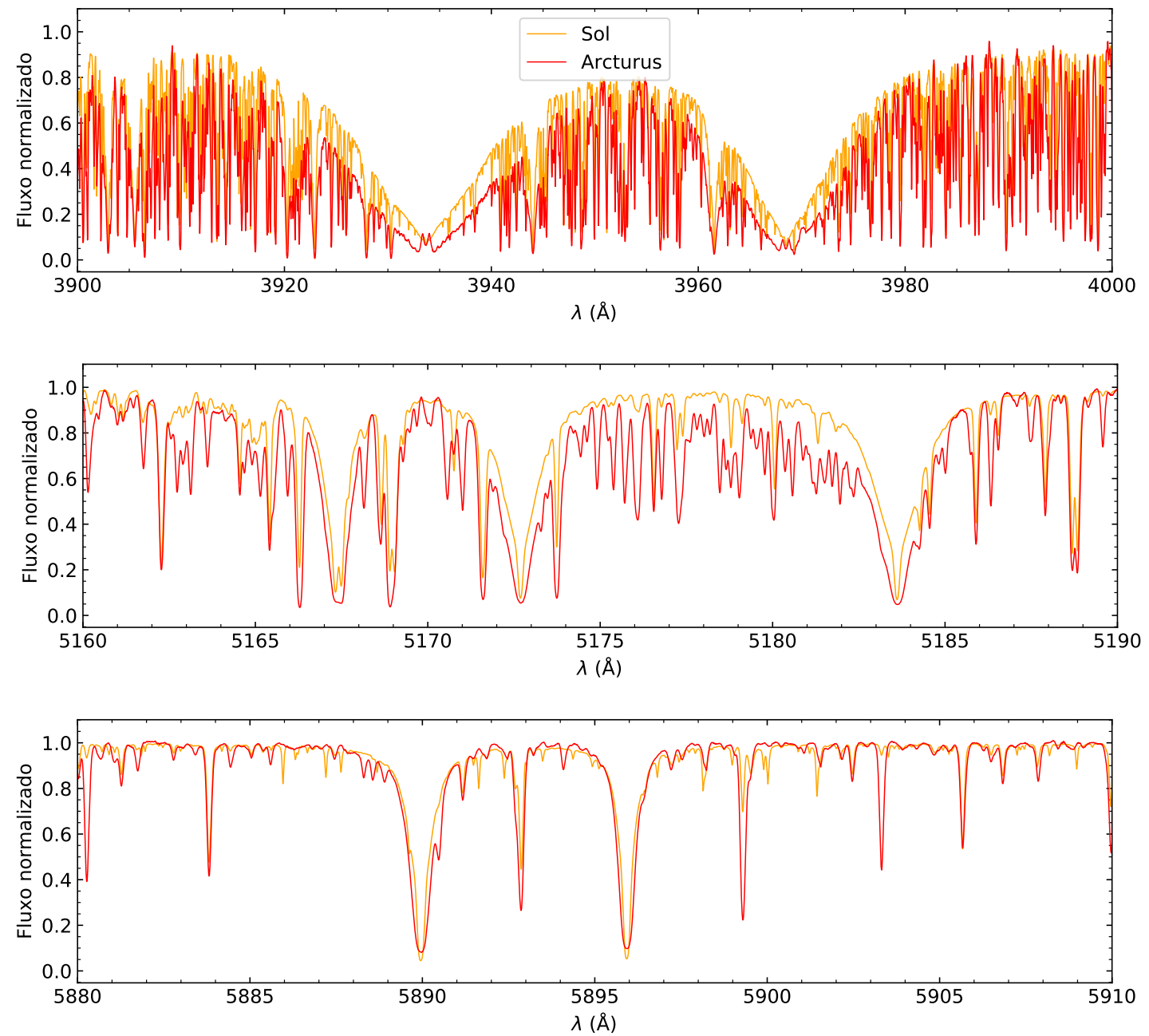

Figura 2.2: Espectros de fluxo normalizado observados do Sol (laranja) e Arcturus (vermelho) na região do Ca II (cima), $\operatorname{Mg} T$ (meio) e $\mathrm{Na} D$ (baixo). 


\subsection{Atlas de linhas telúricas}

No intervalo de comprimento de onde que estamos interessados, há regiões com forte contaminação telúrica (Hinkle et al., 2000) como ilustrado na Figura 2.3, da mesma observação relativa à Arcturus. Como os espectros sintéticos não levam em conta a influência telúrica, determinamos quais regiões de comprimento de onda poderiam ser utilizadas nas análises de listas de opacidade. A determinação destas regiões depende fundamentalmente de quanta influência telúrica é tolerada. Ou, de outro modo, a partir de qual valor de fluxo normalizado das linhas telúricas ignora-se o elemento de comprimento de onda para análise. Os detalhes deste estudo são discutidos na Seção 3.4, sobre a calibração da síntese espectral.

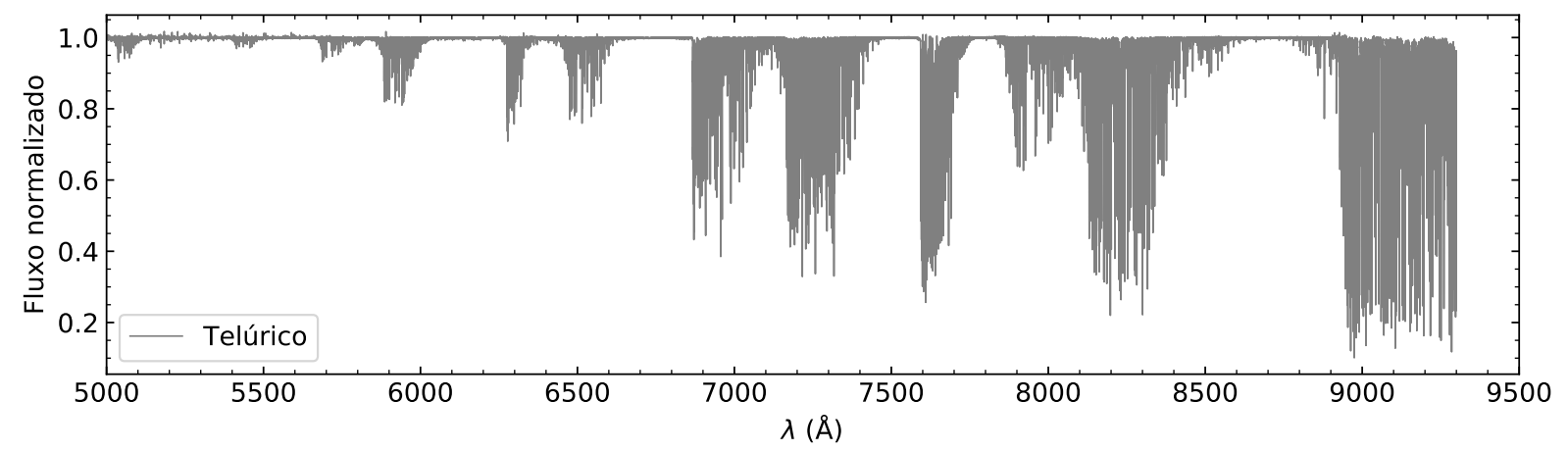

Figura 2.3: Espectro de fluxo normalizado das linhas Telúricas (cinza).

\subsection{Aglomerados globulares}

Para testarmos nosso trabalho, devemos confrontar nossos resultados com dados observacionais disponíveis na literatura. Como baseamos esta pesquisa no estudo de Coelho et al. (2011), temos NGC 0104 como o AG de "controle", isto é, nosso objeto de referência com o qual avaliamos nossos primeiros resultados. Por extensão, buscamos outros dois aglomerados que representam sistemas de baixa e alta metalicidade, em comparação ao AG controle.

Partimos do trabalho de Martins et al. (2019), que avalia a performance de bibliotecas de espectros estelares na reprodução de espectros integrados de AGs. Este estudo fez uso 
dos espectros integrados observados de Schiavon et al. (2005) (daqui em diante "Schiavon" quando aplicável), obtidas com o telescópio de 4m, Blanco, no Cerro Tololo Interamerican Observatory. Os espectros cobrem o intervalo de comprimento de onda 3600 a $6300 \AA$, com resolução FWHM 3, 1 A (e.g., Figura 2.4, referente a NGC 0104). Schiavon et al. (2005) priorizaram AGs que tivessem CMDs de alta qualidade disponíveis na literatura, de modo a calibrar parâmetros como idade, metalicidade e a morfologia do HB de forma confiável.

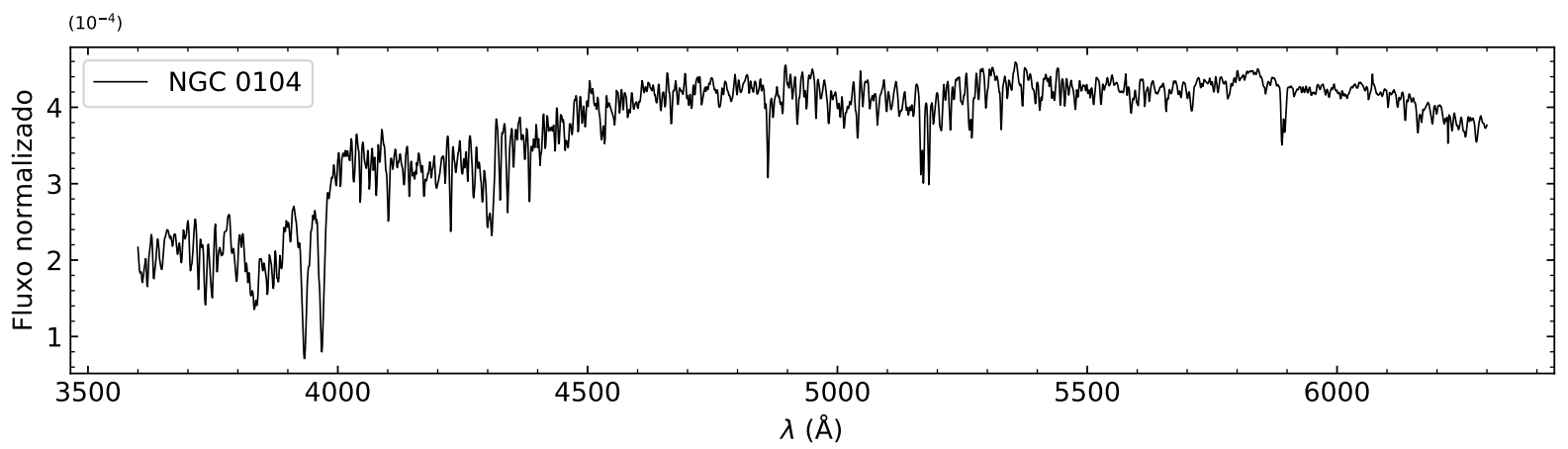

Figura 2.4: Espectro integrado de NGC 0104, observado por Schiavon et al. (2005), de 3600 a $6300 \AA$.

Em Martins et al. (2019), espectros integrados sintéticos de AGs foram modelados a partir de CMDs disponíveis na literatura, de tal modo que os espectros individuais das estrelas são somados, ponderados pela respectiva magnitude absoluta num dado CMD, para cada AG. Tivemos acesso aos espectros integrados sintéticos e aos CMDs convertidos em $T_{\text {eff }}$ vs $\log g$ calculados em Martins et al. (2019, comunicação privada).

Os espectros integrados sintéticos dos AGs foram calculados a partir de bibliotecas estelares empíricas e teóricas, com a de Coelho (2014) entre elas, a qual tivemos acesso. Deste modo, podemos verificar nosso trabalho também em relação aos espectros integrados calculados a partir de uma biblioteca teórica disponível na literatura. Da lista de AGs de Martins et al. (2019), selecionamos os AGs NGC 1904 e NGC 5927 como representantes de sistemas menos e mais metálicos, respectivamente, em relação ao nosso AG de referência, NGC 0104, conforme relaciona a Tabela 2.1.

\footnotetext{
1 http://specmodels.iag.usp.br. P. Coelho e colaboradores.
} 
Tabela 2.1 - Aglomerados globulares usados nesse trabalho e respectivas metalicidades como publicadas em Harris (1996) - 10a edição.

\begin{tabular}{|c|c|}
\hline NGC & {$[\mathbf{F e} / \mathbf{H}]$} \\
\hline 1904 & $-1,60$ \\
\hline 0104 & $-0,72$ \\
\hline 5927 & $-0,49$ \\
\hline
\end{tabular}

Buscando por referências mais atuais, encontramos o projeto WAGGS (Usher et al., 2017) (do inglês, the WiFeS Atlas of Galactic Globular cluster Spectra), que consiste de uma biblioteca de mais de 86 espectros integrados de AGs da Via Láctea (VL) e do Grupo Local (GL). Os espectros cobrem o intervalo de 3270 a $9050 \AA$ de comprimento de onda, com resolução espectral $R \sim 6800$, observados com o Wide-Field Spectrograph (WiFeS) no telescópio de 2,3 metros da Australian National University (ANU). A amostra compreende o intervalo de $-2.4 \leq[\mathrm{Fe} / \mathrm{H}] \leq-0.1$ de metalicidades de AGs da VL e inclui AGs desde alguns kpc do centro Galáctico (NGC6528) até aproximadamente 40 kpc no halo (NGC 7006), e outros 22 AGs em galáxias satélites da VL.

Cada observação foi feita com duas configurações de grade, dividindo a imagem em regiões vermelha e azul do espectrógrafo. Em uma das configurações, a luz foi dividida entre as grades U7000 e R7000 que cobrem, respectivamente, os invervalos de 3270 a $4350 \AA$ $(0,27 \AA / p x)$ e 5280 a $7020 \AA(0,44 \AA / p x)$. Na outra, foram usadas as grades B7000 e I7000, cobrindo os invervalos 4170 a $5540 \AA(0,37 \AA /$ px $)$ e 6800 a $9050 \AA(0,57 \AA /$ px $)$, respectivamente. Conforme descrito, há superposição entre as grades U7000 com B7000, B7000 com R7000, e R7000 com I7000. A Figura 2.5 ilustra o espectro observado de NGC 0104, evidenciando as regiões de cada grade, bem como as regiões de superposição entre elas.

Os espectros do projeto WAGGS, diferentemente dos espectros de Schiavon, tiveram que ser tratados de modo a cada AG ter um único espectro (e não quatro, em obervações superpostas, conforme descrito no parágrafo anterior). Para juntar os espectros de cada grade do WAGGS priorizamos a grade com maior S/N. Usamos o módulo PyAsl do pacote PyAstronomy (Czesla et al., 2019) para corrigir os espectros WAGGS para o sistema de 
referência em repouso. As velocidades radiais de cada espectro foram medidas pela Prof. Dra. Paula R. T. Coelho (comunicação privada e Gonçalves et al., 2020).

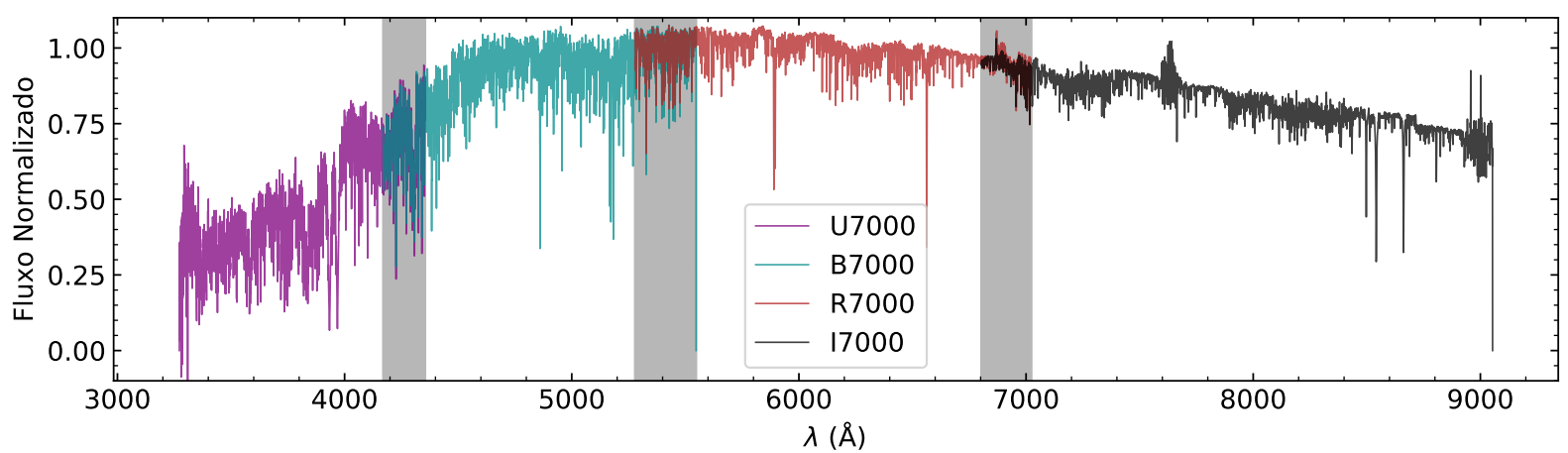

Figura 2.5: Espectro de fluxo observado de NGC0104 por Usher et al. (2017). As regiões em cinza representam os intervalos em que há superposição entre os espectros. Observações das grades U7000, B7000, R7000 e I7000 estão ilustrados em roxo, ciano, vermelho escuro e preto, respectivamente.

A Figura 2.6 ilustra a posição de diversos aglomerados globulares Galácticos, com a imagem do centro da Via Láctea (Mellinger, 2009) centralizada na figura. Representamos no mapa do céu de 2.6, em vermelho, o catálogo de Francis e Anderson (2014) que conta com 157 AGs, usados para estimar a distância ao centro Galáctico, baseado na 10ª edição do catálogo de Harris (1996). Em amarelo estão indicados alguns dos aglomerados globulares da VL observados por Usher et al. (2017), escolhidos arbitrariamente, e em ciano são os aglomerados do nosso trabalho.

De modo a consolidar os dados observacionais, mostramos na Figura 2.7 três paineis de espectros observados na coluna à esquerda e diagramas Hestzprung-Russel (diagramas-HR, DHR) na coluna à direita, com a barra de cor $M_{V}$ representando a magnitude absoluta na banda $\mathrm{V}$ das estrelas. Cada linha da figura é relativa a um aglomerado diferente e, do painel superior ou inferior, estão organizados do menos ao mais metálico. Para comparar os dois espectros observados, foi feita a normalização pela integral do fluxo de cada espectro, no intervalo de comprimento de onda de 3600 a $6300 \AA$, que corresponde aos espectros de Schiavon por estarem contidos no intervalo dos espectros observados pelo WAGGS. 


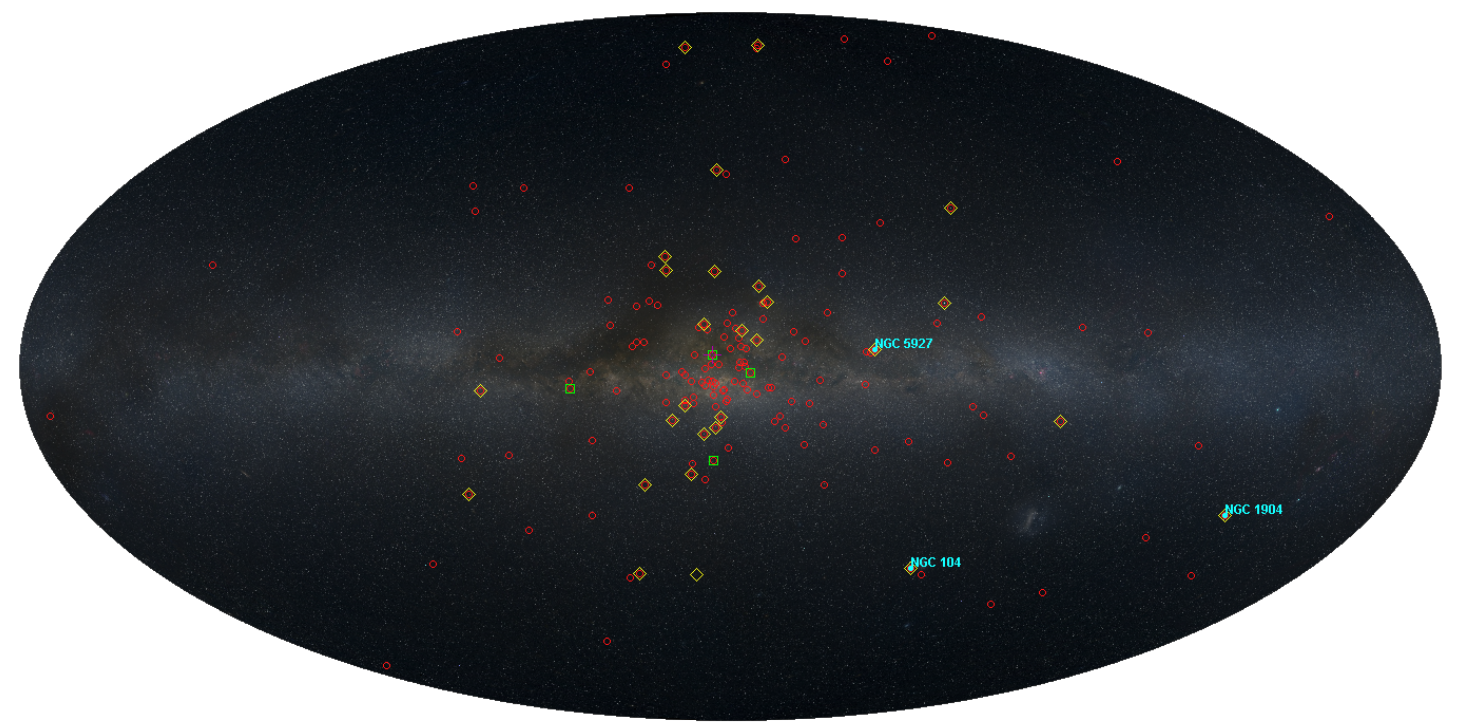

Figura 2.6: Representação de aglomerados globulares da Via Láctea. Em vermelho, aglomerados do catálogo de Francis e Anderson (2014); em amarelo, alguns aglomerados de Usher et al. (2017); e em ciano, os aglomerados adotados para este trabalho. Esta imagem foi produzida com o software Aladin Sky Atlas (Bonnarel et al., 2000).
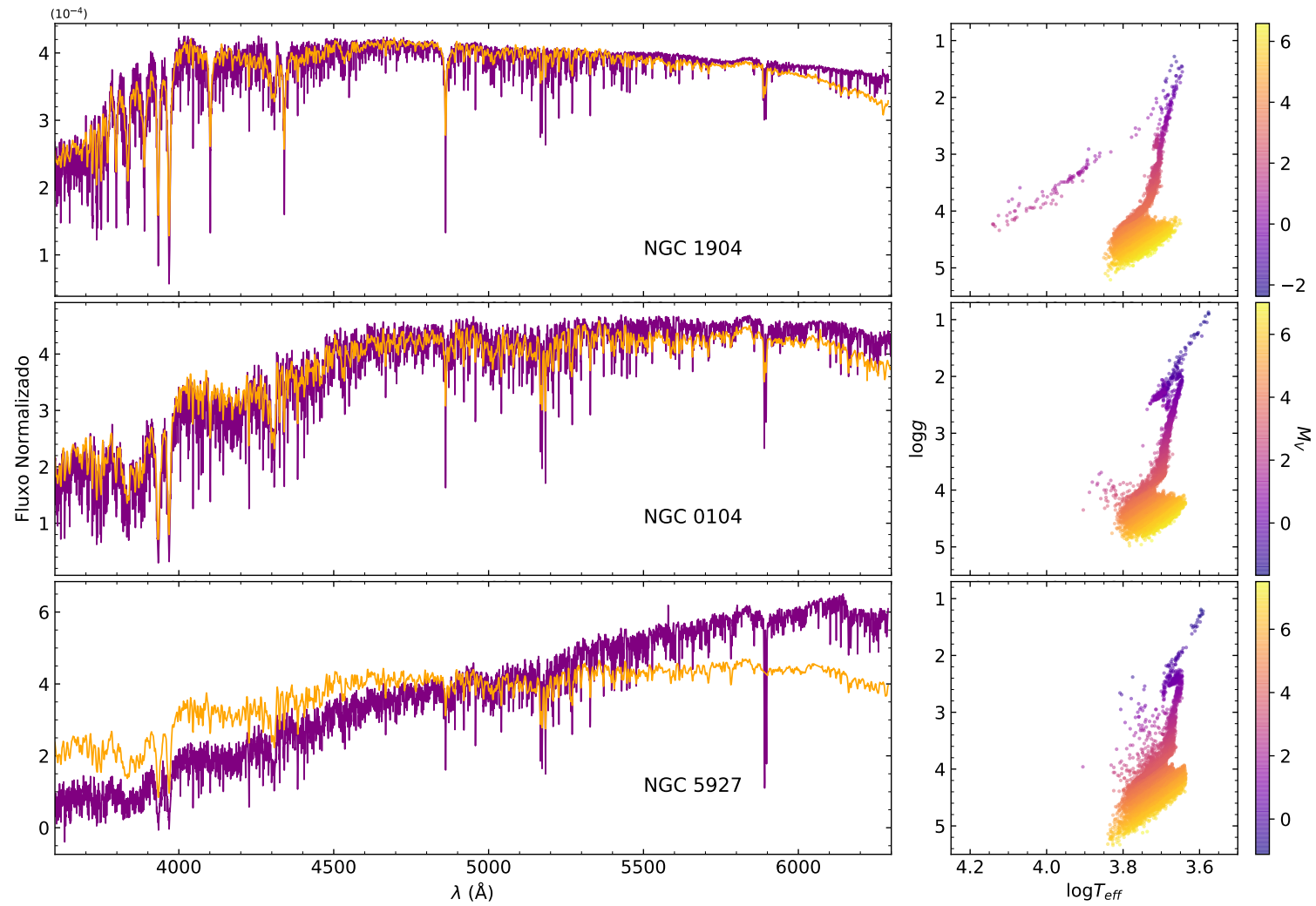

Figura 2.7: Painel de espectros observados (esquerda) de NGC 1904, NGC 0104 e NGC 5927 (de cima para baixo) do WAGGS (roxo) e Schiavon (laranja), de 3600 a $6300 \AA$, e seus respectivos diagramas-HR (direita). 
Ainda da Figura 2.7, os parâmetros dos diagramas-HR apresentados, temperatura efetiva $T_{\text {eff }}$, gravidade superficial $\log g$ (eixos $x$ e $y$, respectivamente) e magnitude absoluta visível $M_{V}$ (barra de cor) foram inferidos por Martins et al. (2019), com base nos dados observados de Piotto et al. (2002). Assim, para cada aglomerado, ilustramos a contribuição da luz de cada estrela pela sua magnitude absoluta, sendo mais brilhante quanto menor o valor de sua $M_{V}$ (ou quanto mais para o roxo estiver), e o espectro integrado de cada AG.

Nota-se na Figura 2.7 uma grande diferença entre os contínuos das observações de NGC 5927. Schiavon et al. (2005) aponta em seu artigo que houve dificuldade em traçar o aglomerado para a observação. Dr. Christopher Usher, por comunicação privada, nos informou da presença de uma estrela de brilho variável de longo período (LPV, do inglês, Long-period variable), que contribui com $25 \%$ do fluxo na banda I do espectro em NGC 5927. As variações estocásticas no brilho de estrelas gigantes são responsáveis pela maior fonte de incerteza dos espectros integrados do WAGGS (veja Figura 15 de Usher et al. (2017) e Seção 3.3 do mesmo artigo).

Para concluir esta seção de apresentação dos aglomerados escolhidos, mostramos imagens reais com cores compostas de NGC 1904, NGC 0104 e NGC 5927, na Figura 2.8. De (a) a (c), do AG menos ao mais metálico, respectivamente, se pode ter uma breve ideia da complexidade (e da beleza) que são estes sistemas de estrelas. 


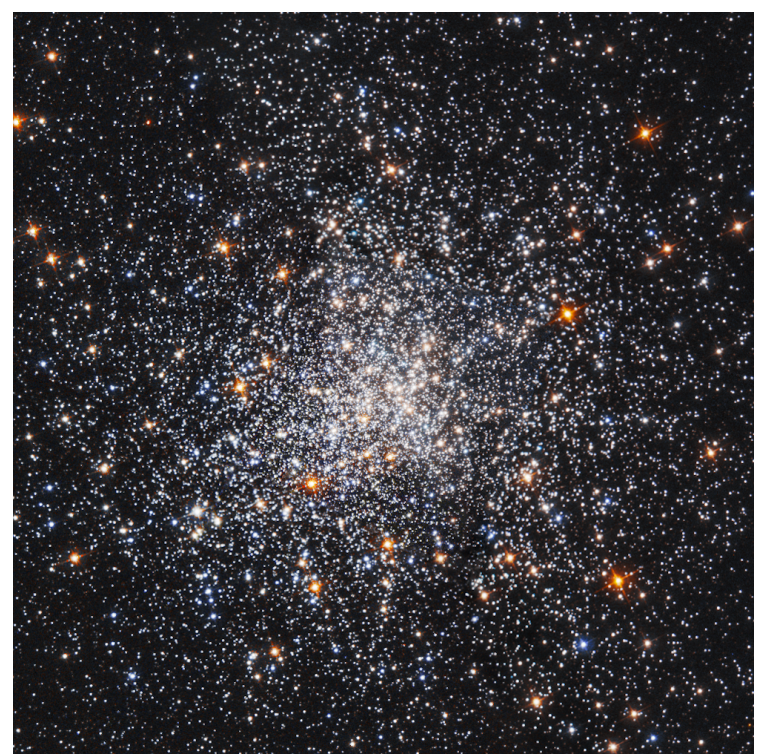

(a) NGC 1904

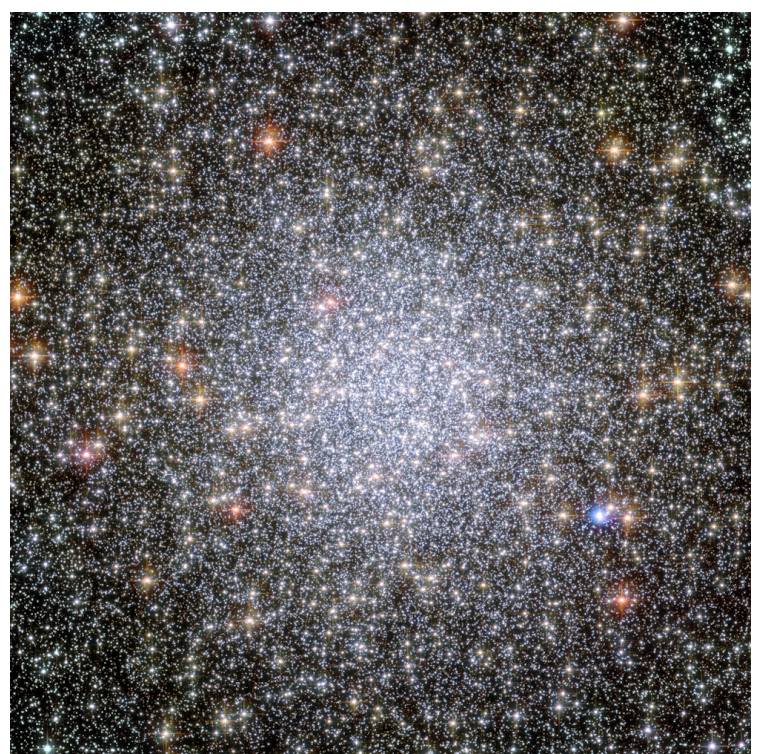

(b) NGC 0104

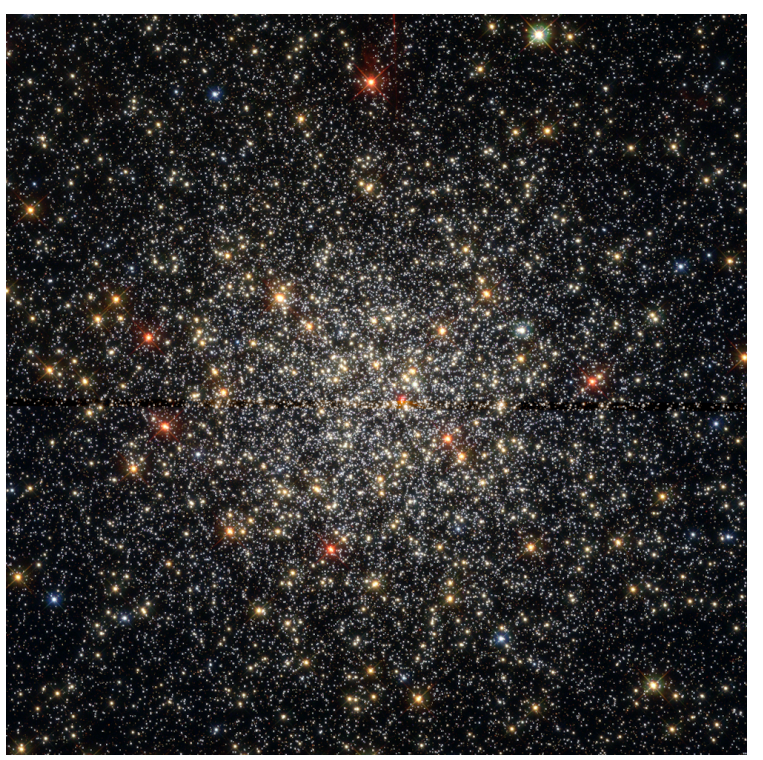

(c) NGC 5927

Figura 2.8: Imagens reais dos aglomerados globulares: (a) NGC 1904 (Messier 79) (NASA/STScI, 2017); (b) NGC 0104 (47 Tucanae) (NASA/ESA/STScI/AURA/ESA-HST, 2015); (c) NGC 5927 (Hubble Legacy Archive, 2016). 
Capítulo 3

\section{Metodologia}

Antes de abordarmos os espectros integrados de aglomerados globulares, precisamos construir espectros estelares sintéticos. Para esta tarefa, existem duas etapas fundamentais: a modelagem da atmosfera e a síntese espectral estelar (Seções 3.1 e 3.2, respectivamente). O primeiro fornece os ingredientes necessários para que o segundo calcule o produto final: o espectro estelar teórico (i.e., sintético). Como nosso objetivo é construir uma grade de espectros para o estudo de aglomerados globulares, desenvolvemos um programa para nos auxiliar, o PyGLoBsterS, apresentado na Seção 3.3 .

Para alguns dos arquivos de entrada da síntese espectral, como as opacidades atômicas e moleculares, há diferentes opções disponíveis na literatura. Com isto, tivemos que escolher o padrão de abundâncias solar e as listas de opacidade mais adequadas para calcular os espectros sintéticos, como descrito na Seção 3.4. Para tanto, usamos o Sol para calibrar e Arcturus para testar nosso processo de síntese espectral.

\subsection{Modelos de atmosfera estelar}

Dado um conjunto de parâmetros atmosféricos de entrada (como temperatura efetiva, gravidade superficial, velocidade de microturbulência e abundâncias químicas), modelos de atmosfera estelar descrevem as propriedades termodinâmicas da atmosfera da estrela em função de algum parâmetro de profundidade (e.g., profundidade ótica ou coluna de massa). Estes modelos, por sua vez, são arquivos de entrada em códigos de síntese espectral. Desta forma, variando os parâmetros de entrada dos modelos, podemos calcular es- 
pectros sintéticos estelares com diversas propriedades de $T_{\text {eff }}, \log g$, abundâncias químicas, entre outros parâmetros.

Algumas hipóteses devem ser consideradas no cálculo dos modelos, como se há o equílibrio local termodinâmico (LTE, do inglês, Local Thermodynamic Equilibrium), se o sistema de coordenadas é esférico ou plano paralelo, qual a teoria de comprimento de mistura (do inglês, Mixing Length Theory) e o parâmetro de "overshooting" referente à convecção da estrela. Na literatura encotramos diversos programas que calculam modelos de atmosfera estelar, por exemplo: ATLAS (Kurucz, 1970, 1993; Sbordone et al., 2004; Sbordone, 2005), TLUSTY (Hubeny, 1988), MARCS (Gustafsson, 1971), PHOENIX (Hauschildt, 1992, 1993; Hauschildt e Baron, 1995; Hauschildt et al., 1995; Allard e Hauschildt, 1995, Hauschildt et al., 1996; Baron et al., 1996), e PoWR (Hamann e Schmutz, 1987; Gräfener et al., 2002; Hamann e Gräfener, 2003, Sander et al., 2015), entre outros.

Para calcular um modelo de atmosfera estelar são utilizadas duas principais abordagens estatísticas: ODF (do inglês, Opacity Distribution Function) e OS (do inglês, Opacity Sampling). Em relação à ODF, o método OS possui a vantagem de determinar as opacidades com a modelagem de cada atmosfera, o que permite mais flexibilidade em explorar diversos padrões de abundância química (veja, Hauschildt et al., 2001; Kurucz, 2005a, para mais detalhes). Esta diferença é importante para a aplicação deste trabalho nos espectros integrados de Aglomerados Globulares, conforme discussão sobre os padrões de abundância CNONa na Seção 1.5. Por consistência, para compararmos nossos resultados com os de Coelho et al. (2011), que motivou esta pesquisa, utilizamos o programa ATLAS12 $1 \mathrm{Wu}$ rucz, 2005a) que calcula modelos de atmosfera estelar pelo método OS, com geometria plano-paralela (1-dimensão), sob o regime LTE.

Neste trabalho, consideramos três padrões de abundâncias para o Sol: Grevesse e Sauval (1998); Asplund et al. (2005, 2009). Para os dois primeiros padrões, modelos de atmosfera pré-calculados foram adotados do website de F. Castell:3. O modelo solar com abundâncias

\footnotetext{
1 http://kurucz.harvard.edu/programs/atlas12/

2 http://atmos.obspm.fr/index.php/download

3 http://wwwuser.oats.inaf.it/castelli/sun.html
} 
de Asplund et al. (2009) foi calculado para essa dissertação com o ATLAS12. Para Arcturus, calculamos um modelo de atmosfera estelar com as abundâncias químicas e parâmetros atmosféricos dados por Ramírez e Prieto (2011). A Tabela 3.1 apresenta os principais parâmetros atmosféricos adotados para cada estrela.

Tabela 3.1 - Parâmetros atmosféricos e modelos de atmosfera para o Sol e Arcturus. Refira-se à última coluna da tabela para as abundâncias químicas de cada modelo no formato do ATLAS12, com as respectivas seções no Apêndice.

\begin{tabular}{|c|c|c|c|c|c|c|}
\hline & $\begin{array}{l}T_{\text {eff }} \\
(\mathbf{K})\end{array}$ & $\log g$ & $\begin{array}{c}V_{\text {turb }} \\
(\mathrm{km} / \mathrm{s})\end{array}$ & {$[\mathbf{F e} / \mathbf{H}]$} & Modelo de Atmosfera & Abundâncias \\
\hline Sol & 5777 & 4,4377 & 1,0 & 0,0 & $\begin{array}{l}\text { Grevesse e Sauval }(1998) \\
\text { Asplund et al. } \\
\text { Asplund et al. } \\
\end{array}$ & \begin{tabular}{|c|} 
A.1.1 \\
A.1.2 \\
A.1.3 \\
\end{tabular} \\
\hline Arcturus & 4282 & 1,66 & 1,74 & $-0,54$ & Ramírez e Prieto (2011) & A.2.1 \\
\hline
\end{tabular}

\subsection{Síntese espectral}

A síntese de espectros estelares é um dos métodos usados para estudar estrelas e populações estelares. Quando confrontamos espectros sintéticos com espectros observados de média/alta resolução, podemos extrair informações estelares fundamentais como parâmetros atmosféricos e composição química (e.g., Barbuy, 1994; Meléndez et al., 2010; Meléndez, 2014; Dias et al., 2016; Tsantaki et al., 2018; Queiroz et al., 2020).

Existe diferentes programas para o cálculo de espectros estelares de alta resolução, por exemplo, PFANT (Spite, 1967; Barbuy et al., 2018), SPECTRUM (Gray, 1999), NESSY (Tagirov et al., 2017), SYNSPEC (Hubeny e Lanz, 2011) e SYNTHE (Kurucz e Avrett, 1981; Kurucz e Furenlid, 1979; Sbordone et al., 2004; Sbordone, 2005), entre outros. Apesar das características de cada programa serem um pouco diferentes (por exemplo, adotar a hipótese LTE ou não, serem multiplataforma ou não, outputs personalizados, entre outros detalhes), todos dependem de um modelo de atmosfera estelar e de listas de transições atômicas e moleculares. 
Optamos por utilizar o SYNTHE 4 , por consistência ao trabalho de Coelho et al. (2011). Este programa de síntese espectral consiste em um conjunto de aplicações em FORTRAN, desenhados para produzir espectros tanto de baixa quanto alta resolução espectral, para ser comparado com espectros de intensidade ou fluxo das estrelas.

\subsection{PyGlobsterS}

PyGlobsterS (PYthon GLOBular cluSTER Synthesizer) ${ }^{5}$ é um conjunto de scripts em Python 3.x para GNU-LINUX, desenvolvidos neste projeto de mestrado com o intuito de automatizar os cálculos de modelos atmosféricos e sínteses espectrais estelares, usando ATLAS12 e SYNTHE, respectivamente, baseado nas documentações Atlas Cookbook ${ }^{6}$ (Sbordone e Bonifacio, 2005) e Working Notes 7 (Jauregi, 2005).

Este pacote conta com cinco módulos que podem ser usados para calcular modelos de atmosfera (PyATLAS, Subseção 3.3.1), espectros sintéticos (PySYnTHE, Subseção 3.3.2), alterar dispersão e resolução espectral para comparar com espectros de referência (e.g., Sol e Arcturus) e calcular a performance dos resultados através de abordagens estatísticas. Especificamente para este trabalho, criamos também um módulo para produzir listas de linhas de opacidade atômicas com base em outras disponíveis na literatura (conforme discussão na Seção 3.4.

Além dos módulos acima, um conjunto de scripts em Python e Shell são disponibilizados para o tratamento de dados como, por exemplo, teste de convergência dos modelos de atmosfera calculados, convolução espectral, correção de velocidade radial de espectros observados de aglomerados globulares, cálculo de espectro integrado com base em uma distribuição de estrelas no DHR, cálculo de espectro sintético somente com linhas de opacidade atômica ou molecular (à escolha do usuário), cálculo de índices de Lick, criação de gráficos, entre outros. Muitos destes processos serão oportunamente discutidos nas subseções e capítulos adiante e mais detalhes sobre o algoritmo do PyGLOBSTERS serão devidamente documentados e publicados no futuro.

\footnotetext{
4 http://wwwuser.oats.inaf.it/castelli/sources/synthe.html

5 https://github.com/ViniciusBranco/PyGlobsterS

6 http://atmos.obspm.fr/index.php/documentation/7

7 http://atmos.obspm.fr/images/stories/download/working_notes.pdf
} 
Na Figura 3.1, apresentamos uma visão geral do fluxograma do pacote que permeia todo o desenvolvimento deste projeto. Os módulos são executados a partir do "PyGlobsterS.py", com um único comando no terminal 8 . As próximas subseções apresentam os módulos PyATLAS e PySYNTHE, respectivamente. Os módulos REBIN, ADEV e GF, bem como outras tarefas que compõem o pacote PyGlobsters, são discutidos ao longo do texto quando necessário.

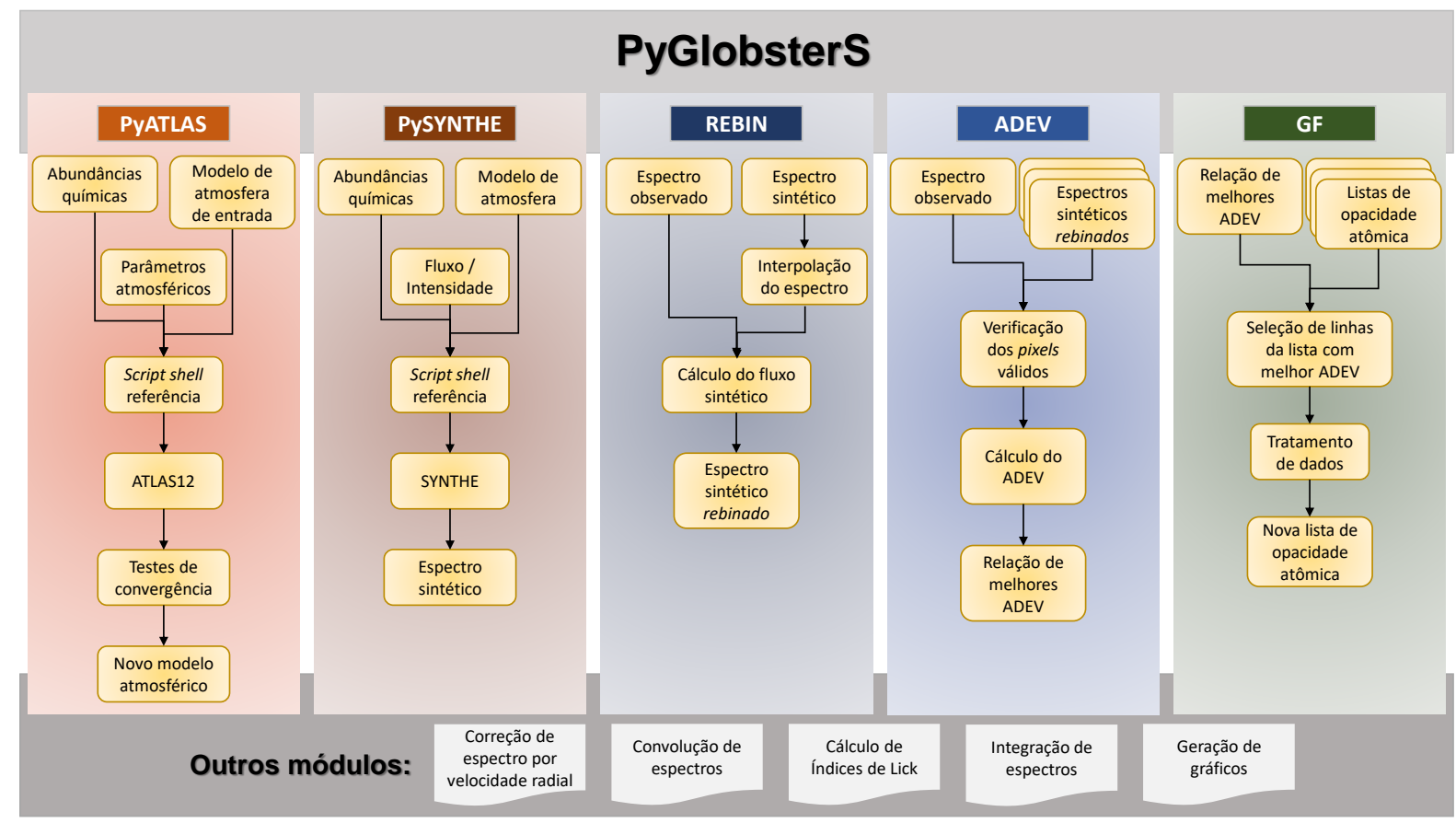

Figura 3.1: Fluxograma do pacote PyGlobsterS. As áreas coloridas representam o escopo de cada módulo. As caixas amarelas são os principais processos ou informações necessários. A flecha preta indica o fluxo do processo. As caixas em cinza claro, no rodapé do fluxograma, são outras tarefas que podem ser realizadas com os scripts disponíveis no PyGLOBsterS.

\subsubsection{PyATLAS}

Para criar uma grade espectros sintéticos estelares, precisamos de um modelo de atmosfera para cada espectro. PyATLAS é um método do pacote PyGLOBSTERS que gerencia e executa automaticamente o ATLAS12, manipulando seus parâmetros de entrada e saída. Para o cálculo de um modelo de atmosfera com o PyATLAS é necessário informar alguns dos principais parâmetros de entrada do ATLAS12:

\footnotetext{
8 python3 pyglobsters.py $\mathrm{x} \times \mathrm{x} \times \mathrm{x}$, onde " $\mathrm{x}$ " é um valor booleano para executar os módulos, respectivamente, PyATLAS, PySYNTHE, REBIN, ADEV, GF.
} 
- Modelo de atmosfera de referência;

- Matriz de abundâncias química:9.

- Fator de escala de metalicidade;

- Número de iterações;

- Quantidade de camadas da estrela;

- Temperatura efetiva $\left(T_{\text {eff }}\right)$;

- Gravidade superficial $(\log g)$;

- Velocidade de microturbulência $\left(V_{\text {turb }}\right)$;

- Comprimento de mistura;

- Cálculo com ou sem "overshooting";

- Profundidade ótica de Rosseland ( $\left.\tau_{\text {Ross }}\right)$;

- Limites inferior e superior do critério de convergência em $\tau_{\text {Ross }}$.

O PyATLAS altera um script shell (exemplo, "atlas12.sh") para executar o ATLAS12 com estas informações de entrada. O modelo calculado tem sua convergência testada pelo script "IfConv.pl", escrito em PERL e disponível para download com o ATLAS12. Conforme a documentação Atlas Cookbook (Subseção 3.2), o teste consiste em avaliar o erro atribuído ao cálculo do fluxo em cada camada da atmosfera. O PyATLAS avalia o $\tau_{\text {Ross }}$ das camadas do modelo recém calculado que o script "IfConv.pl" acusa não convergir, sendo $\tau_{\text {Ross }_{k}}$ a profundidade ótica de Rossland da $k$-ésima camada do modelo de atmosfera estelar calculado pelo ATLAS12. Consideramos que o modelo é adequado para uso se todas as camadas dentro do intervalo $\log \tau_{\text {Ross }}=-5$ e $\log \tau_{\text {Ross }}=1$ convergiram, pois esse é o intervalo onde as linhas do visível à banda H se formam (Mészáros et al., 2012).

\footnotetext{
${ }^{9}$ Os valores das abundâncias químicas estão em uma escala diferente ao tradicionalmente descrito na literatura, de modo que o valor "A(Kurucz)" da abundância de um elemento "A" ( $\left.\log \epsilon_{\mathrm{A}}\right)$ no ATLAS12 é dado por A(Kurucz) = A(Tradicional) - 12,04, conforme Atlas Cookbook (Subseção 3.2 .
} 
Por fim, PyATLAS automaticamente dá nome ao arquivo de output no formato ASCII, com base em informações dadas pelo usuário previamente como identificação do objeto (se aglomerado globular ou estrela) $)^{10} T_{\text {eff }}, \log g,[\mathrm{Fe} / \mathrm{H}]$, enriquecimento em elementos- $\alpha$, fração em Hélio e tipo de mistura química. Assim, o modelo de atmosfera de uma estrela do aglomerado globular NGC 0104, $\operatorname{com} T_{\text {eff }}=5500, \log g=2,5,[\mathrm{Fe} / \mathrm{H}]=-0,768,[\alpha / \mathrm{Fe}]$ $=0,4, \mathrm{Y}=0,256$ e "Alpha" indicando enriquecimento químico padrão do aglomerado (sem as variações químicas CNONa, por exemplo), terá seu arquivo identificado como "NGC0104_t5500_g2.5_m0.768_p0.4_y0.256_Alpha.mod".

\subsubsection{PySYNTHE}

Assim como com o PyATLAS, para criar a nossa grade de espectros são necessárias várias execuções do programa de síntese espectral SYNTHE, cada execução com alguma modificação dos parâmetro de entrada. Neste sentido, PySYNTHE é um método que gerencia e executa automaticamente o SYNTHE, manipulando seus parâmetros de entrada e saída. Para o cálculo de um espectro estelar com o PySYNTHE é necessário informar alguns dos principais parâmetros de entrada do SYNTHE:

- Modelo de atmosfera;

- Abundâncias químicas;

- Lista de opacidades atômicas e moleculares;

- Comprimento de onda inicial e final (nm);

- Resolução Amostral ${ }^{11}\left(R_{\lambda}\right)$;

- Resolução espectral $\left(R_{\mathrm{LSF}}\right)$;

- Cálculo para o Fluxo ou Intensidade da estrela;

- Comprimentos de onda no Ar ou Vácuo.

\footnotetext{
${ }^{10}$ Para o caso de um aglomerado globular, os nomes dos arquivos de modelo são diferenciados pelos parâmetros atmosféricos das estrelas pertencentes ao aglomerado.

11 "Resolução amostral" é a resolução de cálculo, com passo em comprimento de onda $\Delta \lambda=\lambda_{2}-\lambda_{1}$, dada por $R_{\lambda}=\lambda / \Delta \lambda$.
} 
O PySYNTHE altera um script SHELL (exemplo, "synthe.sh") para executar o SYNTHE com estas informações. O espectro calculado está no formato ASCII e tem seu cabeçalho preparado para que outros scripts em PYTHON possam manipular. Seu nome de arquivo é alterado com informações dadas pelo usuário através dos parâmetros de entrada listados. Assim, um espectro sintético calculado de 300 a $1000 \mathrm{~nm}$ no ar, com a lista de linhas de opacidade atômica por R. Kurucz, com um modelo de atmosfera de uma estrela do aglomerado globular NGC 0104, com $T_{\text {eff }}=5500, \log g=2,5,[\mathrm{Fe} / \mathrm{H}]=-0,768,[\alpha / \mathrm{Fe}]=0,4$, $\mathrm{Y}=0,256$ e "Alpha" indicando enriquecimento químico padrão do aglomerado (sem as variações químicas CNONa, por exemplo), terá seu arquivo identificado como "NGC0104 t5500_ g2.5_ m0.768_ p0.4 y0.3_ Alpha_ 300_ 1000_ kurucz_ AIR.asc".

\subsection{Ingredientes da síntese espectral}

Essa seção trata da escolha de três parâmetros de entrada em particular: um padrão de abundância química solar a ser usado como referência, listas de opacidades moleculares e lista de opacidades atômica.

Iniciamos com a escolha do padrão de abundância solar (Seção 3.4.1). Em seguida explicamos as listas de opacidades moleculares adotadas (Subseção 3.4.2). Por fim, com base no estudo de três listas de opacidades atômicas disponíveis na literatura (Seção 3.4.3), criamos duas novas listas de linhas atômicas calibradas em nossas estrelas de referência (Seção 3.4.4).

\subsubsection{Abundância solar adotada}

Há diferentes determinações de abundâncias solares disponíveis na literatura (e.g., veja o review de Asplund et al. 2009). Dentre elas, calculamos espectros sintéticos com os padrões de Grevesse e Sauval (1998), Asplund et al. (2005) e Asplund et al. (2009).

A Figura 3.2 ilustra os espectros observado Kurucz, 2005b) (preto) e sintéticos do Sol, calculados com as diferentes hipóteses de abundância química solar: Grevesse e Sauval (1998) em azul, Asplund et al. (2005) em vermelho e Asplund et al. (2009) em verde. 
Calculamos espectros solares adotando o mesmo conjunto de listas de opacidades moleculares (conforme Subseção 3.4.2) e a lista de opacidades atômicas de Kurucz (2018). Todos os espectros, exceto o telúrico (Hinkle et al., 2000) (cinza claro), foram convoluídos para facilitar a visualização. Notamos que os espectros sintéticos representam bem o espectro observado do Sol na região azul do comprimento de onda até $\sim 6900 \AA$, em que a contaminação telúrica muito presente parece influenciar no espectro observado.

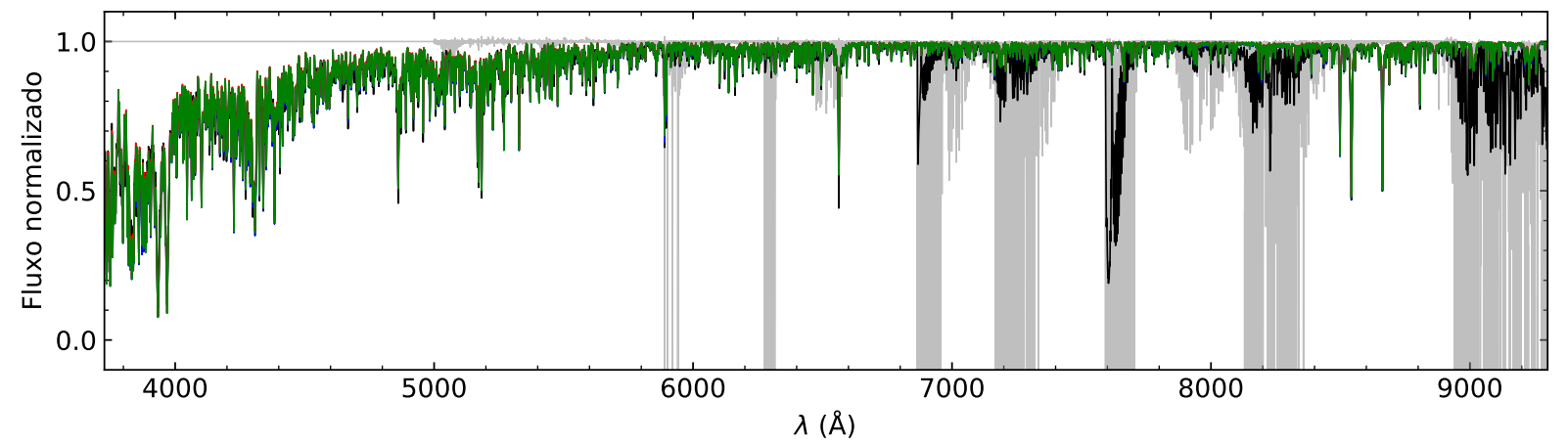

Figura 3.2: Espectros observado (Kurucz (2005b) em preto) e sintéticos do Sol (Grevesse e Sauval (1998) em azul, Asplund et al. (2005) em vermelho e Asplund et al. (2009) em verde) em comparação ao espectro telúrico (Hinkle et al., 2000), em cinza claro, de 3727 a 9300 Å.

Na Figura 3.3 escolhemos três regiões do espectro eletromagnético em que algumas linhas características são evidentes: Ca II [H-K] (cima, de 3920 a $3980 \AA$ A), MgT (meio, de 5160 a $5190 \AA$ ) e $\mathrm{Na} D$ (baixo, de 5880 a $5910 \AA$ ). Apesar de ser mais difícil verificar a diferença na profundidade das linhas entre os espectros no gráfico da região de Ca II [H-K], em $\operatorname{Mg} T$ e $\mathrm{Na} D$ é possível ver que as linhas do espectro sintético calculado com Grevesse e Sauval (1998) são as mais profundas, as do espectro sintético com Asplund et al. (2005) são as menos profundas, com Asplund et al. (2009) entre os dois. Essas diferenças são consistentes com as diferentes determinações de abundância solar, onde Grevesse e Sauval (1998) é o padrão mais rico em metais $(Z=0,00126)$, e Asplund et al. (2005) é o menos metálico $(Z=0,00083)$ dentre os padrões que estudamos $(Z=0,00094)$. Neste trabalho, demos preferência para as abundâncias de Asplund et al. (2009) por ser a mais recente (exceções são apontadas no texto quando necessário). 

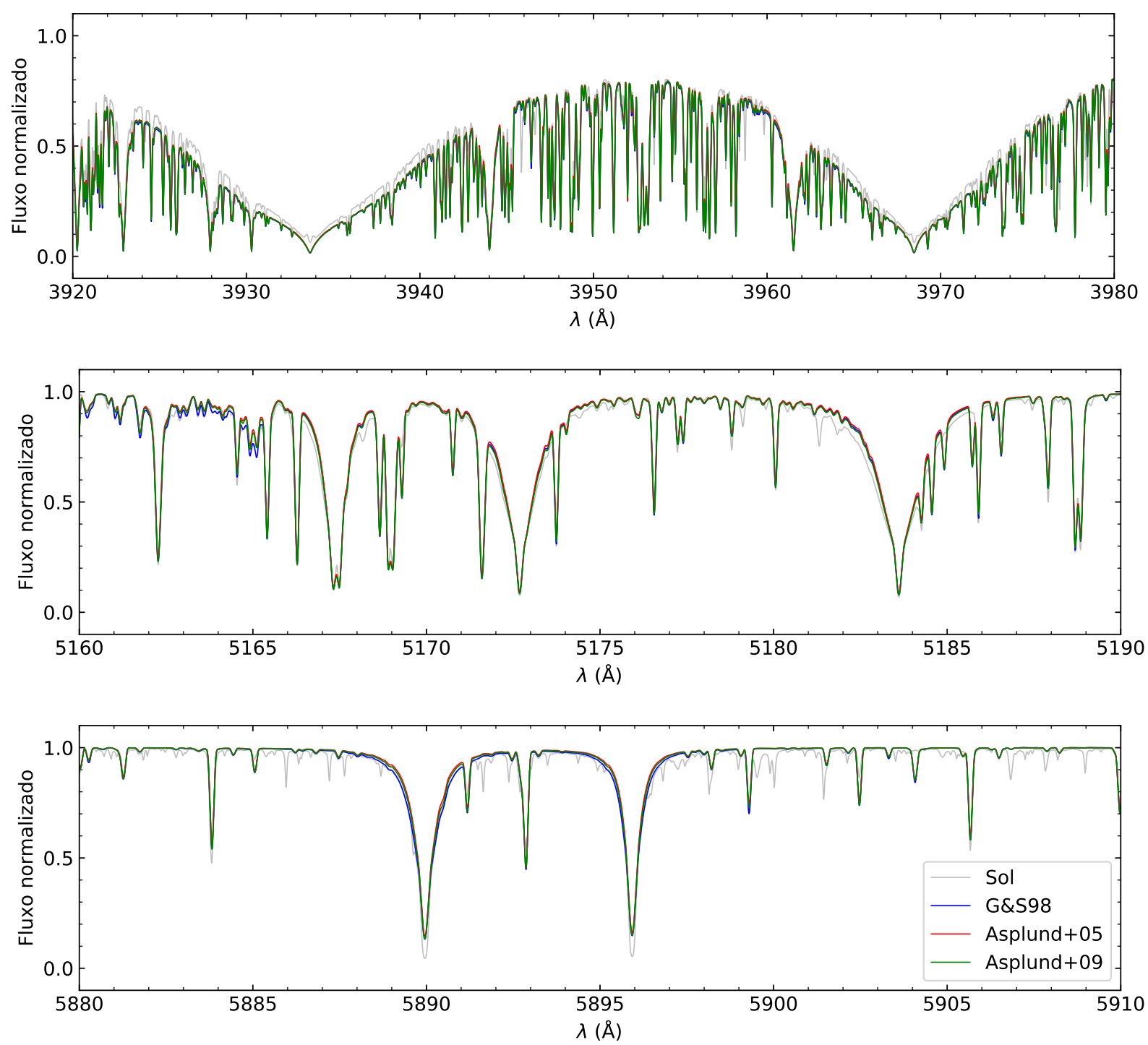

Figura 3.3: Espectros sintéticos solares calculados com diferentes abundâncias: Grevesse e Sauval (1998) em azul, Asplund et al. (2005) em vermelho e Asplund et al. (2009) em verde, em comparação ao espectro observado do Sol (Kurucz (2005b) em preto), nas regiões características de Ca II [H-K] (cima), Mg T (meio) e $\mathrm{Na} D$ (baixo). 


\subsubsection{Opacidades moleculares adotadas}

As opacidades moleculares usadas neste projeto foram obtidas do website de R. Ku-

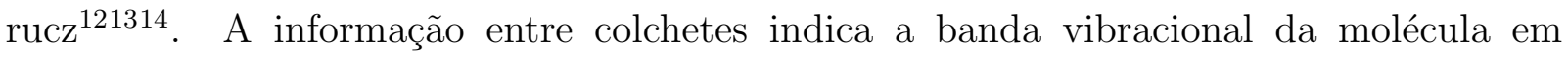
questão, quando explícita pelo autor. À época da busca, encontramos mais de uma lista de opacidades para algumas das espécies moleculares disponíveis nos diretórios do website. Na Tabela 3.2 listamos esses casos, identificando os diferentes arquivos pela coluna "ID" e indicando em "Referência" o endereço eletrônico de cada um.

Tabela 3.2 - Lista de moléculas com mais de um arquivo disponível.

\begin{tabular}{|c|c|c|}
\hline Molécula & ID & Arquivo \\
\hline \multirow{2}{*}{$\mathrm{C}_{2}[\mathrm{D}-\mathrm{A}]$} & Kurucz & http://kurucz.harvard.edu/linelists/linesmol/c2da.asc \\
\hline & Brooke & http://kurucz.harvard.edu/molecules/c2/c2dabrookek.asc \\
\hline \multirow{2}{*}{$\mathrm{SiH}$} & Antigo & http://kurucz.harvard.edu/linelists/linesmol/sih.asc \\
\hline & Novo & http://kurucz.harvard.edu/linelists/linesmol/sihnew.asc \\
\hline \multirow{3}{*}{$\mathrm{OH}$} & Antigo & http://kurucz.harvard.edu/linelists/linesmol/oh.asc \\
\hline & Novo & http://kurucz.harvard.edu/linelists/linesmol/ohnew.asc \\
\hline & Atualizado & http://kurucz.harvard.edu/molecules/oh/ohupdate.asc \\
\hline \multirow{2}{*}{$\mathrm{CN}[\mathrm{A}-\mathrm{X}]$} & Kurucz & http://kurucz.harvard.edu/linelists/linesmol/chax.asc \\
\hline & Brooke & http://kurucz.harvard.edu/molecules/cn/cnaxbrookek. asc \\
\hline \multirow{2}{*}{$\mathrm{CN}[\mathrm{B}-\mathrm{X}]$} & Kurucz & http://kurucz.harvard.edu/linelists/linesmol/chbx.asc \\
\hline & Brooke & http://kurucz.harvard.edu/molecules/cn/cnbxbrookek.asc \\
\hline \multirow{3}{*}{$\mathrm{CH}$} & Kurucz & http://kurucz.harvard.edu/linelists/linesmol/ch.asc \\
\hline & Jorgensen & http://kurucz.harvard.edu/molecules/ch/chjorg.asc \\
\hline & Masseron & http://kurucz.harvard.edu/molecules/ch/chmasseron.asc \\
\hline
\end{tabular}

\footnotetext{
12 http://kurucz.harvard.edu/linelists/linesmol/ $\mathrm{C}_{2}([\mathrm{~A}-\mathrm{X}],[\mathrm{D}-\mathrm{A}],[\mathrm{E}-\mathrm{A}]), \mathrm{CH}([\mathrm{A}-\mathrm{X}],[\mathrm{B}-\mathrm{X}],[\mathrm{C}-\mathrm{X}]), \mathrm{CN}([\mathrm{A}-\mathrm{X}],[\mathrm{B}-$ $\mathrm{X}]), \mathrm{CO}([\mathrm{A}-\mathrm{X}],[\mathrm{X}-\mathrm{X}]), \mathrm{H}_{2}([\mathrm{~B}-\mathrm{X}],[\mathrm{C}-\mathrm{X}],[\mathrm{X}-\mathrm{X}]), \mathrm{Hd}[\mathrm{X}-\mathrm{X}], \mathrm{MgH}([\mathrm{A}-\mathrm{X}],[\mathrm{B}-\mathrm{X}]), \mathrm{NH}, \mathrm{OH}, \mathrm{SiH}, \mathrm{SiO}([\mathrm{A}-\mathrm{X}],[\mathrm{E}-\mathrm{X}],[\mathrm{X}-\mathrm{X}])$.

13 http://kurucz.harvard.edu/molecules/ $\mathrm{AlH}([\mathrm{A}-\mathrm{X}],[\mathrm{X}-\mathrm{X}]), \mathrm{AlO}, \mathrm{C}_{2}[\mathrm{D}-\mathrm{A}], \mathrm{CaH}([\mathrm{A}-\mathrm{X}],[\mathrm{E}-\mathrm{X}],[\mathrm{X}-\mathrm{X}]), \mathrm{CaO}, \mathrm{CH}$, CN[A-X], CN[B-X], CO[A-X], CrH[A-X], FeH[F-X], H2[X-X], H2O, MgH([A-X], [B-X], [X-X]), MgO, NaH, OH, SiH([A$\mathrm{X}],[\mathrm{X}-\mathrm{X}]), \operatorname{TiH}([\mathrm{A}-\mathrm{X}],[\mathrm{B}-\mathrm{X}]), \mathrm{TiO}, \mathrm{VO}([\mathrm{A}-\mathrm{X}],[\mathrm{B}-\mathrm{X}],[\mathrm{C}-\mathrm{X}])$.

14 Último acesso em: 28-02-2020.
} 
A Figura 3.4 ilustra o espectro sintético calculado único e exclusivamente com cada arquivo das espécies moleculares da Tabela 3.2 (i.e., uma de cada vez, sem incluir outras listas de opacidades moleculares e atômicas). Para visualização, todos os espectros sintéticos em 3.4 foram calculados com resolução amostra $15 R_{\lambda}=600000$, degradados para resolução espectral $R_{\mathrm{LSF}}=300000$ de Kurucz $(2005 \mathrm{~b})$, com abundâncias químicas de Asplund et al. (2005), e degradados arbitrariamente por um filtro gaussiand ${ }^{16} \operatorname{com} \sigma=30$ para facilitar a visualização. Dos paineis na Fig. 3.4 notamos a influência própria de cada espécie molecular no espectro sintético.
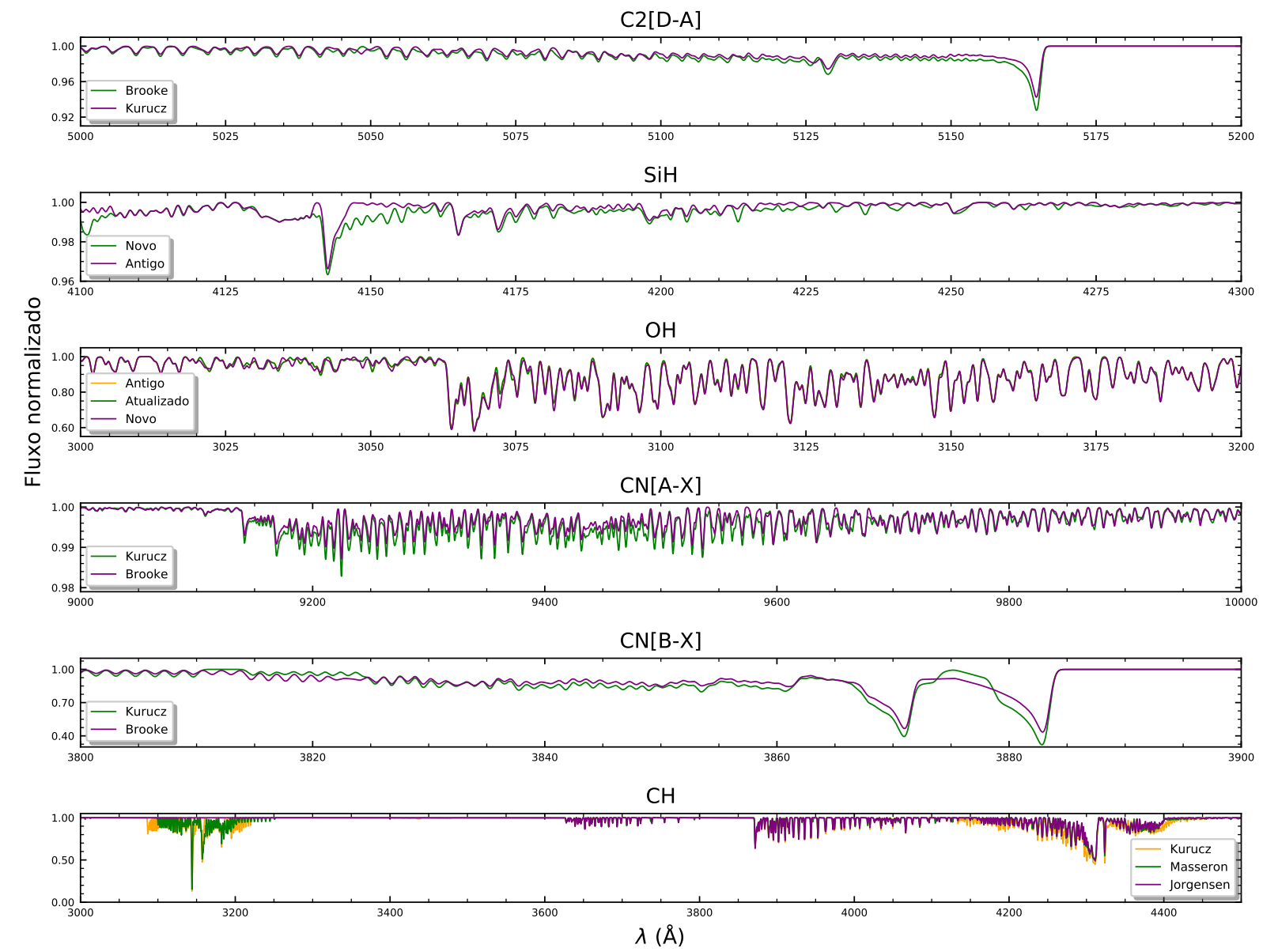

Figura 3.4: Comparação entre as listas de opacidades moleculares relacionadas na Tabela 3.2 , De cima para baixo: $\mathrm{C}_{2}[\mathrm{D}-\mathrm{A}], \mathrm{SiH}, \mathrm{OH}, \mathrm{CN}[\mathrm{A}-\mathrm{X}], \mathrm{CN}[\mathrm{B}-\mathrm{X}]$ e $\mathrm{CH}$.

\footnotetext{
${ }^{15}$ I.e., calculado com passo em comprimento de onda $\Delta \lambda$ tal que $R_{\lambda}=\lambda / \Delta \lambda=600000$.

16 Método gaussian_filter, do pacote SciPy (Virtanen et al. 2020) no PYTHON.
} 
A fim de escolher dentre as opções da Tabela 3.2 com as estrelas de referência, calculamos espectros adotando abundância solar por Asplund et al. (2005) e listas de opacidades moleculares $\}^{17}$ e atômicas por R. Kurucz $2^{18}$ (Kurucz, 2018) e comparamos com espectro observado do Sol por Kurucz (2005b). O Atlas do Fluxo Solar (tradução livre) de Wallace et al. (2011), que possui resolução espectral de 348000 a 676000 , a depender do intervalo de comprimento de onda, de 2958,6 a 9259,3 A, identifica muitas espécies moleculares e atômicas ao longo do espectro. Utilizamos este Atlas para nos auxiliar na seleção entre as listas disponíveis, comparando os diferentes espectros em regiões cuja influência do fluxo de cada molécula é importante.

\subsubsection{1 $C_{2}$}

Do website de R. Kurucz encontramos listas de linhas moleculares para $\mathrm{C}_{2}$ separadas por transições moleculares e bandas vibracionais, mas somente $\mathrm{C}_{2}[\mathrm{D}-\mathrm{A}]$ possui dois arquivos, "c2da.asc" e "c2dabrookek.asc", que chamamos de "Kurucz" e "Brooke", respectivamente, conforme a Tabela 3.2. De acordo com o documento "c2da.readme"19, a lista molecular "Kurucz" foi reformatada com base em Brooke et al. (2013), substituindo as linhas de opacidade da banda vibracional $\mathrm{C}_{2}[\mathrm{D}-\mathrm{A}](1-2)$. O espectro referente a cada arquivo é ilustrado na Figura 3.5, calculados somente com a abundância química solar de Asplund et al. (2005).

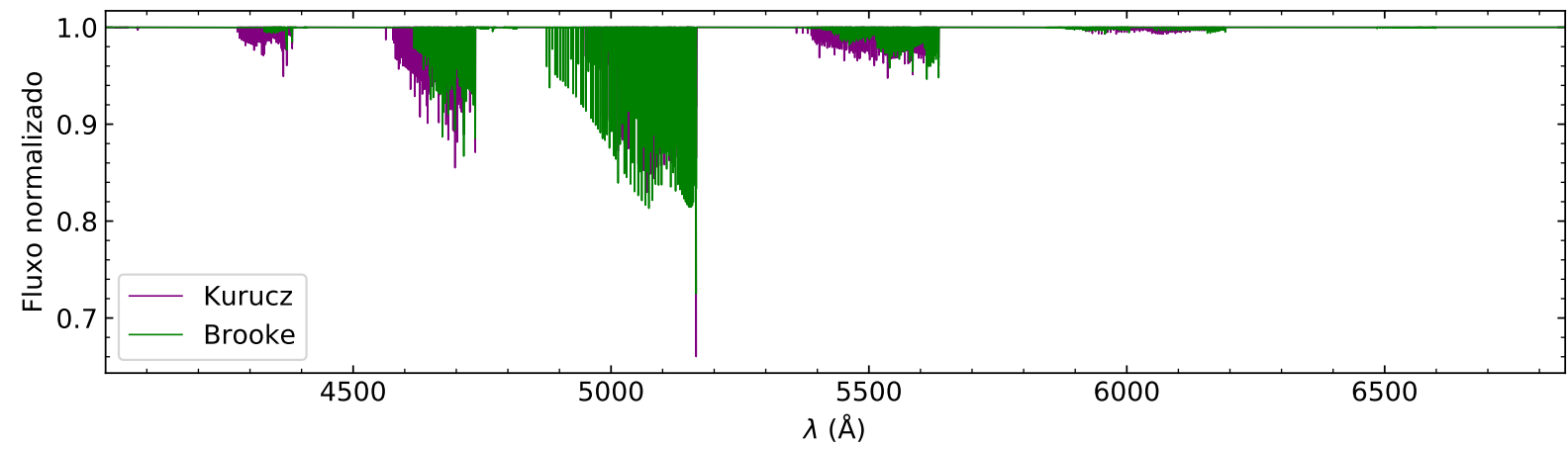

Figura 3.5: Espectros sintéticos das listas de opacidade da molécula $\mathrm{C}_{2}[\mathrm{D}-\mathrm{A}]$ "Kurucz" (roxo) e "Brooke" (verde), conforme Tabela 3.2, de 4020 a $6850 \AA$.

\footnotetext{
17 indicadas na nota de rodapé 11, desta subseção.

18 http://kurucz.harvard.edu/linelists/gfnew/gfall08oct17.dat acessado em: 08-08-2018.

19 http://kurucz.harvard.edu/molecules/c2/c2da.readme
} 
Os paineis da Figura 3.6 ilustram o efeito de cada lista de opacidade de $\mathrm{C}_{2}[\mathrm{D}-\mathrm{A}]$ no espectro sintético em comparação ao espectro observado do Sol. Os espectros sintéticos e observado foram convoluídos para uma menor resolução para conseguirmos visualizar alguma diferença entre as listas (Figura 3.6, painel superior). Mesmo aproximando na região em que é possível fazer distinção (Figura 3.6, painel inferior), não é fácil afirmar visualmente qual dos dois espectros sintéticos melhor representa o espectro observado do Sol. Por este motivo, optamos por adotar o espectro sintético cuja lista de opacidade molecular de $\mathrm{C}_{2}[\mathrm{D}-\mathrm{A}]$ é a mais atual, ou seja, a que contém as modificações de Brooke et al. (2013) ("Brooke").
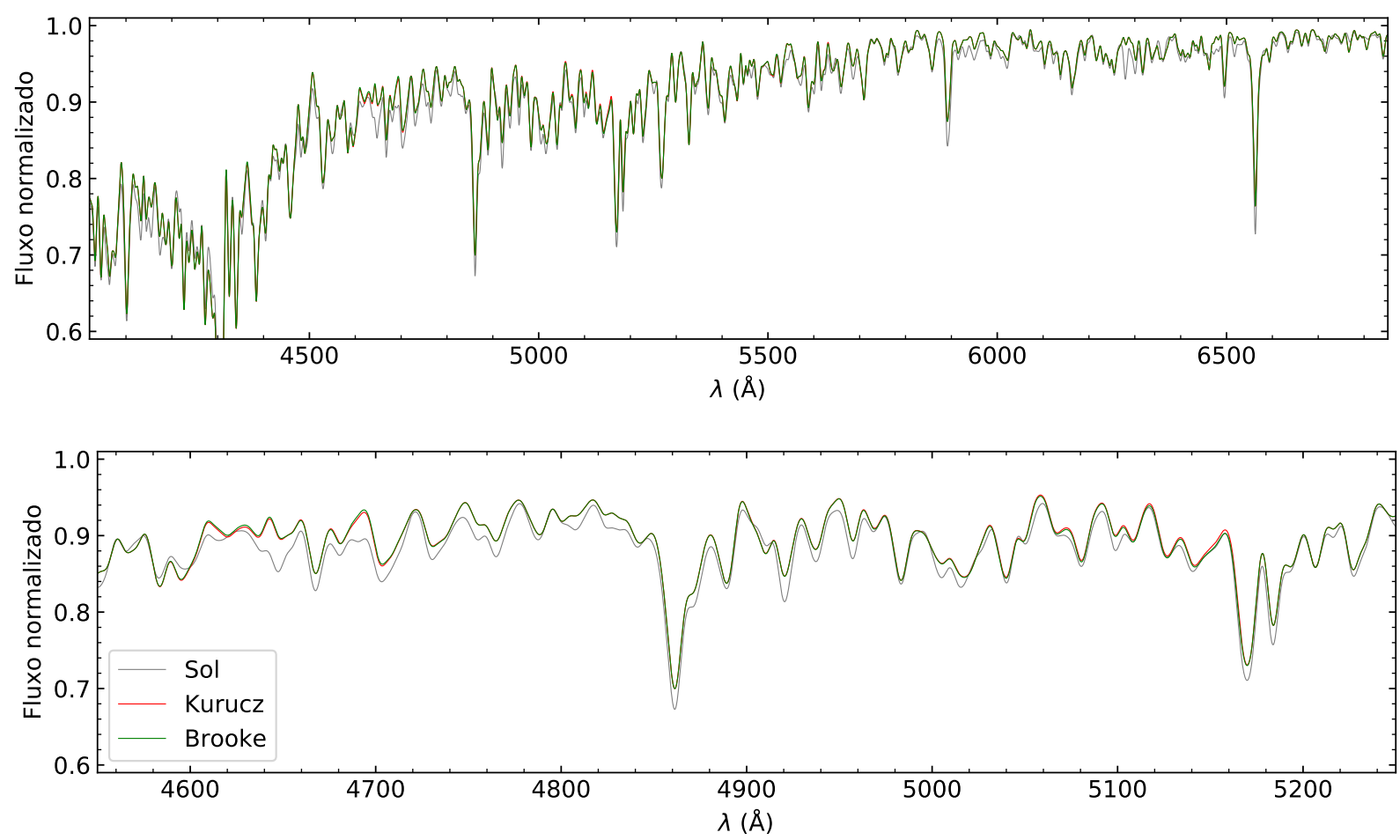

Figura 3.6: Espectros sintéticos das listas de opacidade da molécula $\mathrm{C}_{2}[\mathrm{D}-\mathrm{A}]$ "Kurucz" (vermelho) e "Brooke" (verde), conforme Tabela 3.2. comparados com o espectro observado do Sol por Kurucz (2005b) (cinza), de 4020 a $6850 \AA$ (painel superior) e de 4550 a $5250 \AA$ (painel inferior). 


\subsubsection{2 $\mathrm{SiH}$}

Para SiH encontramos duas versões de lista de linhas moleculares, "sih.asc" e "sihnew.asc", identificados neste trabalho por "Antigo" e "Novo", respectivamente (vide Tabela 3.2. . Conforme o documento "sihax.readme" 20 na versão "sihnew.asc" foram atualizados os níveis de energia da transição molecular $\mathrm{SiH}[\mathrm{A}-\mathrm{X}]$ (Ram et al., 1998). O espectro referente a cada arquivo é ilustrado na Figura 3.7, calculados somente com a abundância química solar de Asplund et al. (2005).

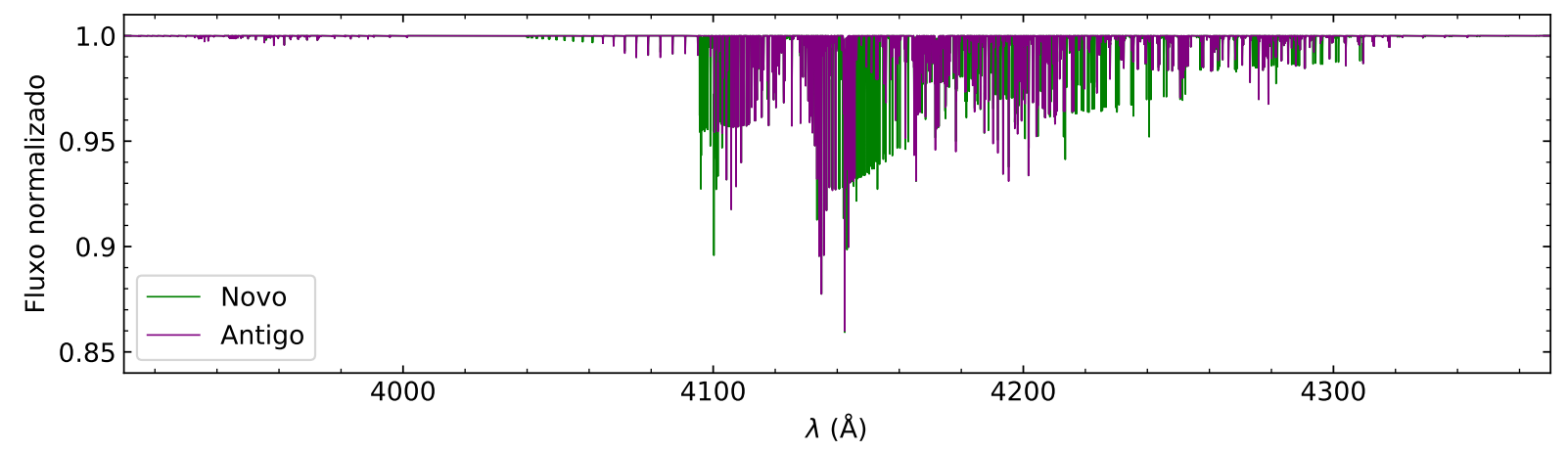

Figura 3.7: Espectros sintéticos das listas de opacidade da molécula SiH "Antigo" (roxo) e "Novo" (verde), conforme Tabela 3.2 de 3910 a $4370 \AA$.

Os paineis da Figura 3.8 ilustram o efeito de cada lista de opacidade de $\mathrm{SiH}$ no espectro sintético em comparação ao espectro observado do Sol. Os espectros sintéticos e observado foram convoluídos para uma menor resolução para conseguirmos visualizar alguma diferença entre as listas (Figura 3.8, painel superior). Apesar de ser difícil a distinção, pelo painel inferior da Figura 3.8, é possível afirmar visualmente que espectro sintético "Novo", em verde, está mais próximo do espectro observado do Sol do que o espectro sintético "Antigo". Por este motivo, optamos por adotar o espectro sintético cuja lista de opacidade molecular de SiH é a mais atual, ou seja, a que contém as atualizações dos níveis de energia [A-X] ("Novo").

20 http://kurucz.harvard.edu/molecules/sih/sihax.readme 

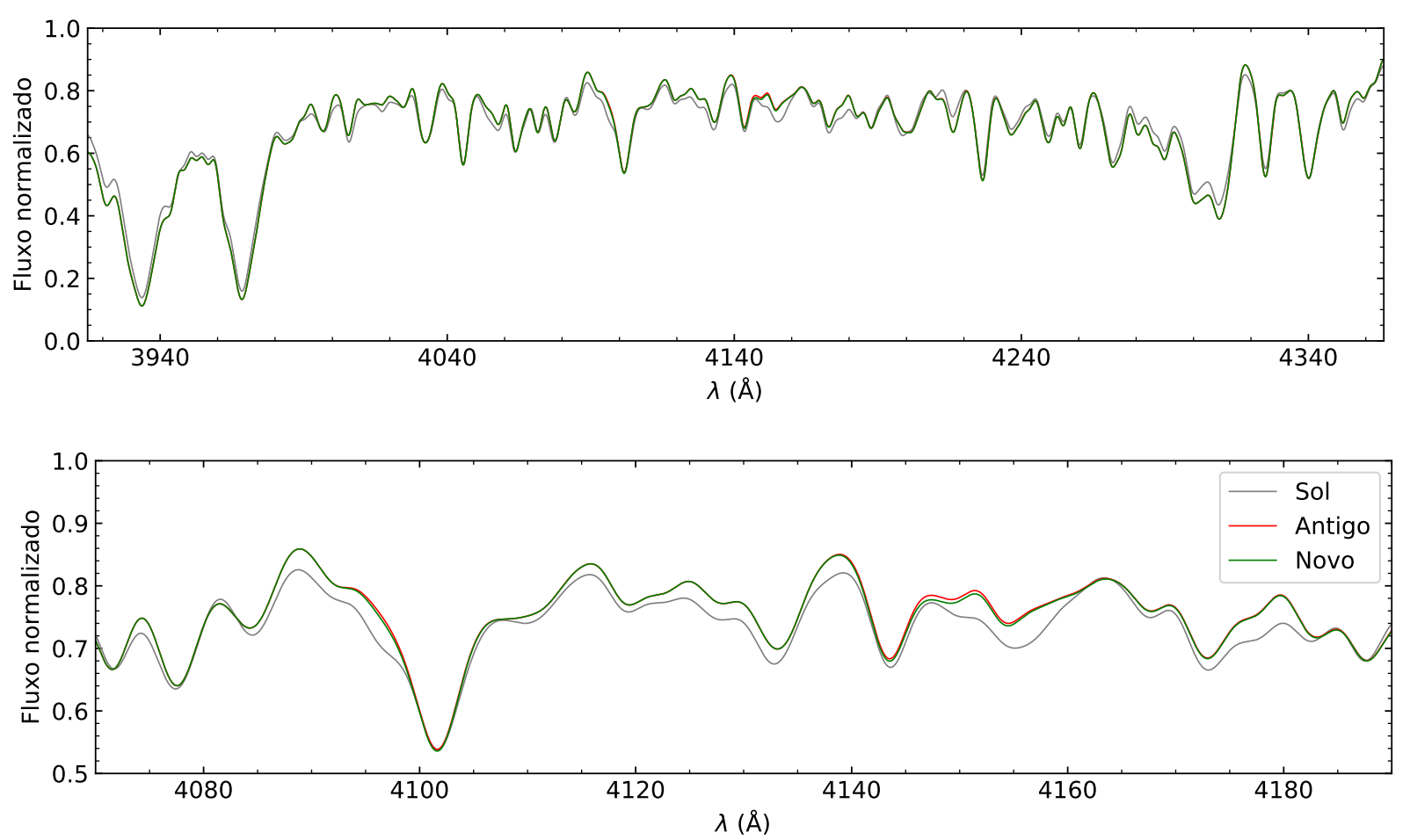

Figura 3.8: Espectros sintéticos das listas de opacidade da molécula SiH "Antigo" (roxo) e "Novo" (verde), conforme Tabela 3.2, comparados com o espectro observado do Sol por Kurucz (2005b) (cinza), de 3910 a $4370 \AA$ (painel superior) e de 4080 a $4180 \AA$ (painel inferior).

\subsubsection{3 $\mathrm{OH}$}

Encontramos três versões de lista de linhas moleculares para $\mathrm{OH}$, isto é, os documentos disponíveis são diferenciados pelos nomes "oh.asc", "ohnew.asc" e "ohupdate.asc", e identificados neste trabalho como "Antigo", "Novo" e "Atualizado", respectivamente, conforme Tabela 3.2. De acordo com o documento "ohax.readme" 21] é mencionada a atualização dos níveis de energia para a transição molecular OH [A-X] (Bernath e Colin, 2009; Yousefi et al., 2018). O espectro referente a cada arquivo é ilustrado na Figura 3.9, calculados somente com a abundância química solar de Asplund et al. (2005).

Os paineis da Figura 3.10 ilustram o efeito de cada lista de opacidade de $\mathrm{OH}$ no espectro sintético em comparação ao espectro observado do Sol. Pelo painel inferior de 3.10 , não é possível distinguir o fluxo do espectro sintético referente ao arquivo $\mathrm{OH}$ "Antigo" do fluxo referente ao arquivo "Novo", sendo considerados espectros idênticos. É possível afirmar

21 http://kurucz.harvard.edu/molecules/oh/ohax.readme 
visualmente que o espectro sintético "Novo" está mais próximo do espectro observado do Sol do que o "Atualizado", mas a diferença entre os dois espectros é muito pequena e, também, vemos mais ou menos diferenças a depender da convolução aplicada nos espectros. Dada a degenerecência entre as opacidades moleculares e atômicas, optamos por adotar o espectro sintético cuja lista de opacidade molecular de $\mathrm{OH}$ é a mais atual, ou seja, a que contém as atualizações dos níveis de energia [A-X] (i.e., "Atualizado").

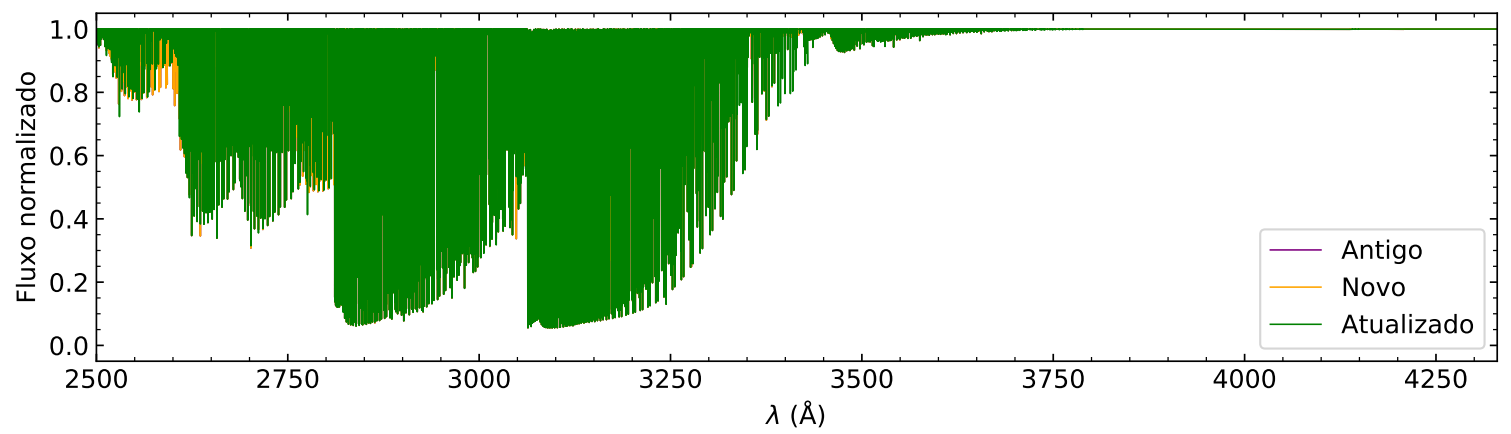

Figura 3.9: Espectros sintéticos das listas de opacidade da molécula OH "Antigo" (roxo), "Novo" (laranja) e "Atualizado" (verde), conforme Tabela 3.2 de 2500 a $4330 \AA$.
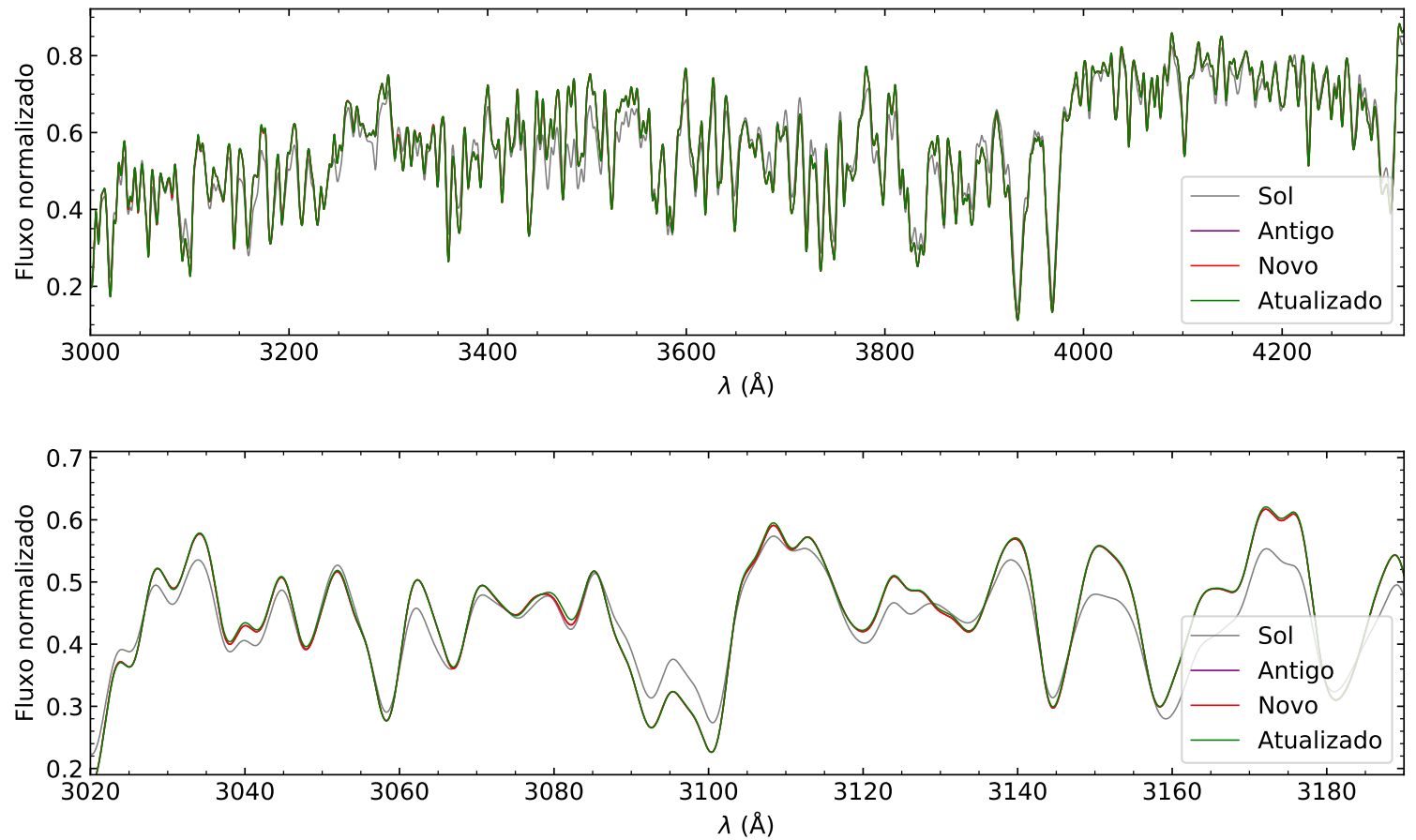

Figura 3.10: Espectros sintéticos das listas de opacidade da molécula OH "Antigo" (roxo), "Novo" (vermelho) e "Atualizado" (verde), conforme Tabela 3.2, comparados com o espectro observado do Sol por Kurucz 2005b) (cinza), de 3000 a $4330 \AA$ (painel superior) e de 3020 a $3190 \AA$ (painel inferior). 


\subsubsection{CN}

A molécula de CN é separada pelas transições moleculares $[\mathrm{A}-\mathrm{X}]$ e $[\mathrm{B}-\mathrm{X}]$, cada uma com duas versões de listas de opacidades disponíveis. Para a transição [A-X], as listas são diferenciadas pelos nomes "cnax.asc" e "cnaxbrookek.asc", identificadas neste trabalho como "Kurucz" e "Brooke", respectivamente, conforme a Tabela 3.2. Para a transição [B-X], elas são diferenciadas pelos nomes "cnbx.asc' e "cnbxbrookek.asc" e, da mesma Tabela 3.2 , são identificadas também como "Kurucz" e "Brooke".

Segundo o documento "cnbrooke.readme" 22, as listas "Kurucz" foram atualizadas com base em Brooke et al. (2014). As transições moleculares [A-X] e [B-X] atuam em diferentes regiões do espectro eletromagnético, como mostram os paineis superior e inferior, respectivamente, da Figura 3.11. Em ambos os paineis, o espectro referente a cada arquivo foi calculado somente com a abundância química solar de Asplund et al. (2005) e, como vemos na Figura 2.3, as linhas telúricas são visualmente fortes a partir de $\sim 5500 \AA$. Por isso, começamos nossa análise com CN $[\mathrm{A}-\mathrm{X}]$.
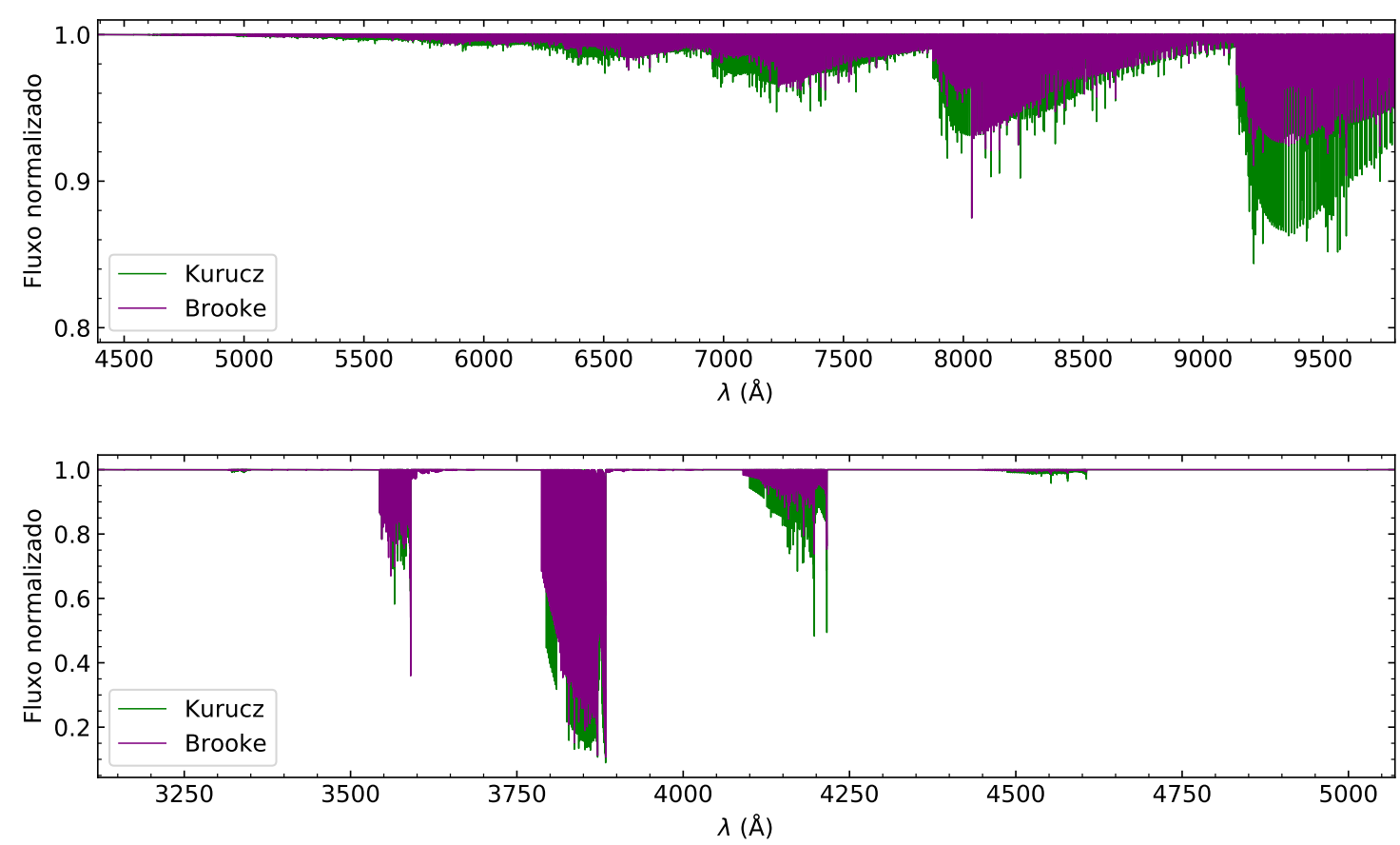

Figura 3.11: Espectros sintéticos das listas de opacidade "Brooke" (roxo) e "Kurucz" (verde), conforme Tabela 3.2 da molécula CN [A-X] de 4390 a $10000 \AA$ (painel superior) e CN [B-X] de 3120 a $5070 \AA$ (painel inferior).

22 http://kurucz.harvard.edu/molecules/cn/cnbrooke.readme 
Os paineis na Figura 3.12 ilustram os espectros sintéticos de cada lista de opacidade de $\mathrm{CN}[\mathrm{A}-\mathrm{X}]$, em comparação ao espectro observado do Sol. A análise de CN $[\mathrm{A}-\mathrm{X}]$ é bastante afetada pelas linhas telúricas, o que pode ser visto no painel superior de 3.12 . Da Tabela 2 em Wallace et al. (2011), a banda vibracional de CN [A-X] com maior impacto no espectro do fluxo solar é a (1-0), correspondendo ao intervalo 9140 a $9255 \AA$. Assim, no painel inferior de 3.12 , ainda que visualmente os espectros sintéticos não representem bem o espectro observado, é possível afimar que o espectro sintético calculado com "Kurucz" está mais próximo do observado do que o calculado com "Brooke".
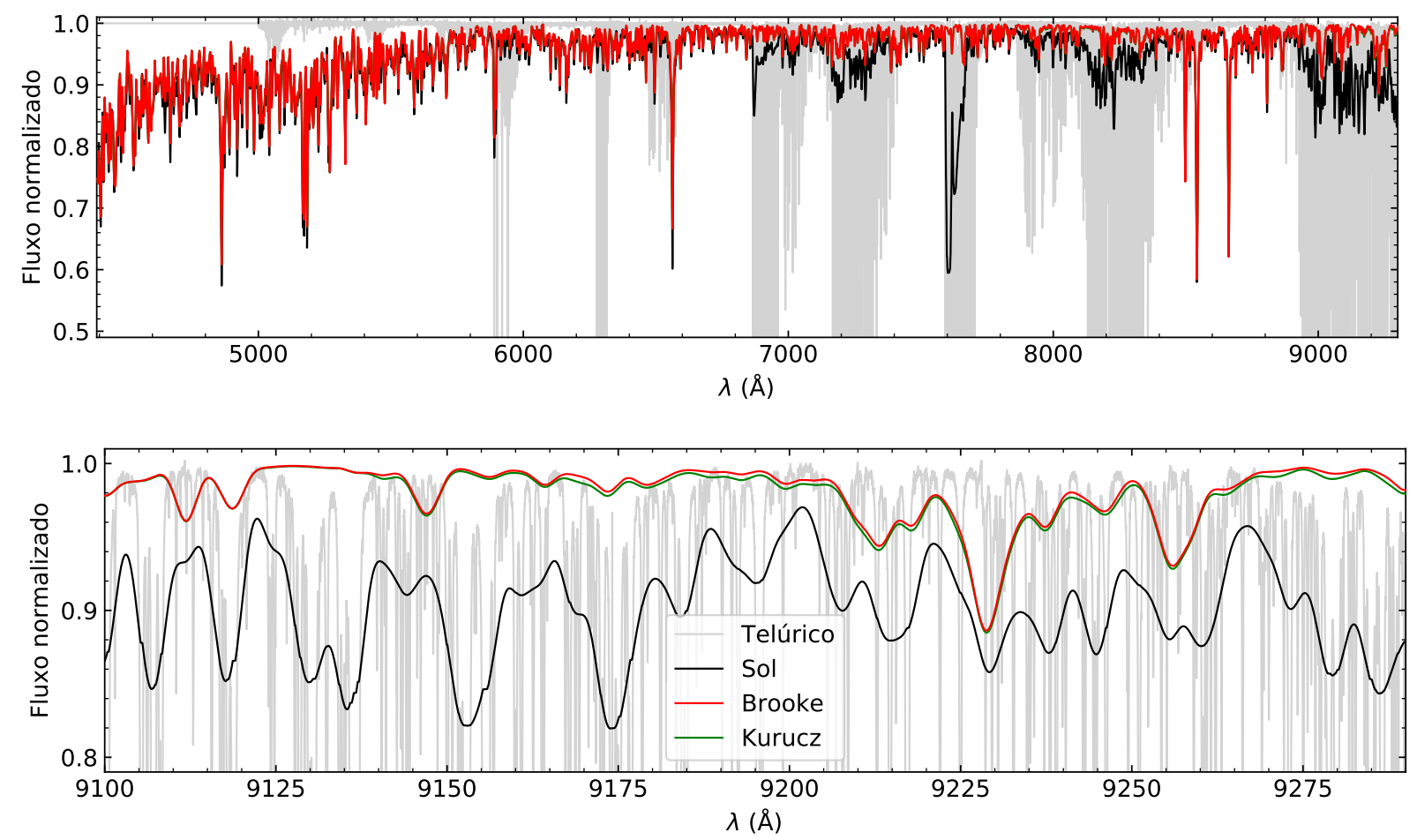

Figura 3.12: Espectros sintéticos das listas de opacidade da molécula CN [A-X] "Kurucz" (verde) e "Brooke" (vermelho), conforme Tabela 3.2. comparados com o espectro telúrico por Hinkle et al. (2000) (cinza claro) e o espectro observado do Sol por Kurucz (2005b) (preto), de 4390 a $9300 \AA$ (painel superior) e de 9100 a $9300 \AA$ (painel inferior). Em ambos os gráficos, para melhor visualização, os espectros sintéticos e o observado do Sol foram degradados e foram desconsiderados os elementos em comprimento de onda do espectro do Sol cujo fluxo telúrico normalizado fosse menor do que 0,5 .

Para $\mathrm{CN}[\mathrm{B}-\mathrm{X}]$ o método de escolha seguiu praticamente o mesmo procedimento adotado com as outras moléculas até aqui, exceto CN $[\mathrm{A}-\mathrm{X}]$. Da Tabela 2 de Wallace et al. (2011), a região destacada para o CN[B-X] compreende o intervalo de 3120 a $5070 \AA$. Neste intervalo, o impacto da contaminação telúrica não é observado tal como foi com CN [A- 
$\mathrm{X}]$ e, por este motivo, somente degradamos os espectros para ilustrar as diferenças. Na Figura 3.13 , os paineis ilustram o espectro sintético das duas listas de $\mathrm{CN}[\mathrm{B}-\mathrm{X}]$. Com a aproximação dada na região de destaque do $\mathrm{CN}[\mathrm{B}-\mathrm{X}]$ (painel inferior), percebemos que ao longo do comprimento de onda varia o espectro sintético que melhor representa o espectro observado, impedindo que um deles fosse escolhido visualmente.
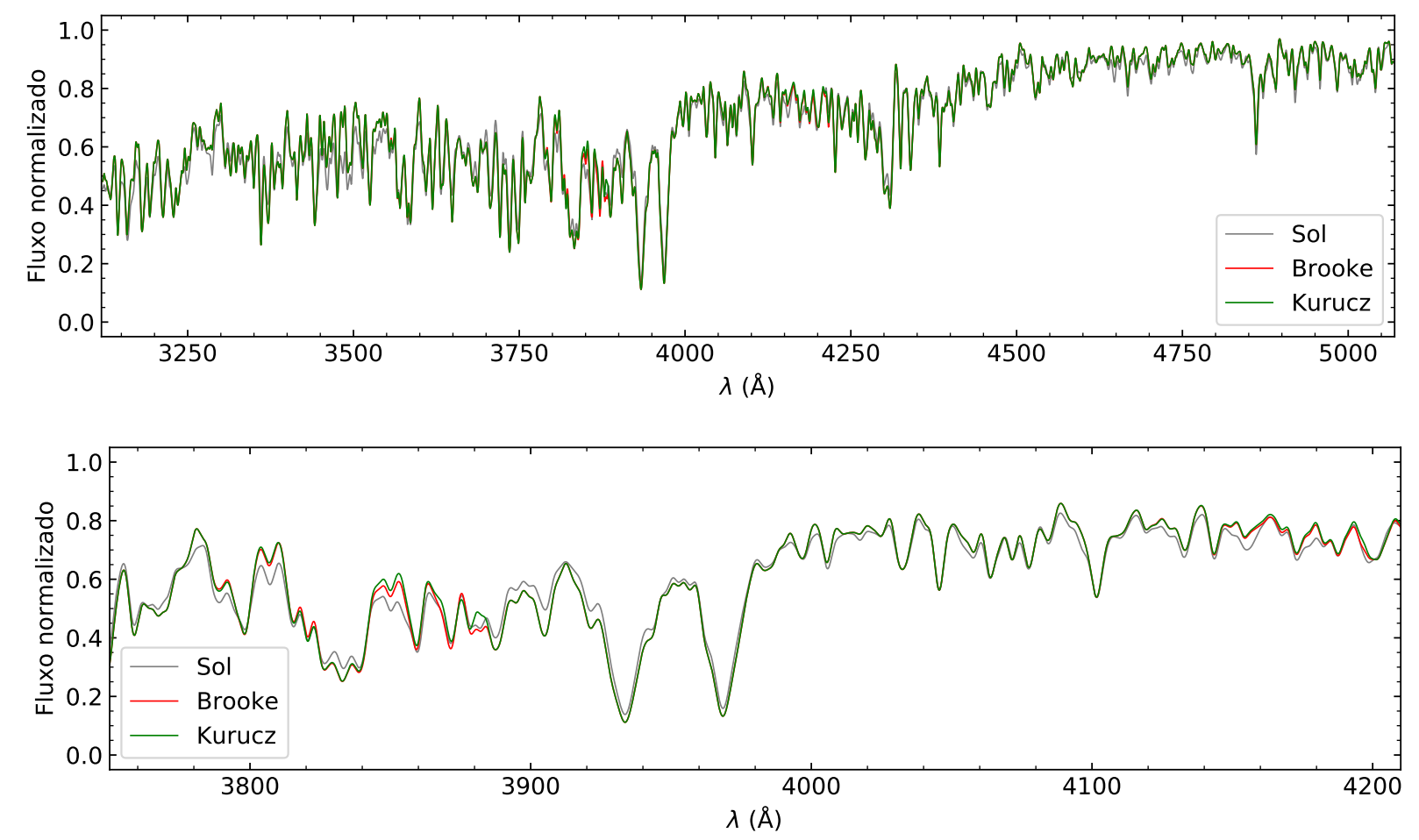

Figura 3.13: Espectros sintéticos das listas de opacidade da molécula CN [B-X] "Kurucz" (verde) e "Brooke" (vermelho), conforme Tabela 3.2, comparados com o espectro observado do Sol por Kurucz (2005b) (cinza), de 3120 a $5070 \AA$ (cima) e de 3770 a $4220 \AA$ (baixo).

De acordo com a Tabela 2 em Wallace et al. (2011), a banda vibracional de CN [B$\mathrm{X}$ ] destacada com maior impacto no espectro do fluxo solar é a (0-0), correspondendo ao intervalo de 3742 a $3883 \AA$ A. Desta forma, nos paineis da Figura 3.14 mostramos o espectro sintético de cada lista em um intervalo arbitrário de $30 \AA$ na região de $\mathrm{CN}[\mathrm{B}-\mathrm{X}](0-0)$. Notamos que mesmo em um intervalo muito pequeno de comprimento de onda, ora o espectro sintético de uma lista representa melhor o espectro observado, ora o outro. Dada a escolha feita para CN [A-X] (i.e., "Kurucz"), por consistência de autoria, escolhemos a lista de opacidades "Kurucz" para CN [B-X], também. 

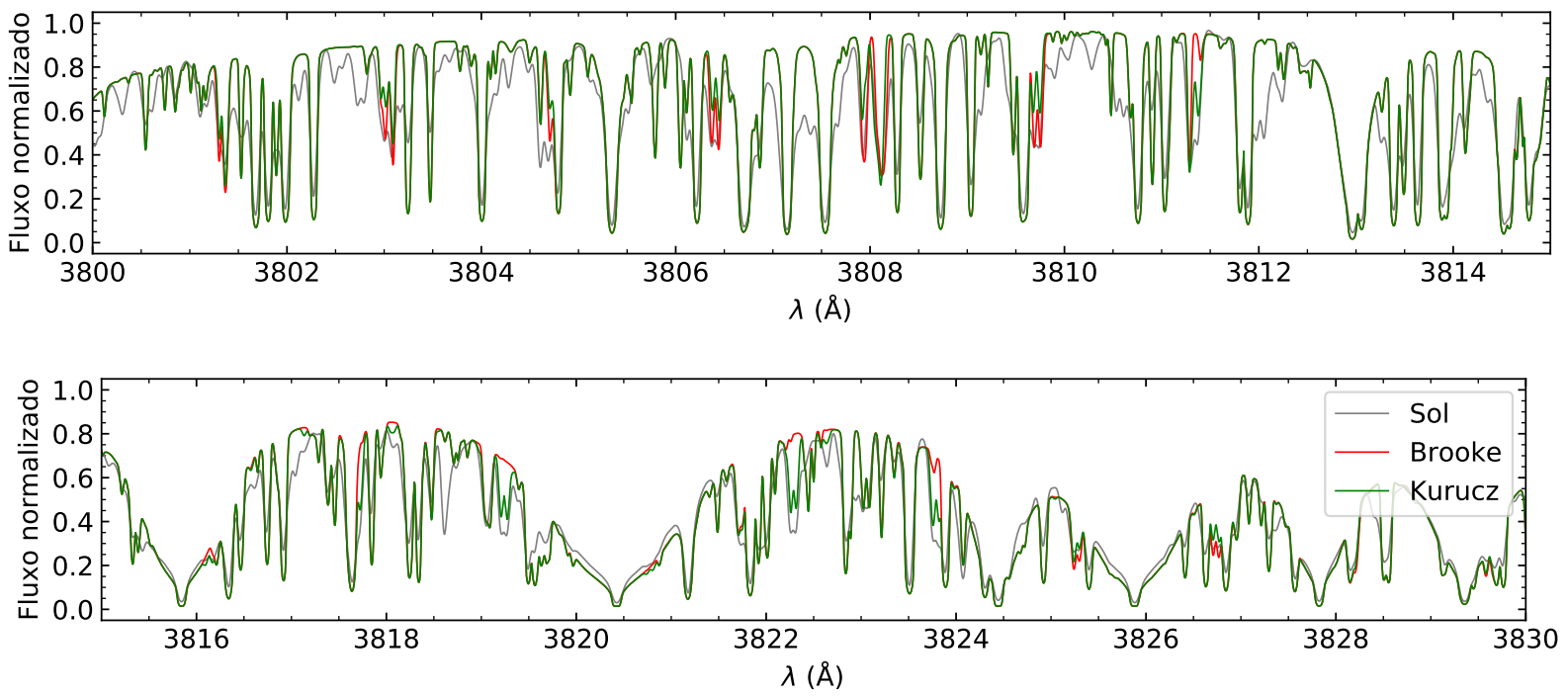

Figura 3.14: Painel de espectros sintéticos não-convoluídos das listas de opacidade da molécula CN[B-X] "Kurucz" (verde) e "Brooke" (vermelho), conforme Tabela 3.2 comparados com o espectro observado do Sol por Kurucz (2005b) (cinza), de 3800 a $3830 \AA$.

\subsubsection{CH}

Do website de R. Kurucz encontramos três versões de lista de opacidade molecular para o CH: "ch.asc", "chjorg.asc" e "chmasseron.asc", os quais identificamos neste trabalho como "Kurucz", "Jorgensen" e "Masseron", respectivamente, conforme Tabela 3.2. Nos arquivos "chjorgensen.readme" "23 e "chmasseron.readme" 4 são mencionadas alterações na lista "Kurucz", uma com base em Jorgensen et al. (1996) ("Jorgensen") e outra em Masseron et al. (2014) ("Masseron"). O espectro referente a cada arquivo é ilustrado na Figura 3.15. calculados somente com a abundância química solar de Asplund et al. (2005).

Para tentar verificar as diferenças entre as listas, os paineis da Figura 3.16 ilustram o efeito de cada lista de opacidade de $\mathrm{CH}$ no espectro sintético em comparação ao espectro observado do Sol. Em ambos os gráficos, os espectros foram convoluídos para facilitar ilustração. Da Tabela 2 de Wallace et al. (2011), no painel inferior de 3.16 mostramos os espectros no intervalo em comprimento de onda em que as transições moleculares $[\mathrm{A}-\mathrm{X}]$, [B-X] e [C-X] de CH são destacadas. Neste intervalo verificamos uma maior discrepância do impacto que cada lista de opacidade molecular tem no fluxo do espectro sintético.

\footnotetext{
23 http://kurucz.harvard.edu/molecules/ch/chjorgensen.readme

24 http://kurucz.harvard.edu/molecules/ch/chmasseron.readme
} 


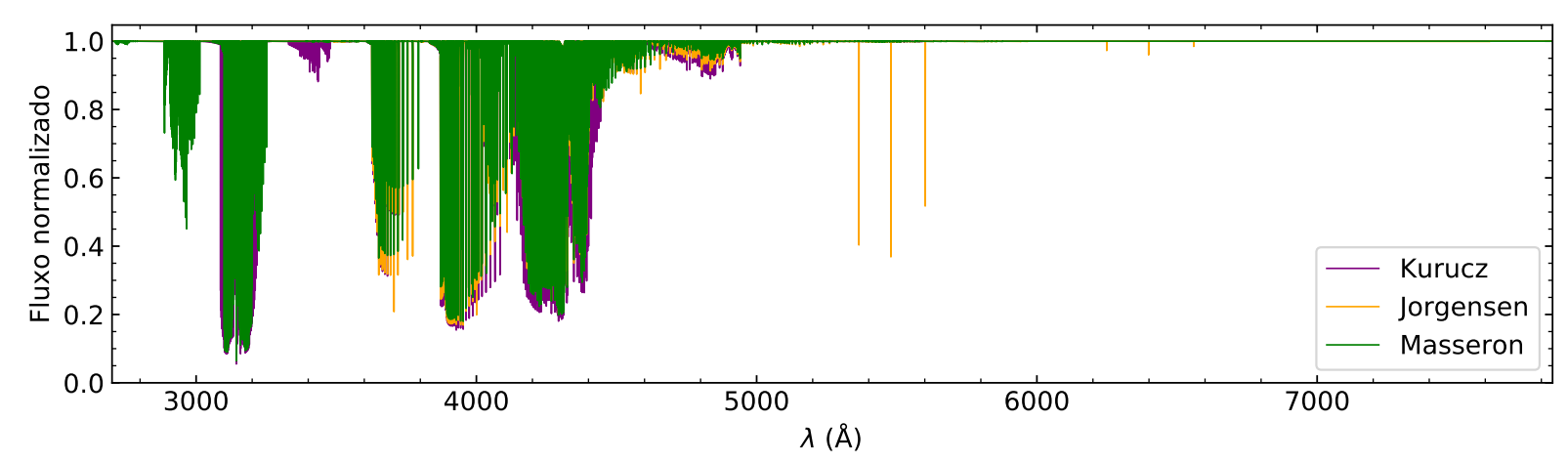

Figura 3.15: Espectros sintéticos das listas de opacidade da molécula CH "Kurucz" (roxo), "Jorgensen"(vermelho) e "Masseron" (verde), conforme Tabela 3.2, de 2700 a $7840 \AA$.
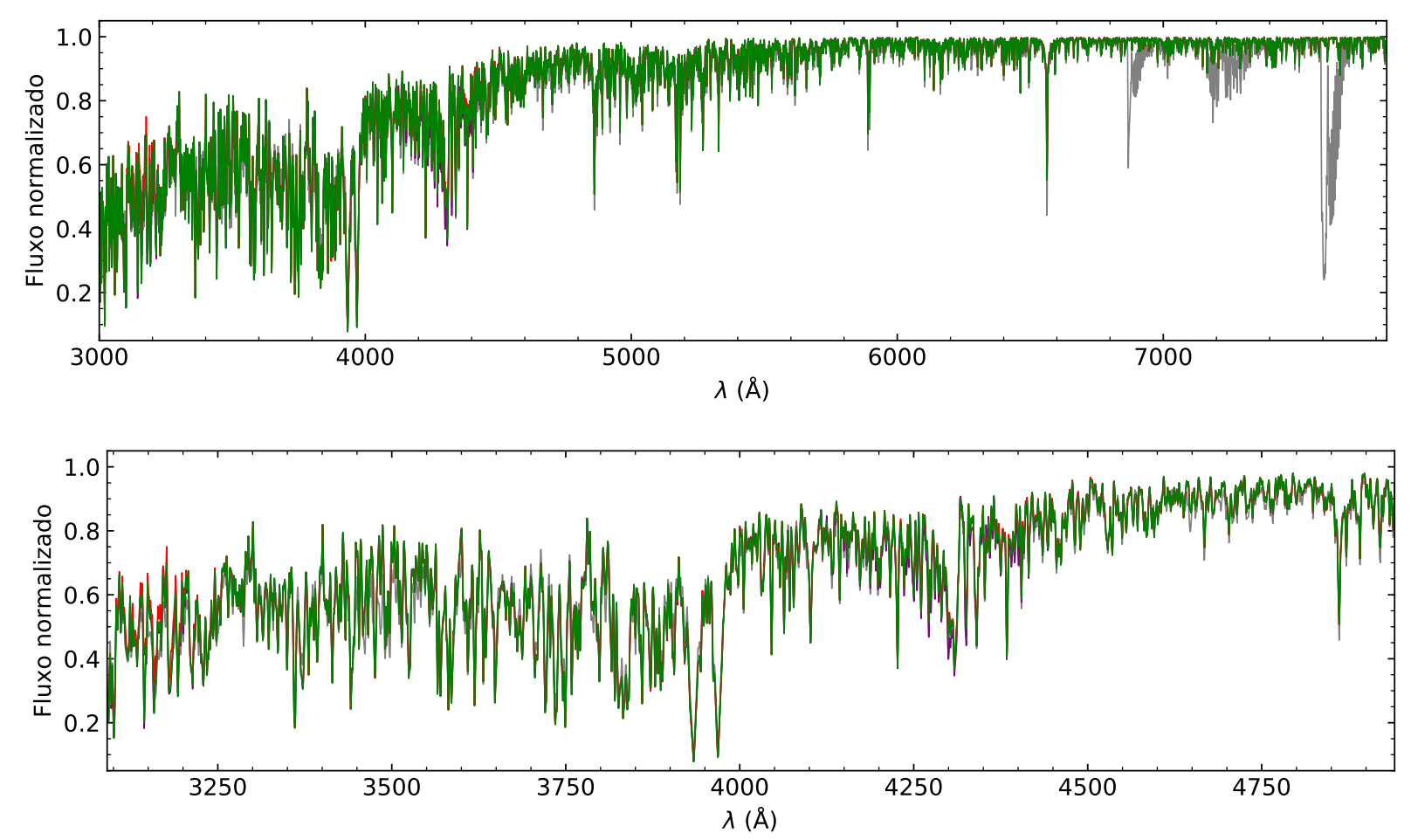

Figura 3.16: Espectros sintéticos das listas de opacidade da molécula CH "Kurucz" (roxo), "Jorgensen"(vermelho) e "Masseron" (verde), conforme Tabela 3.2, comparados com o espectro observado do Sol por Kurucz 2005b (cinza), de 3000 a $7840 \AA$ (painel superior) e de 3091 a $4941 \AA$ (painel inferior). 
A Figura 3.17 apresenta, sem convolução, os espectros sintéticos calculados com as listas de opacidade de $\mathrm{CH}$ em comparação ao espectro observado do Sol em três intervalos de $8 \AA$ em que a banda vibracional (0-0) de $\mathrm{CH}[\mathrm{A}-\mathrm{X}], \mathrm{CH}[\mathrm{B}-\mathrm{X}]$ e $\mathrm{CH}[\mathrm{C}-\mathrm{X}]$ estão destacadas em Wallace et al. (2011). Verificamos que o fato da lista "Jorgensen" não apresentar linhas na região mais azul do espectro se reflete na região da transição molecular [C-X] (painel inferior de 3.17). Na região relativa à transição [A-X], o espectro referente à lista "Kurucz" aparece mais metálico do que as outras e visualmente, em muitos casos, não representa tão bem o espectro solar. Com isto, e devido à modificação da lista "Kurucz" com Masseron et al. (2014) ser mais atual, escolhemos a lista de opacidades "Masseron".
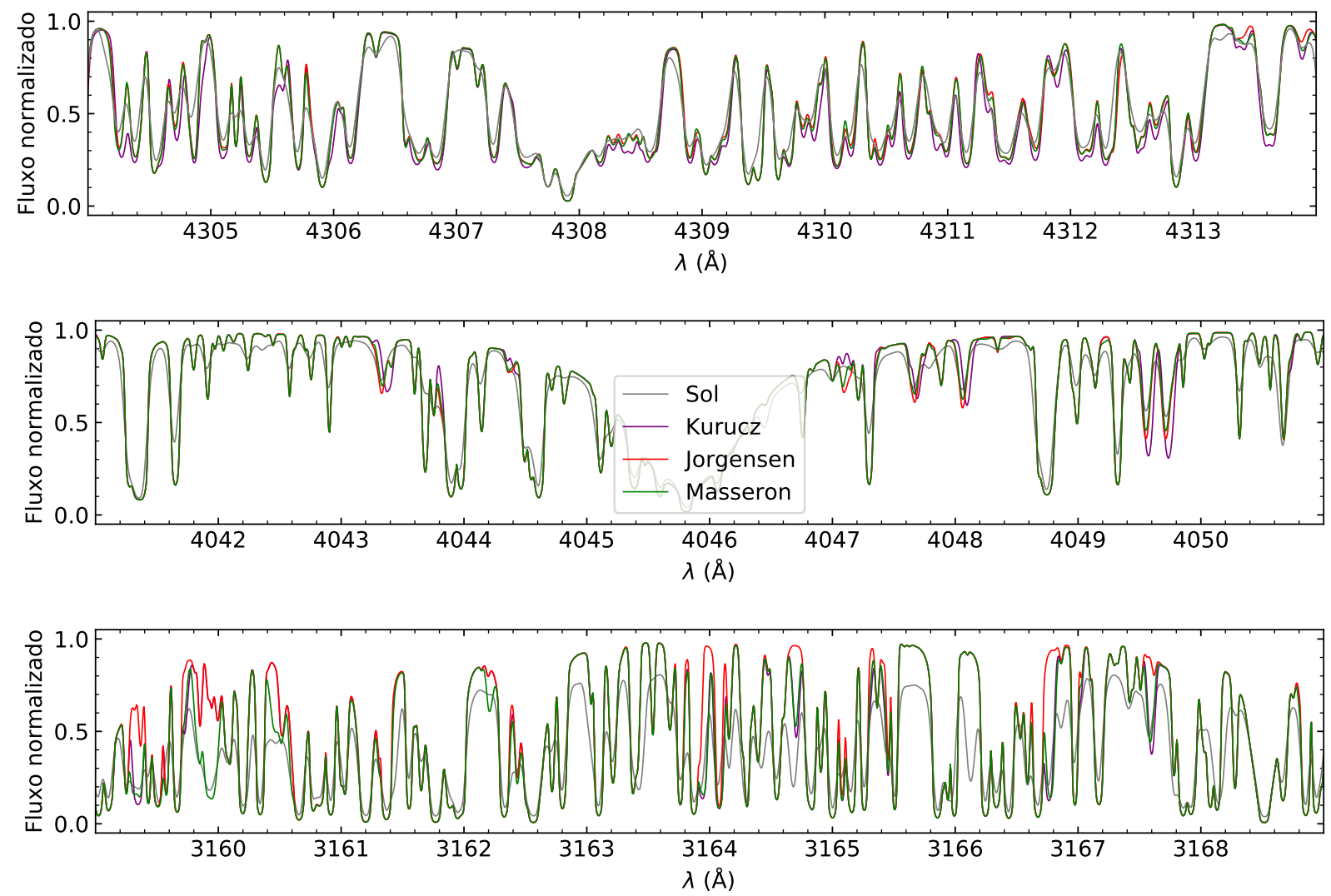

Figura 3.17: Painel de espectros sintéticos não convoluídos das listas de opacidade da molécula CH com "Kurucz" (roxo), "Jorgensen" (vermelho) e "Masseron" (verde), conforme Tabela 3.2, comparados com o espectro observado do Sol por Kurucz (2005b) (cinza), em regiões estreitas das transições moleculares [A-X] (cima), [B-X] (meio) e [C-X] (baixo), de 4305 a $4313 \AA$, 4042 a $4050 \AA$, e 3160 a $3168 \AA$, respectivamente. 


\subsubsection{Lista final de moléculas selecionadas}

Por fim, na Tabela 3.3 apresentamos a lista final de moléculas selecionadas, seu endereço eletrônico e nome de arquivo, cujas opacidades moleculares foram utilizadas para os cálculos da grade de espectros sintéticos deste trabalho.

Tabela 3.3 - Lista de espécies moléculares e respectivos arquivos usados neste trabalho.

\begin{tabular}{|c|c|c|}
\hline Molécula & Diretório & Arquivo \\
\hline $\mathrm{AlH}[\mathrm{A}-\mathrm{X}]$ & http://kurucz.harvard.edu/molecules/alh & alhax.asc \\
\hline $\mathrm{AlH}[\mathrm{B}-\mathrm{X}]$ & http://kurucz.harvard.edu/molecules/alh & alhxx.asc \\
\hline $\mathrm{AlO}$ & http://kurucz.harvard.edu/molecules/alo & alopatrascu.asc \\
\hline $\mathrm{C}_{2}[\mathrm{~A}-\mathrm{X}]$ & http://kurucz.harvard.edu/linelists/linesmol & c2ax.asc \\
\hline $\mathrm{C}_{2}[\mathrm{~B}-\mathrm{A}]$ & http://kurucz.harvard.edu/linelists/linesmol & c2ba.asc \\
\hline $\mathrm{C}_{2}[\mathrm{D}-\mathrm{A}]$ & http://kurucz.harvard.edu/molecules/c2 & c2dabrookek.asc \\
\hline $\mathrm{C}_{2}[\mathrm{E}-\mathrm{A}]$ & http://kurucz.harvard.edu/linelists/linesmol & c2ea.asc \\
\hline $\mathrm{CaH}$ & http://kurucz.harvard.edu/molecules/cah & cah.asc \\
\hline $\mathrm{CaO}$ & http://kurucz.harvard.edu/molecules/cao & caoyurchenko.asc \\
\hline $\mathrm{CH}$ & http://kurucz.harvard.edu/molecules/ch & chmasseron.asc \\
\hline $\mathrm{CN}[\mathrm{A}-\mathrm{X}]$ & http://kurucz.harvard.edu/linelists/linesmol & cnax.asc \\
\hline $\mathrm{CN}[\mathrm{B}-\mathrm{X}]$ & http://kurucz.harvard.edu/linelists/linesmol & cnbx.asc \\
\hline $\mathrm{CN}[\mathrm{X}-\mathrm{X}]$ & http://kurucz.harvard.edu/molecules/cn & cnxx12brooke.asc \\
\hline $\mathrm{CO}[\mathrm{A}-\mathrm{X}]$ & http://kurucz.harvard.edu/linelists/linesmol & coax.asc \\
\hline $\mathrm{CO}[\mathrm{X}-\mathrm{X}]$ & http://kurucz.harvard.edu/linelists/linesmol & coxx.asc \\
\hline $\mathrm{CrH}[\mathrm{A}-\mathrm{X}]$ & http://kurucz.harvard.edu/molecules/crh & crhaxbernath.asc \\
\hline $\mathrm{FeH}[\mathrm{F}-\mathrm{X}]$ & http://kurucz.harvard.edu/molecules/feh & fehfx.asc \\
\hline $\mathrm{H}_{2}$ & http://kurucz.harvard.edu/linelists/linesmol & h2.asc \\
\hline $\mathrm{MgH}$ & http://kurucz.harvard.edu/molecules/mgh & mgh.asc \\
\hline
\end{tabular}

Continua na próxima página... 
Tabela 3.3 - Continuação.

\begin{tabular}{|c|c|c|}
\hline Molécula & Diretório & Arquivo \\
\hline $\mathrm{MgO}$ & http://kurucz.harvard.edu/molecules/mgo & mgodaily.asc \\
\hline $\mathrm{NaH}$ & http://kurucz.harvard.edu/molecules/nah & nahrivlin.asc \\
\hline $\mathrm{NH}$ & http://kurucz.harvard.edu/linelists/linesmol & nh.asc \\
\hline $\mathrm{OH}$ & http://kurucz.harvard.edu/molecules/oh & ohupdate.asc \\
\hline $\mathrm{SiH}$ & http://kurucz.harvard.edu/linelists/linesmol & sihnew.asc \\
\hline $\mathrm{SiO}[\mathrm{A}-\mathrm{X}]$ & http://kurucz.harvard.edu/linelists/linesmol & sioax.asc \\
\hline $\mathrm{SiO}[\mathrm{E}-\mathrm{X}]$ & http://kurucz.harvard.edu/linelists/linesmol & sioex.asc \\
\hline $\mathrm{SiO}[\mathrm{X}-\mathrm{X}]$ & http://kurucz.harvard.edu/linelists/linesmol & sioxx.asc \\
\hline $\mathrm{TiH}$ & http://kurucz.harvard.edu/molecules/tih & tih.asc \\
\hline $\mathrm{TiO}$ & http://kurucz.harvard.edu/molecules/tio & tioschwenke.asc \\
\hline $\mathrm{VO}$ & http://kurucz.harvard.edu/molecules/vo & vo.asc \\
\hline
\end{tabular}

\subsubsection{Opacidades atômicas adotadas}

Em relação às opacidades atômicas, estudamos três listas de linhas disponíveis na literatura, à saber: Coelho $(2014)^{25}$, Castelli (2016) ${ }^{26}$ e Kurucz (2018). Este estudo tinha o objetivo de identificar qual das listas melhor representava o espectro observado do Sol e a possível dependência com comprimento de onda. Para isto, adotamos a abundância solar de Asplund et al. (2009) e as opacidades moleculares listadas na Tabela 3.2, conforme discutido nas Subseções 3.4.1 e 3.4.2, respectivamente.

Para que os espectros sejam comparáveis ponto a ponto, quando necessário, os espectros sintéticos e telúrico foram rebinados em relação aos espectros observacionais do Sol e Arcturus. Este processo foi realizado pelo método REBIN (Figura 3.1), do PyGLobsterS,

\footnotetext{
${ }^{25}$ Lista obtida por comunicação privada com a autora.

26 http://wwwuser.oats.inaf.it/castelli/linelists.html O ano se em parêntese no texto refere à data informada no website. Último acesso em: 08-08-2018.
} 
que interpola linearmente $e^{27}$ os espectros sintéticos para os mesmos elementos de comprimento de onda dos espectros observacionais.

Para avaliar a performance de cada lista, recorremos à métrica dada pela Equação 3.1:

$$
\mathrm{ADEV}=\frac{1}{N} \sum_{\lambda_{1}}^{\lambda_{2}}\left|\frac{f_{\text {sint }}\left(\lambda_{i}\right)-f_{\text {obs }}\left(\lambda_{i}\right)}{f_{\text {obs }}\left(\lambda_{i}\right)}\right|
$$

onde ADEV se refere a "Absolute Deviation" (Desvio Absoluto, em tradução livre), $f_{\text {sint }}\left(\lambda_{i}\right)$ e $f_{\text {obs }}\left(\lambda_{i}\right)$ são os valores de fluxo dos espectros sintético e observado, respectivamente, no $i$-ésimo elemento de comprimento de onda $\lambda$, e $N$ é a quantidade de elementos considerados em um dado intervalo de comprimento de onda $\Delta \lambda=\lambda_{2}-\lambda_{1}$. Desta forma, quando $f_{\text {sint }}\left(\lambda_{i}\right) \rightarrow f_{\text {obs }}\left(\lambda_{i}\right), \mathrm{ADEV} \rightarrow 0$. Assim, em um dado intervalo de comprimento de onda $\Delta \lambda$, um espectro observado é tão bem representado quanto menor for o ADEV. Este processo é realizado pelo método ADEV (Figura 3.1) do PyGLobsterS.

O painel superior da Figura 3.18 mostra os ospectros sintéticos do Sol calculados com cada uma das três listas em comparação aos espectros observados do Sol e telúrico, convoluídos para melhor visualização. O painel inferior de 3.18 apresenta a performance de cada lista. Nessa representação, os ADEVs foram calculados em intervalos de $\Delta \lambda=5 \AA$. Para mitigar o efeito das linhas telúricas, os elementos $\lambda_{i}$ correspondendo ao fluxo telúrico normalizado $f_{\text {telúrico }}\left(\lambda_{i}\right)<0,99$ foram desconsiderados. A Figura 3.19 apresenta os mesmos gráficos de 3.18 para Arcturus. As escolhas $f_{\text {telúrico }}\left(\lambda_{i}\right)<0,99$ e intervalo $\Delta \lambda=5 \AA$ para o cálculo dos ADEVs em 3.18 e 3.19 foram arbitrárias. De fato, neste cenário, para extrair a melhor performance ADEV possível, precisamos escolher a quantidade de contaminação do fluxo telúrico $f_{\text {telúrico }}$ permitida e o intervalo $\Delta \lambda$ que usaremos para avaliar as listas de linhas atômicas.

\footnotetext{
27 Método interp1d, do pacote ScIPy (Virtanen et al. 2020) no Python.
} 


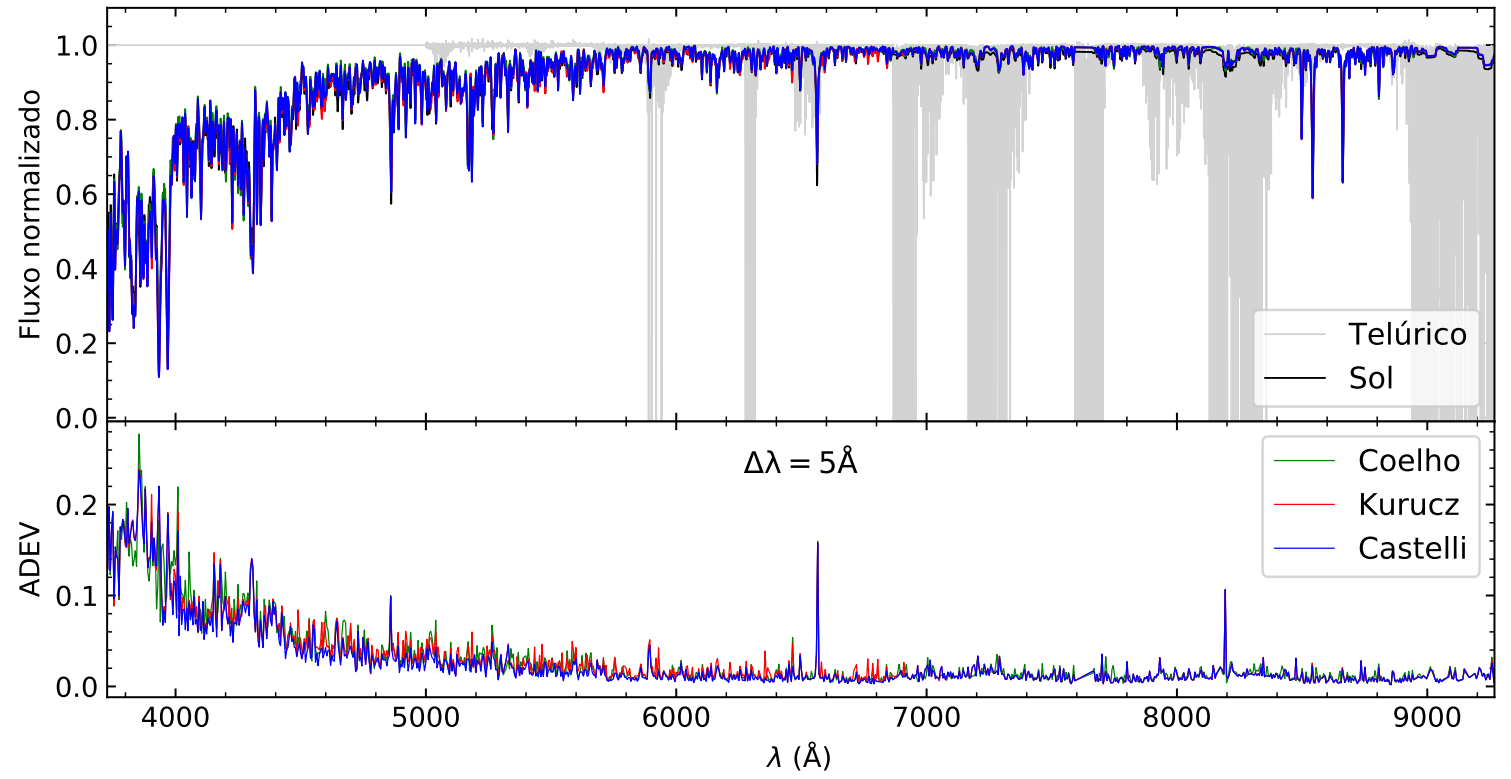

Figura 3.18: Painel superior: espectros sintéticos calculados com as listas de opacidade atômica Coelho (verde), Kurucz (vermelho) e Castelli (azul), comparados com o espectro observado do Sol por Kurucz (2005b) (preto), de 3727 a $9300 \AA$, sobrepostos ao espectro telúrico por Hinkle et al. (2000) (cinza claro). Painel inferior: ADEVs calculados em intervalos de $5 \AA$, referente a cada lista de opacidade atômica, no mesmo intervalo dos espectros.

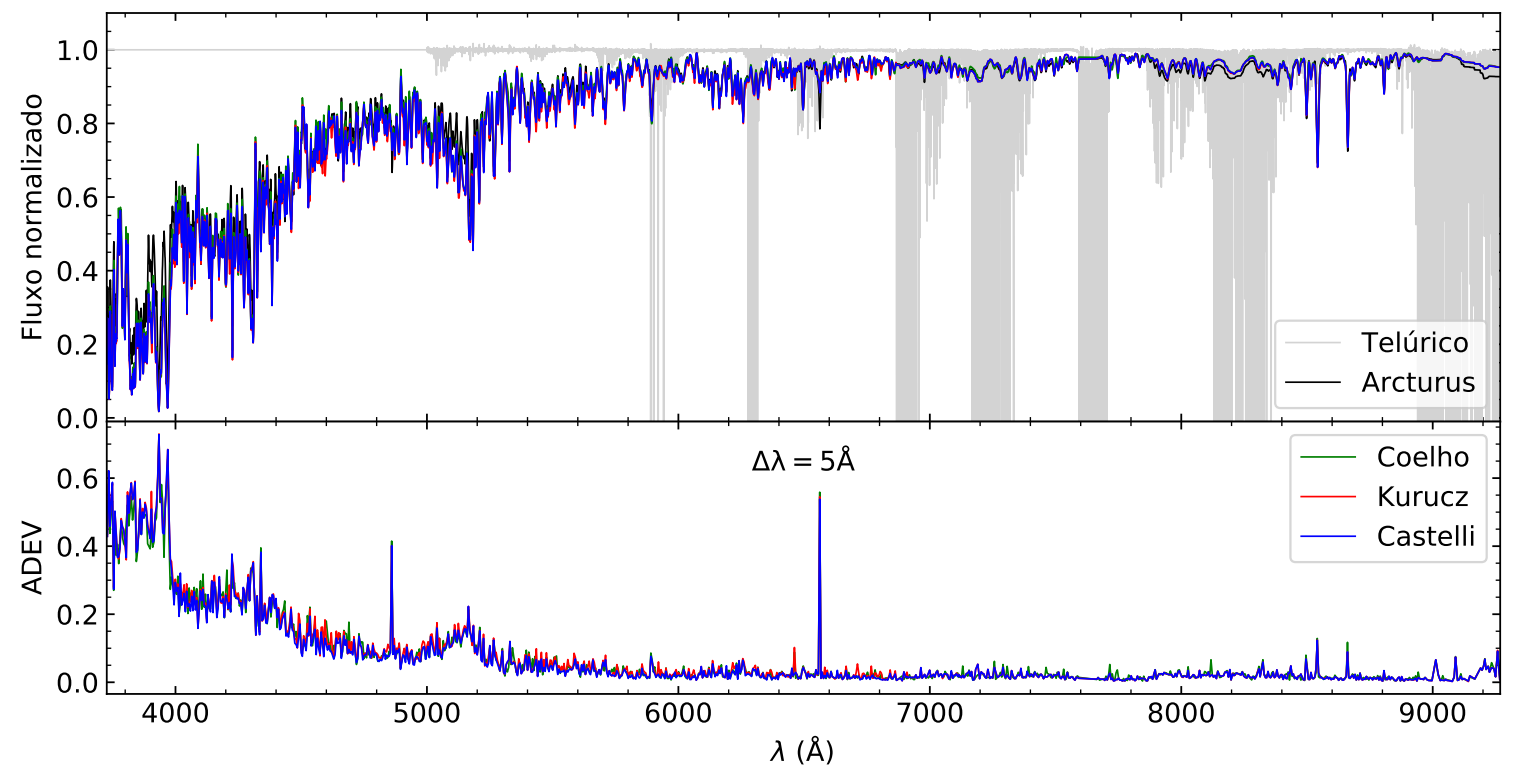

Figura 3.19: Painel superior: espectros sintéticos calculados com as listas de opacidade atômica Coelho (verde), Kurucz (vermelho) e Castelli (azul), comparados com o espectro observado de Arcturus por Hinkle et al. (2000) (preto), de 3727 a $9300 \AA$, sobrepostos ao espectro telúrico por Hinkle et al. (2000) em cinza claro. Painel inferior: ADEVs calculados em intervalos de $5 \AA$, referente a cada lista de opacidade atômica, no mesmo intervalo dos espectros. 
Para verificar o efeito da contaminação telúrica, calculamos o ADEV entre 3727 a $9300 \AA$ e a média do ADEV entre as três listas para $f_{\text {telúrico }}$ de 0,50 a 0,99 , para o Sol e Arcturus. Conforme mostra a Figura 3.20, a média dos valores ADEV entre as listas cai com a tolerância de contaminação telúrica adotada. Assim, daqui em diante, definimos que somente seriam levados em consideração nas análises os elementos em comprimento de onda dos espectros sintéticos e observados cujo fluxo normalizado telúrico fosse $f_{\text {telúrico }}\left(\lambda_{i}\right) \geq 0,99$.

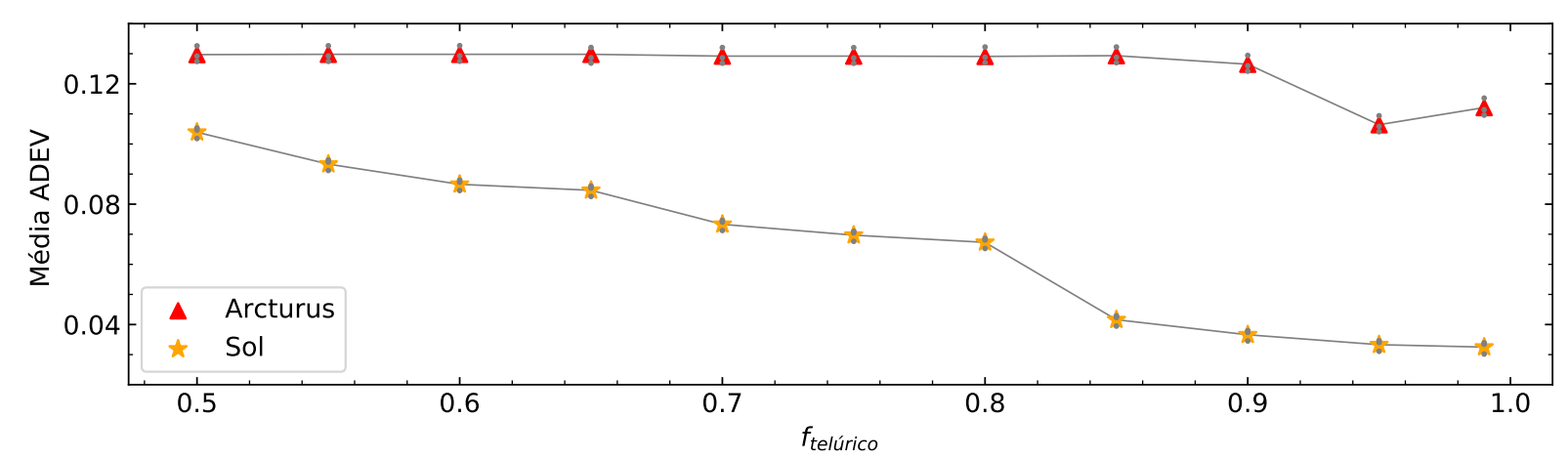

Figura 3.20: Média entre os valores ADEV das três listas de opacidade atômica (pontos cinza) em relação à tolerância de fluxo normalizado telúrico adotada, no intervalo de 3727 a $9300 \AA$, para o Sol (laranja) e Arcturus (vermelho).

Com relação a qual intervalo de comprimento de onda $\Delta \lambda$ adotar, foram calculados ADEV considerando diversos $\Delta \lambda$, de 0,1 a $10,0 \AA$. A Figura 3.21 ilustra a proporção de vezes que cada lista tem o menor $\mathrm{ADEV}$ em cada intervalo $\Delta \lambda$ testado. Os painéis superior e inferior mostram o resultado considerando os testes realizados com espectros do Sol e Arcturus, respectivamente. Nota-se que a quantidade de vezes que cada lista tem a melhor performance varia por intervalo $\Delta \lambda$ e por estrela, mas que de modo geral as listas que performam melhor são Castelli para o Sol e Coelho para Arcturus. 

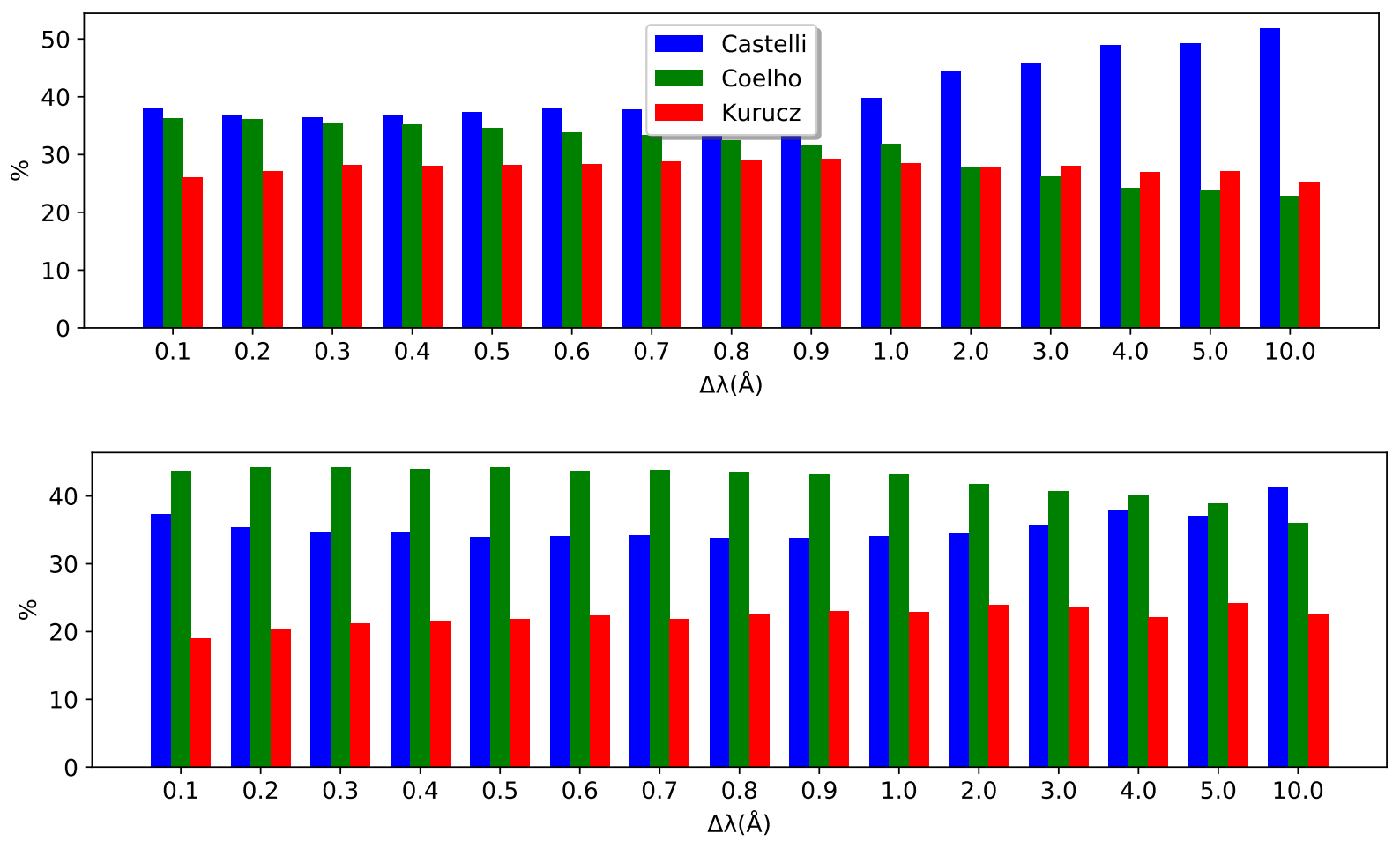

Figura 3.21: Proporção (\%) que cada lista obteve o menor ADEV em função do intervalo $\Delta \lambda$ (de 0.1 a $10.0 \AA$ ) considerado. Os painéis superior e inferior ilustram os resultados usando Sol e Arcturus como referência, respectivamente.

\subsubsection{Novas listas de opacidades atômicas}

Vimos na seção anterior que o ADEV dos espectros sintéticos, calculados com as listas de opacidade atômica de referência (Castelli, Coelho e Kurucz), produziu resultados diferentes quando aplicado para o Sol e Arcturus. As listas de linhas também tiveram performances distintas em cada intervalo $\Delta \lambda$ calculado ao longo de todo o comprimento de onda estudado.

Estas diferenças de performance das listas de referência entre as estrelas nos motivou a produzir e testar duas novas listas de opacidades atômicas, uma calibrada em relação ao Sol e outra em relação à Arcturus. Os resultados preliminares foram apresentados na XVI LARIM $^{28}$ em 2019 e publicados como artigo de conferência do mesmo evento (Branco e Coelho, 2020). Em cada caso, fixamos a tolerância de fluxo normalizado telúrico $f_{\text {norm }} \geq 0,99$

\footnotetext{
28 https://www.iau.org/science/meetings/past/regional/2311/
} 
e o ADEV foi calculado com auxílio do método ADEV do PYGLOBSTERS, adotando intervalos $\Delta \lambda=0,2 \AA$ e cobrindo todo o intervalo do espectro telúrico e observado de Arcturus, i.e., de 3727 a $9300 \AA$ A. Para cada $\Delta \lambda$, identificamos a melhor dentre as três listas de referência (menor ADEV) e as opacidades nesse intervalo foram adotadas nas novas listas. Fizemos testes com diversos intervalos $\Delta \lambda$, de 0,1 a $5 \AA$, e escolhemos $\Delta \lambda=0,2 \AA$ que nos pareceu um compromisso entre capturar detalhes das linhas sem criar muitos problemas com linhas migratórias, explicadas a seguir.

De posse das listas de opacidades atômicas calibradas em relação ao Sol e Arcturus, calculamos os espectros sintéticos das estrelas permutando cada lista com as abundâncias químicas usadas neste trabalho, isto é, G\&S98 (Grevesse e Sauval, 1998), Asplund+05 (Asplund et al. 2005) e Asplund+09 (Asplund et al., 2009) para o Sol e R\&P11 (Ramírez e Prieto, 2011) para Arcturus. Contudo, notamos que algumas linhas haviam "sumido", ou seja estavam presentes nos espectros sintéticos calculados com as listas de referências mas não nos sintéticos calculados com a nova lista. Esse efeito pode ser visualizado na Figura 3.22 com a diferença do espectro da nova lista em relação aos espectros das listas de referência e o espectro observado do Sol. Exceto o espectro calculado com a nova lista, os outros espectros sintéticos apresentam perfis de linha em relação ao espectro observado em torno do $\mathrm{Na}$ e uma linha característica de FeI, que ilustramos em 3.22 em laranja, indicado no espectro solar por Wallace et al. (2011). Isto nos indicou que provavelmente outras linhas poderiam ter sumido.

Abordamos essa questão comparando a nova lista com as três listas de referência nas regiões em comprimento de onda em que as linhas tinham desaparecido. Verificamos que o valor do comprimento de onda central das linhas desaparecidas diferia entre as listas e caia em intervalos de $\Delta \lambda$ diferentes. Quando havia mudança da melhor lista de opacidades atômicas de um $\Delta \lambda$ para outro, algumas linhas nunca eram selecionadas pois estavam sempre na lista não selecionada. Em outras palavras, o comprimento de onda central da linha "perdida" estava fora dos intervalos que tiveram a melhor performance. 


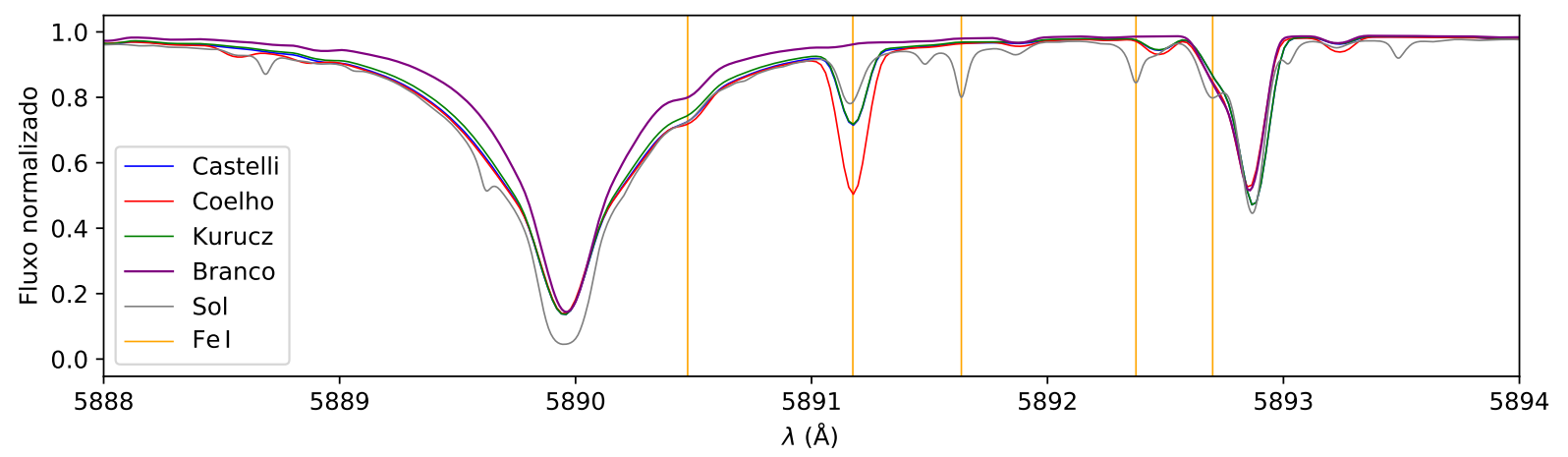

Figura 3.22: Diferença no perfil das linhas na região do Na entre os espectros sintéticos calculados com as listas de referência Castelli (azul), Coelho (vermelho) e Kurucz (verde) e com a nova lista Branco (roxo), em comparação ao espectro observado do Sol por Kurucz (2005b) (cinza claro), de 5888 a $5894 \AA$, com linhas características de Fe I indicadas pelas linhas verticais em laranja.

Um exemplo desta situação é apresentado na Tabela 3.4. A mudança da lista com menor ADEV calculado ocorre em $\lambda^{\prime}=5889,945 \AA$, de Coelho para Castelli. O comprimento de onda da linha de exemplo está centrado em $\lambda_{\text {coelho }}=5889,951 \AA$ para Coelho e em $\lambda_{\text {castelli }}=5889,939 \AA$ para Castelli, de modo que $\lambda_{\text {coelho }}>\lambda^{\prime}$ e $\lambda_{\text {castelli }}<\lambda^{\prime}$. Estas linhas não foram selecionadas devido a essa pequena diferença no comprimento de onda central, deixando-as em um $\Delta \lambda$ diferente cujo ADEV, por ocasião, não foi o menor/melhor. Assim, chamamos de "linhas migratórias" estas linhas de opacidade atômica que são "perdidas" ao longo da seleção e não foram adicionadas na nova lista de opacidades atômicas.

Tabela 3.4 - Exemplo de duas linhas de opacidade atômica das listas por Coelho (cima) e Castelli (baixo). O valor em "ID" refere-se ao código atribuído a um átomo da tabela periódica por R. Kuruct29.

\begin{tabular}{|c|c|c|c|c|c|c|c|c|c|c|c|c|}
\hline $\begin{array}{c}\lambda_{\text {central }} \\
(\AA)\end{array}$ & $\begin{array}{l}\log \\
g f\end{array}$ & ID & $\begin{array}{c}\mathbf{E}_{\mathbf{1}} \\
\left(\mathrm{cm}^{-1}\right)\end{array}$ & $\mathbf{J}_{1}$ & $\begin{array}{l}\text { Conf. } \\
\text { Elet.1 }\end{array}$ & $\begin{array}{c}\mathbf{E}_{2} \\
\left(\mathrm{~cm}^{-1}\right)\end{array}$ & $\mathbf{J}_{\mathbf{2}}$ & $\begin{array}{l}\text { Conf. } \\
\text { Elet.2 }\end{array}$ & $\begin{array}{l}\log \gamma \\
\text { (Rad.) }\end{array}$ & $\begin{array}{l}\log \gamma \\
\text { (Stark) }\end{array}$ & $\begin{array}{c}\log \gamma \\
\text { (VdWaals) }\end{array}$ & Ref. \\
\hline 5889,951 & 0,112 & 11.00 & 0,000 & 0,5 & $3 \mathrm{~s} 2 \mathrm{~S}$ & 16973,368 & 1,5 & $3 \mathrm{p} 2 \mathrm{P}$ & 7,80 & $-5,64$ & $-7,63$ & NIST \\
\hline 5889,939 & 0,108 & 11.00 & 0,000 & 0,5 & $2 \mathrm{p} 6.3 \mathrm{~s} 2 \mathrm{~S}$ & 16973,366 & 1,5 & $2 \mathrm{p} 6.3 \mathrm{p} 2 \mathrm{P}$ & 7,79 & $-5,64$ & $-7,63$ & KEP \\
\hline
\end{tabular}

\footnotetext{
29 http://kurucz.harvard.edu/atoms/periodic.dat
} 
Portanto, se fazia necessário uma segunda etapa na construção das novas listas para recuperar as linhas migratórias e incluí-las na lista final. Para tanto, a cada troca de $\Delta \lambda$ durante o calculo do ADEV, fixamos um novo intervalo $\delta \lambda=0,02 \AA$ centrado no comprimento de onda final de $\Delta \lambda$. Dentro desse pequeno intervalo $\delta \lambda$, analisamos todas as linhas da lista selecionada (i.e., a com menor ADEV) e procurando as linhas correspondentes na lista selecionada no $\Delta \lambda$ seguinte. Identificamos as linhas "migratórias" quando:

1. os comprimentos de onda centrais $\lambda_{\text {central }}$ são diferentes;

2. os códigos de identificação "ID" do número atômico e estágio de ionização são iguais;

3. o primeiro e segundo níveis de energia $\left(E_{1,2}\right)$ são iguais;

4. o primeiro e segundo números de impulso quântico angular total $\left(J_{1,2}\right)$ são iguais;

Os pares de linhas que atenderam aos critéricos citados foram classificados como pares de linhas migratórias. Uma das duas linhas precisava ser adicionada à nova lista. Para definir qual das duas linhas de cada par seria adicionada, analisamos as referências atribuídas a elas, conforme o arquivo "gfall.ref" 30 por R. Kurucz, que identifica as referências das linhas segundo seu formato, e adotamos o seguinte critério:

1. Se as duas linhas têm referência, adotamos a mais recente: entre "K13" e "K88", respectivamente em referência à "Kurucz 2013" e "Kurucz 1988", escolhemos a linha da referência "K13".

2. Quandouma das linhas não tinha referência, adotamos a linha com referência.

3. No caso das linhas não terem referência, demos preferência para adicionar a linha na lista de Coelho, Castelli ou Kurucz, nesta ordem.

4. Houve casos de referências iguais para as duas linhas, e comprimentos de onda (ou outro parâmetro) um pouco diferente. Nesse caso demos preferência para adicionar a linha na lista de Coelho, Castelli ou Kurucz, nesta ordem. Exceto quando a referência era de algum artigo do Kurucz, nesse caso usamos Kurucz preferencialmente.

\footnotetext{
${ }^{30}$ As referências (e.g., coluna "Ref." da Tabela 3.4 são indexadas por R. Kurucz em seu website http://kurucz.harvard. edu/linelists/gfnew/gfall.ref
} 
Essa etapa de correção pelas linhas migratórias foi realizada apenas na lista calibrada ao espectro solar, que é que utilizamos no cálculo da grade. De posse da segunda versão da nova lista de opacidades atômicas, recalculamos os espectros sintéticos com os modelos de atmosfera solar e reavaliamos o ADEV em cada caso. A Tabela 3.5 compila os valores ADEV dos espectros sintéticos calculados com as listas de opacidade de referência e as listas calibradas que construímos neste trabalho. Verificamos quantitativamente que a performance da segunda versão melhorou em relação à primeira, após o tratamento de dados das "linhas migratórias". Por fim, na Figura 3.23, reapresentamos o gráfico de 3.22 com o espectro calculado com a nova lista após o estudo das linhas migratórias, ilustrando a correção no perfil das linhas em tordo do Na e Fe I em relação ao perfil das linhas dos outros espectros.

Tabela 3.5 - Comparação entre os ADEVs dos espectros sintéticos calculados com a primeira ("Sol v1") e segunda ("Sol v2") versões da nova lista de opacidade atômica calibradas com o Sol e a calibrada com Arcturus, e com as listas de referência por Castelli, Coelho e Kurucz, para as abundâncias químicas G\&S98 (Grevesse e Sauval, 1998), Asplund+05 (Asplund et al., 2005) e Asplund+09 (Asplund et al., 2009) para o Sol e R\&P11 (Ramírez e Prieto, 2011) para Arcturus.

\begin{tabular}{|c|c|c|c|c|c|c|c|}
\hline \multirow[b]{2}{*}{ Estrela } & \multirow[b]{2}{*}{$\begin{array}{c}\text { Abundância } \\
\text { Química }\end{array}$} & \multicolumn{6}{|c|}{ Lista de opacidade atômica } \\
\hline & & Castelli & Coelho & Kurucz & $\begin{array}{c}\text { Branco } \\
\text { (Ref. Arcturus) }\end{array}$ & $\begin{array}{c}\text { Branco } \\
\text { (Ref. Sol v1) }\end{array}$ & $\begin{array}{c}\text { Branco } \\
\text { (Ref. Sol v2) } \\
\end{array}$ \\
\hline \multirow{3}{*}{ Sol } & G\&S98 & 0,03013 & 0,03364 & 0,03354 & 0,03012 & 0,02728 & 0,02680 \\
\hline & Asplund +05 & 0,03022 & 0,03430 & 0,03339 & 0,03153 & 0,02885 & 0,02837 \\
\hline & Asplund+09 & 0,03020 & 0,03392 & 0,03345 & 0,03097 & 0,02805 & 0,02757 \\
\hline Arcturus & R\&P11 & 0,10958 & 0,11140 & 0,11525 & 0.10656 & 0,10601 & 0,10465 \\
\hline
\end{tabular}

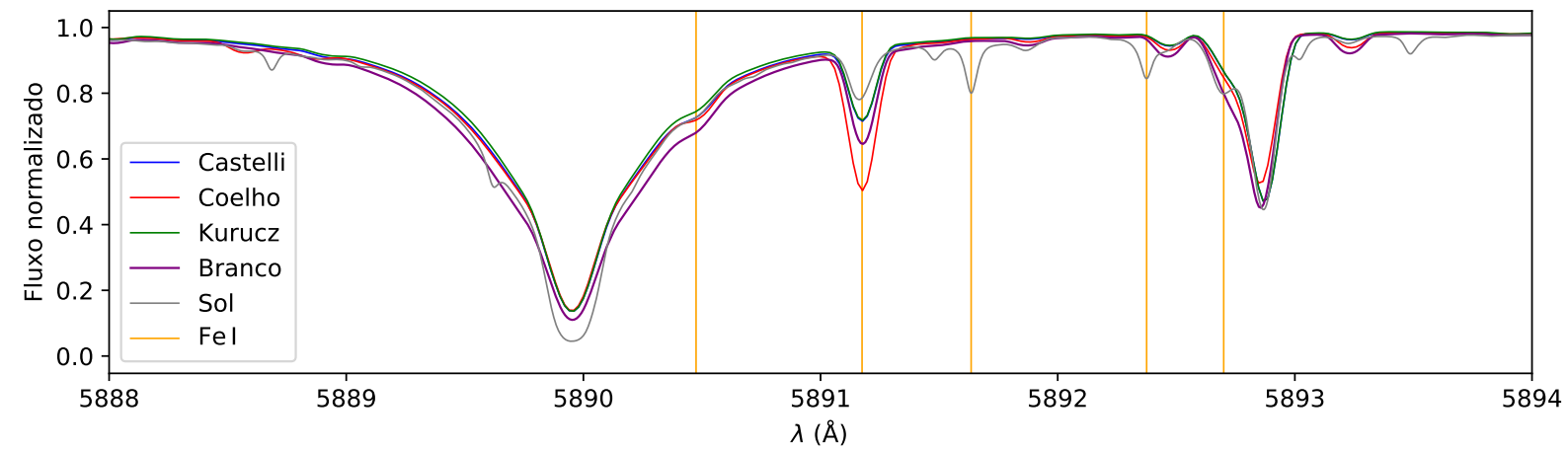

Figura 3.23: Reapresentação da Figura 3.22 com a espectro calculado com a nova lista "Branco" (roxo) corrigida. 
Capítulo 4

\section{A Grade de Espectros Sintéticos}

No capítulo anterior definimos os ingredientes da síntese espectral estelar e nossas análises resultaram em duas listas de opacidades atômicas que melhoraram a representação dos espectros observados do Sol e Arcturus. Com esses ingredientes definidos, podemos calcular uma grade de espectros sintéticos a serem usados em estudos de espectros integrados de aglomerados globulares.

Na Seção 4.1 apresentamos os parâmetros de entrada para os cálculos. Na Seção 4.3 definimos a cobertura da grade em termos de $T_{\text {eff }}, \log g$ e $[\mathrm{Fe} / \mathrm{H}]$. Neste trabalho adotamos dois padrões de abundância química, para representar duas gerações de estrelas nos AGs, discutidos e definidos na Seção 4.2. Por fim, na Seção 4.4 concluímos com uma discussão sobre os espectros sintéticos calculados da grade.

\subsection{Ingredientes adotados e características gerais}

Para a construção dos espectros da grade, usamos os programas ATLAS12 e SYNTHE para o cálculo de modelos de atmosfera (Seção 3.1) e espectros sintéticos (Seção 3.2), respectivamente executados através dos módulos PyATLAS e PySYNTHE do PyGlobsterS (Seção 3.3). Fixamos os seguintes parâmetros de entrada para o cálculo dos espectros conforme listados abaixo:

- Resolução amostral: $R_{\lambda}=300000$;

- Resolução espectral: $R_{\mathrm{LSF}}=150000$ (Gaussiana); 
- Intervalo em comprimento de onda de 3727 a $9300 \AA$;

- Abundância solar de referêncią? Asplund et al. (2009);

- Opacidades moleculares: conforme Seção 3.4 .2 ,

- Opacidades atômicas: nova lista de linhas calibrada em relação ao Sol (Seção 3.4.4).

- Cálculo do espectro de fluxo, com comprimentos de onda no ar.

A partir dos pares de parâmetros $T_{\text {eff }}$ e $\log g$, Martins et al. (2019) calcularam a correspondência destes pares aos modelos de atmosfera estelar por Coelho (2014). Contudo, para estrelas com $T_{\text {eff }}<4000(\mathrm{~K})$ foram usados modelos do MARCS (Gustafsson et al., 2008). Para cada AG deste trabalho, usamos como modelos de atmosfera de referência os modelos de Coelho (2014) que correspondiam aos pares de parâmetros $T_{\text {eff }}$ e $\log g$ em Martins et al. (2019). Para os pares relativos ao MARCS, usamos os modelos calculados em Coelho et al. (2011) e, quando necessário, os modelos de atmosfera calculados neste trabalho para completar a grade. Todos os modelos calculados aqui atenderam ao critério de convergência discutido em 3.3.1, com velocidade de microturbulência $V_{\text {turb }}=2 \mathrm{~km} / \mathrm{s}$, profundidade de mistura $=1,25$ e profundidade ótica Rosseland $\tau_{\text {Ross }}=-6.875$, sem overshooting.

\subsection{Padrões de abundância química}

Além dos pares de parâmetros $T_{\text {eff }}$ e $\log g$, a grade é fundamentalmente dependente do padrão de abundâncias químicas considerado nos modelos de atmosfera estelares. Como estamos interessados em estudar como as variações químicas afetam o espectro integrado dos aglomerados globulares, neste trabalho adotamos duas gerações de estrelas:

- A primeira geração é constituída de uma mistura química com elementos $\alpha$ enriquecidos $\left([\alpha / \mathrm{Fe}]=0.4\right.$, característico de estrelas do halo galáctic $\left.{ }^{2}\right)$, outros metais adotam o padrão solar (Asplund et al. 2009) escalonados para a abundância de ferro conforme Tabela 4.2 , fração de massa de Helio $\mathrm{Y}=0.256$;

\footnotetext{
${ }^{1}$ Para os AGs, o padrão de abundâncias químicas de cada caso é escalonado em função da metalicidade do AG (veja Seção 4.2 para mais detalhes).

2 Neste trabalho, foram considerados elementos $\alpha$ : O, Ne, Mg, Si, S, Ca e Ti.
} 
- A segunda geração considera a variação dos elementos químicos anticorrelacionados CN-ONa e o enriquecimento de Hélio, em relação à primeira geração, também adotado em Coelho et al. (2011):

$\mathrm{Y}=0.300 ; \Delta(\mathrm{C}, \mathrm{N}, \mathrm{O}, \mathrm{Na})=\Delta(-0.30,+1.20,-0.45,+0.60) \operatorname{dex}$.

A Tabela 4.1 apresenta os valores dos padrões químicos adotados das misturas químicas que representam a primeira e segunda gerações de cada aglomerado. Os parâmetros de entrada no ATLAS12 sobre as abundâncias químicas são dados em diferentes unidades: $[\mathbf{F e} / \mathbf{H}]$ (a) e $[\alpha / \mathbf{F e}]$ (b) são abundâncias relativas ao padrão solar (conforme definições apresentadas na Introdução); Y (b) é a fração de massa normalizada de He; H (c) e He (d) são as frações da quantidade de $\mathrm{H}$ e He, respectivamente; e as abundâncias de $\mathbf{C}, \mathbf{N}, \mathbf{O}$ e $\mathrm{Na}$ são aquelas relativas ao padrão solar adotado neste trabalho, na escala "A(Kurucz)" ${ }^{3}$, compatível com o ATLAS12.

Tabela 4.1 - Padrões de abundâncias químicas para NGC 1904, NGC 0104 e NGC 5927.

\begin{tabular}{|c|c|c|c|c|c|c|c|c|c|c|c|}
\hline Geração & $\mathbf{N G C}$ & {$[\mathbf{F e} / \mathbf{H}]^{(\mathrm{a})}$} & {$[\alpha / \mathbf{F e}]^{(\mathrm{a})}$} & $\mathbf{Y}^{(\mathrm{b})}$ & $\mathbf{H}^{(\mathrm{c})}$ & $\mathbf{H e}^{(\mathrm{c})}$ & $\mathbf{C}^{(\mathrm{d})}$ & $\mathbf{N}^{(\mathrm{d})}$ & $\mathbf{O}^{(\mathrm{d})}$ & $\mathbf{N a}^{(\mathrm{d})}$ \\
\hline $1^{\mathrm{a}}$ & 1904 & $-1,579$ & 0,4 & 0,256 & 0.92015 & 0.07980 & -3.61 & -4.21 & -2.95 & -5.80 \\
\hline $2^{\mathrm{a}}$ & 1904 & $-1,579$ & 0,4 & 0,300 & 0.90246 & 0.09749 & -3.91 & -3.01 & -3.40 & -5.20 \\
\hline $1^{\mathrm{a}}$ & 0104 & $-0,768$ & 0,4 & 0,256 & 0.91951 & 0.08017 & -3.61 & -4.21 & -2.95 & -5.80 \\
\hline $2^{\mathrm{a}}$ & 0104 & $-0,768$ & 0,4 & 0,300 & 0.90174 & 0.09793 & -3.91 & -3.01 & -3.40 & -5.20 \\
\hline $1^{\mathrm{a}}$ & 5927 & $-0,47$ & 0,4 & 0,256 & 0.91877 & 0.08059 & -3.61 & -4.21 & -2.95 & -5.80 \\
\hline $2^{\mathrm{a}}$ & 5927 & $-0,47$ & 0,4 & 0,300 & 0.90090 & 0.09844 & -3.91 & -3.01 & -3.40 & -5.20 \\
\hline
\end{tabular}

\footnotetext{
${ }^{3}$ Ver nota de rodapé 9 da Subseção 3.3 .1
} 


\subsection{Cobertura da grade em $T_{\text {eff }}, \log g$ e $[\mathrm{Fe} / \mathrm{H}]$}

Como apresentado na Seção 2.3, escolhemos três aglomerados globulares com metalicidades distintas para testar o trabalho, conforme a Tabela 4.2. Demos preferência para determinações recentes de $[\mathrm{Fe} / \mathrm{H}]$, listadas na quarta coluna da tabela. Assim, com as metalicidades e os pares de parâmetros $T_{\text {eff }}$ e $\log g$ para os aglomerados, calculamos os modelos de atmosfera estelar para cada um dos padrões de abundância química, representantes da primeira e segunda gerações de estrelas de cada AG. Na Figura 4.1, reapresentamos a Figura 2.7, sobrepondo o DHR da grade de modelos atmosféricos estelares calculados neste trabalhq ${ }^{4}$ ao DHR estimado por Martins et al. (2019) para NGC 1904, NGC 0104 e NGC 5927.

Tabela 4.2 - Aglomerados estudados e algumas medidas de metalicidade relatadas na literatura: (a) Harris (1996) 10 $10^{\mathrm{a}}$ Ed. (usado por Usher et al. 2017); (b) Harris (1996) (usado por Martins et al. 2019); (c) Carretta et al. (2009a); (d) Mura-Guzmán et al. (2018).

\begin{tabular}{|c|c|c|c|}
\hline NGC & \multicolumn{3}{|c|}{$[\mathrm{Fe} / \mathrm{H}]$} \\
\hline 1904 & $-1,60^{\text {(a) }}$ & $-1,55^{(\mathrm{b})}$ & $-1.579 \pm 0,011^{(\mathrm{c})}$ \\
\hline 0104 & $-0,72^{\text {(a) }}$ & $-0,76^{(\mathrm{b})}$ & $-0,768 \pm 0,016^{(\mathrm{c})}$ \\
\hline 5927 & $-0,49$ (a) & $-0,37^{(b)}$ & $-0,47 \pm 0,02^{(\mathrm{d})}$ \\
\hline
\end{tabular}
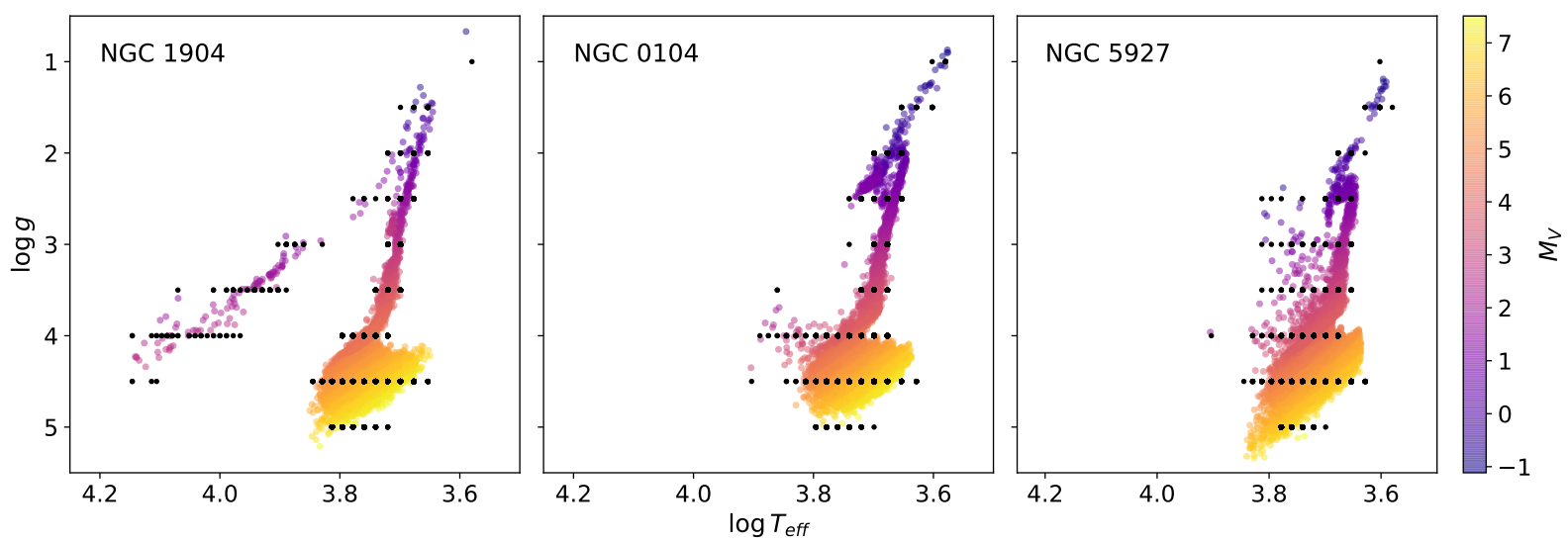

Figura 4.1: Sobreposição entre o DHR estimado por Martins et al. (2019) a partir das observações (pontos coloridos) e o DHR da grade de modelos atmosféricos calculados neste trabalho (preto) para NGC 1904 (esquerda), NGC 0104 (meio) e NGC 5927 (direita).

\footnotetext{
${ }^{4}$ As Tabelas B.1 B.2 B.3 no Apêndice, apresentam os valores de todos os pares dos parâmetros $T_{\text {eff }}$ e $\log g$ relativos a NGC 1904, NGC 0104 e NGC 5927, respectivamente.
} 


\subsection{Espectros da grade}

Para concluir esta etapa, foi calculada uma grade de 376 espectros sintéticos, conforme sintetiza a Tabela 4.3 com a quantidade de espectros calculados para cada aglomerado globular. A cobertura em $T_{\text {eff }}$ e $\log g$ são dadas na Seção 4.3, com os padrões de abundância química definidos na Seção 4.2. Estes espectros cobrem o diagrama-HR de forma diferente para cada abundância de Ferro adotada, buscando representar o CMD dos aglomerados globulares (vide Figura 4.1). Com isto, construímos três subgrades de espectros sintéticos, uma para cada metalicidade representantes dos três AGs que usamos como referência.

Tabela 4.3 - Quantidade de espectros sintéticos calculados por aglomerado, com a cobertura de parâmetros atmosféricos e padrões de abundância química definidos neste trabalho.

\begin{tabular}{|c|c||c|c||c|c|}
\hline NGC & $\#$ & NGC & $\#$ & NGC & $\#$ \\
\hline 1904 & 104 & 0104 & 152 & 5927 & 120 \\
\hline
\end{tabular}

Na Figura 4.2, no painel superior, mostramos os espectros sintéticos de dois modelos de atmosfera estelar de estrelas de NGC 0104 um representante da primeira (" $\alpha+$ ") e outro da segunda ( " $\alpha+, \mathrm{He}+, \Delta \mathrm{CNONa")} \mathrm{geração} \mathrm{de} \mathrm{estrelas} \mathrm{deste} \mathrm{aglomerado,} \mathrm{comparados} \mathrm{com}$ o espectro observado do Sol. Para comparar com o espectro solar, escolhemos o par de parâmetros atmosféricos mais próximo da grade de espectro de NGC 0104: $T_{\text {eff }}=5750 \mathrm{~K}$ e $\log g=4,5$. No painel inferior de 4.2 apresenta o mesmo tipo de comparação em relação Arcturus, com $T_{\text {eff }}=4250 \mathrm{~K}$ e $\log g=1,5$. Em ambos os casos, os espectros sintéticos foram calculados com resolução amostral e espectral relativas às das observações, i.e., $R_{\lambda}$ $=600000$ e $R_{\mathrm{LSF}}=300000$ em relação ao Sol e $R_{\lambda}=300000$ e $R_{\mathrm{LSF}}=150000$ à Arcturus. Todos os espectros em 4.2 foram degradados para melhor visualização com sigma $=150$. Nos gráficos inferiores de cada painel ilustramos o resíduo entre os espectros sintético: 5 , A partir dos resíduos, verificamos que, com parâmetros atmosféricos próximos aos do Sol, as bandas de $\mathrm{CN}[\mathrm{A}-\mathrm{X}]$ são as mais afetadas no espectro sintético estelar, enquanto que em relação a Arcturus, tanto as linhas $\mathrm{CN}[\mathrm{A}-\mathrm{X}]$ quanto $\mathrm{CN}[\mathrm{B}-\mathrm{X}]$ são afetadas, assim como a região do Na.

\footnotetext{
${ }^{5} \mathrm{O}$ resíduo é a diferença entre o fluxo do espectro sintético estelar que representa a primeira geração $F_{\lambda, 1}$ e o fluxo do espectro sintético estelar que representa a segunda geração $F_{\lambda, 2}$, i.e., Resíduos $=F_{\lambda, 1}-F_{\lambda, 2}$.
} 

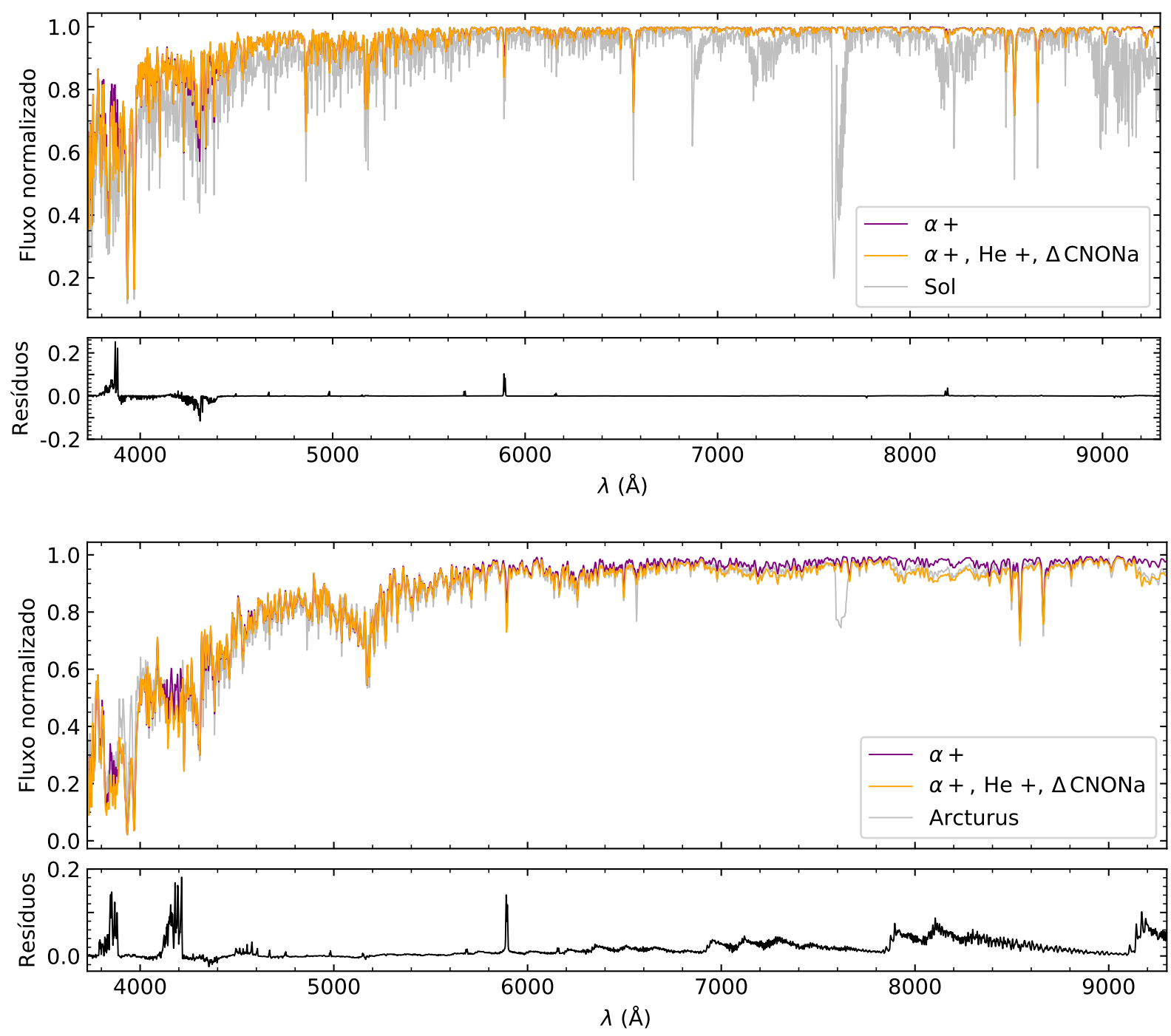

Figura 4.2: Gráfico superior de cada painel: Espectros sintéticos representantes da primeira (roxo) e segunda (laranja) geração de estrelas de NGC 0104 em comparação ao espectro observado do Sol por Kurucz (2005b) (painel superior), com $T_{\text {eff }}=5750 \mathrm{~K}$ e $\log g=4,5$, e Arcturus por Hinkle et al. (2000) (painel inferior), com $T_{\text {eff }}=4250 \mathrm{~K}$ e $\log g=1,5$, ambos em cinza. Gráfico inferior de cada painel: resíduos entre os espectros sintéticos (detalhes no texto).

Na Figura 4.3, ilustramos os mesmos espectros da Figura 4.2, mas restringindo o gráfico a regiões sensíves à molécula de $\mathrm{CN}$ e às linhas atômicas de $\mathrm{O}$ e $\mathrm{Na}$, em semelhança à Coelho et al. (2011). Ao passo que o impacto das variações no espectro sintético quase não aparece na região do $\mathrm{O}$ (painel do meio), nas regiões sensíveis à $\mathrm{CN}$ e Na (paineis de cima e baixo, respectivamente) as diferenças podem ser visualmente melhor identificadas. 

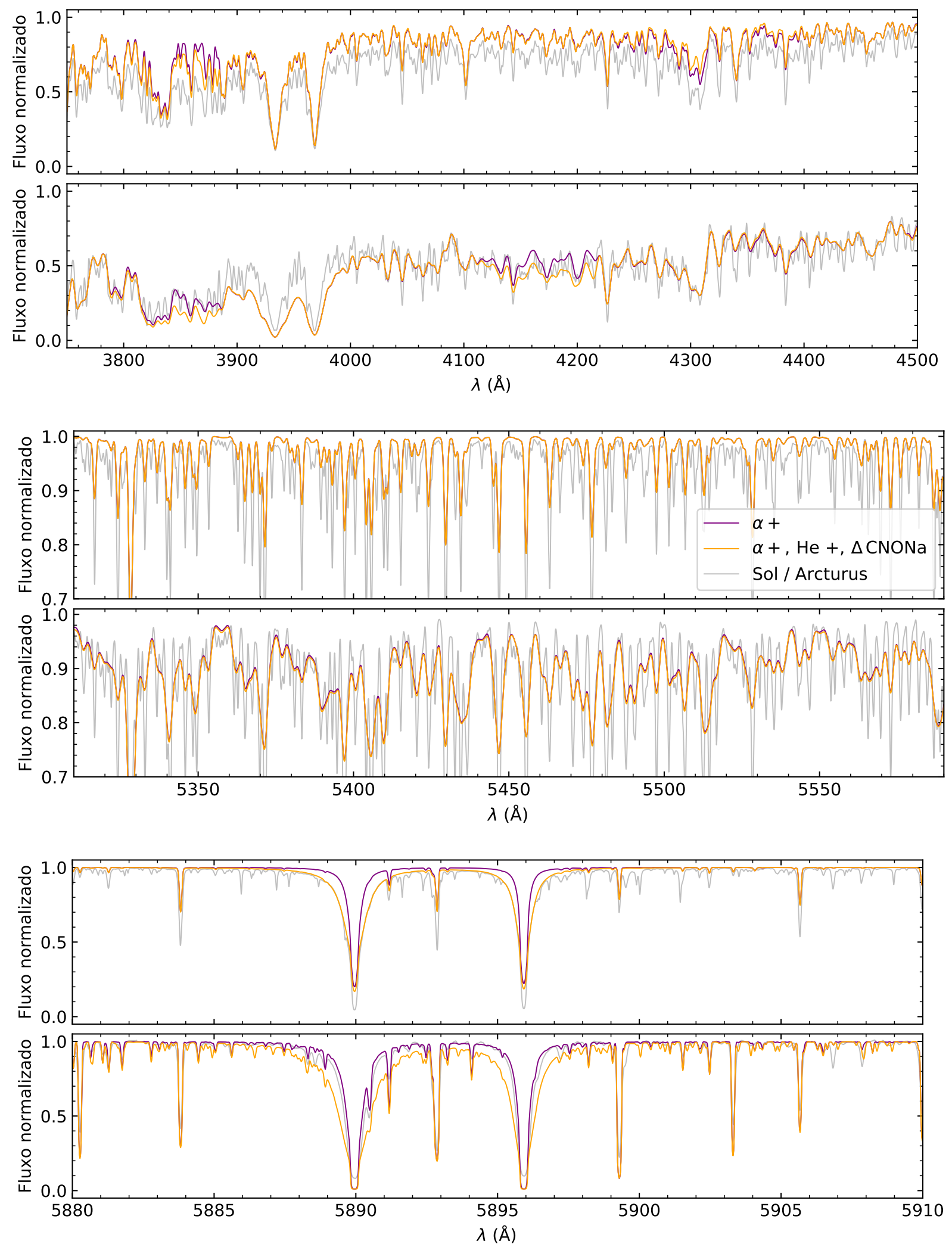

Figura 4.3: Espectros sintéticos estelares da primeira (roxo) e segunda (laranja) gerações de estrelas de NGC 0104 nas regiões de CN (painel superior), O (painel do meio) e Na (painel inferior), em comparação aos espectros observados do Sol (gráfico superior de cada painel) por Kurucz (2005b) e Arcturus (gráfico inferior de cada painel) por Hinkle et al. (2000) (ambos em cinza). 
Por fim, a Figura 4.4 apresenta os espectros sintéticos estelares calculados com os valores mínimos e máximos do espaço de parâmetross $T_{\text {eff }}$ e $\log g$ da grade para NGC 1904 NGC 0104 e NGC 5927 no intervalo no qual calculamos a nova lista de opacidades atômicas, i.e., de 3727 a $9300 \AA$. Em cada linha de 4.4, os paineis apresentam a metalicidade e o par $T_{\text {eff }}$ e $\log g$ com que os espectros sintéticos foram calculados, relativos às de cada AG e mostra o efeito para cada hipótese de mistura química que estudamos. Enquanto notamos visualmente que a diferença entre as duas misturas aumenta com a metalicidade para os espectros sintéticos das estrelas de baixa temperatura efetiva e gravidade superficial (paineis da primeira coluna), o mesmo efeito não é possível ser verificado para os espectros sintéticos da estrelas mais quentes e maior gravidade superficial (paineis da segunda coluna). De todo modo, agora, podemos usar a grade no espaço de parâmetros $\{[\mathrm{Fe} / \mathrm{H}]$,

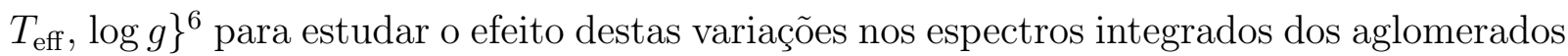
globulares. Os detalhes destes cálculos e suas aplicações são discutidos no próximo capítulo.

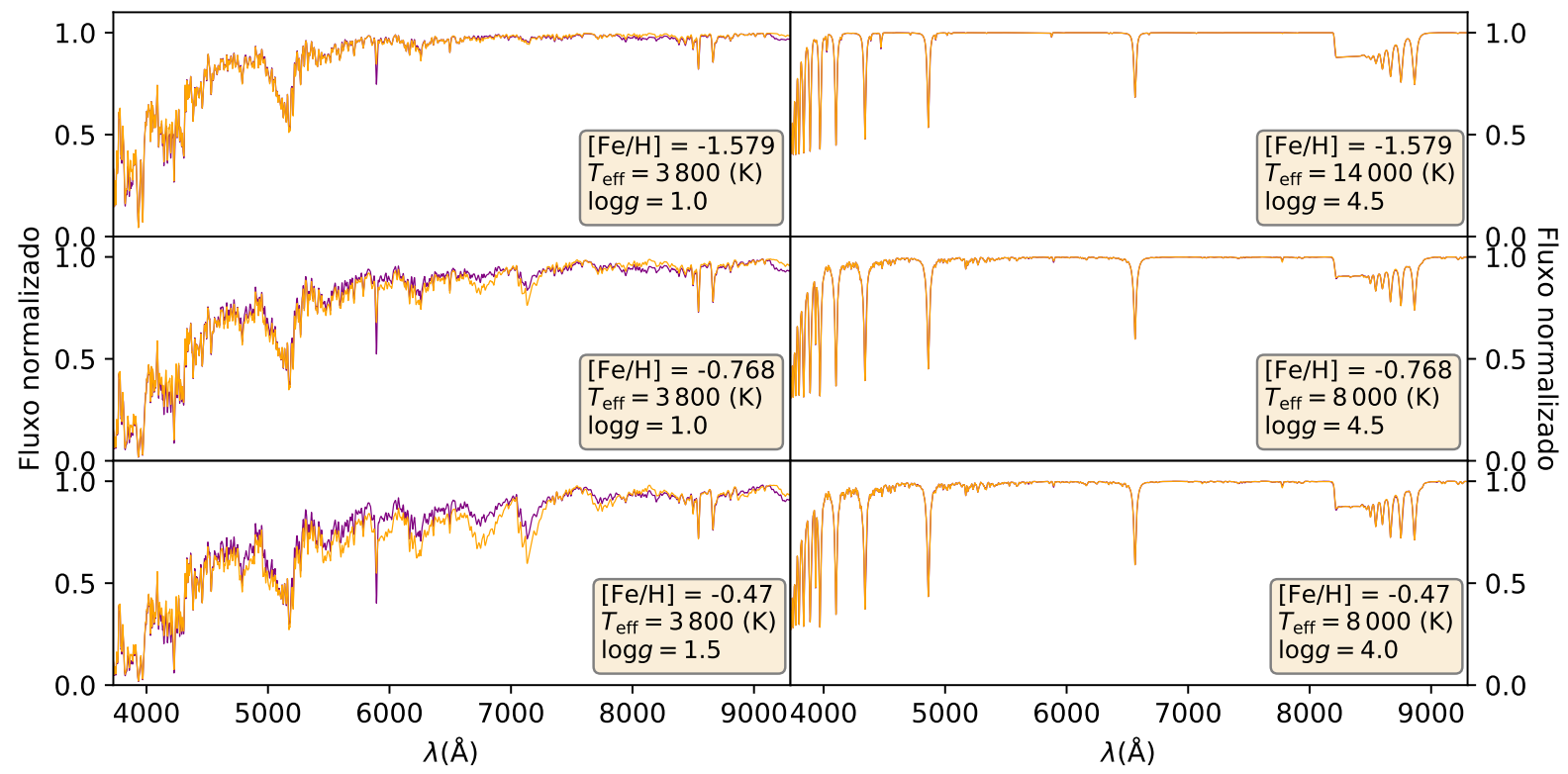

Figura 4.4: Espectros sintéticos estelares representantes da primeira (roxo) e segunda (laranja) geração de estrelas de NGC 1904 (paineis superiores), NGC 0104 (paineis do meio) e NGC 5927 (painel inferior). Cada linha apresenta os espectros sintéticos calculados com o valor mínimo e máximo do par de parâmetros $T_{\text {eff }}$ e $\log g$ da grade para cada AG e suas metalicidades, de 3727 a $9300 \AA$.

\footnotetext{
${ }^{6}$ Veja Tabelas B.2 B.1 B.3 para consultar a quantidade de estrelas consideradas com os pares $\left\{T_{\text {eff }}, \log g\right\}$ de cada AG.
} 
Capítulo 5

\section{Aplicação a Aglomerados Globulares Galácticos}

Do capítulo anterior, vimos que variações nas abundâncias químicas características de segunda população em aglomerados globulares afetam visualmente o espectro sintético estelar. Nesse capítulo, exploramos os mesmos efeitos no espectro integrado de aglomerados globulares.

Na Seção 5.1 apresentamos o processo usado na síntese de espectros integrados de AGs, tratando da soma ponderada do fluxo de cada estrela membro. Na Seção 5.2 confrontamos nossos espectros sintéticos integrados com os espectros integrados de AGs observados, comparando modelos e observações em termos de resíduo, ADEV e índices espectrais de Lick.

\subsection{Síntese de espectros integrados}

Para construir o espectro sintético integrado dos AGs deste trabalho, empregamos o método utilizado por Martins et al. (2019). Daquele trabalho, temos o CMD de cada aglomerado convertido para o espaço de parâmetros $T_{\text {eff }}$ vs. $\log g$. Com isto, os autores construíram espectros integrados através da soma dos espectros das estrelas individuais (obtidos a partir de uma biblioteca de espectros), com cada espectro estelar ponderado por um fator $C_{i}$, conforme:

$$
F_{\lambda}=\sum_{i=1}^{N} f_{\lambda, i} C_{i}
$$

onde $f_{\lambda, i}$ é o fluxo no elemento em comprimento de onda $\lambda$ do espectro sintético da $i$-ésima 
estrela do AG. $F_{\lambda}$, portanto, é o fluxo somado em cada elemento de comprimento de onda $\lambda$ das $N$ estrelas do AG. O fator $C_{i}$ é o peso da contribuição do fluxo de cada estrela, dado em função da magnitude visual absoluta $M_{V, i}$ e normalizada pelo fluxo integradq ${ }^{1}$ em comprimento de onda do espectro estelar $f_{\lambda, i}$, conforme:

$$
C_{i}=\frac{10^{\frac{-M_{V, i}}{2.5}}}{\int f_{\lambda, i} d \lambda} .
$$

onde $f_{\lambda, i}$ está normalizado de modo que $\int f_{\lambda, i} d \lambda_{i}=1$.

Assim, para cada um dos aglomerados globulares da nossa grade (vide Tabela 4.1, do capítulo anterior) aplicamos a integração dos respectivos espectros sintéticos usando as Equações 5.1 e 5.2 . Com isso, obtemos dois espectros integrados sintéticos para cada aglomerado: um adotando a mistura " $\alpha+$ ", relativa à primeira geração de estrelas, e o outro a mistura " $\alpha+, \mathrm{He}+, \Delta \mathrm{CNONa",} \mathrm{relativa} \mathrm{à} \mathrm{segunda} \mathrm{geração.}$

Na Figura 5.1, ilustramos os espectros sintéticos integrados que representam os dois padrões de abundâncias químicas calculados acima (i.e., primeira e segunda gerações de estrelas) para NGC 1904 (painel superior), NGC 0104 (painel do meio) e NGC 5927 (painel inferior) - ou seja, do AG menos ao mais metálico (Tabela 4.2). No gráfico inferior de todos os paineis mostramos o resíduo entre os espectros sintéticos do gráfico superior. Em todos os paineis de 5.1, os espectros foram calculados com resolução amostral $R_{\lambda}=300000 \mathrm{e}$ espectral $R_{\mathrm{LSF}}=10000$ e degradados arbitrariamente para melhor visualizaçãd no intervalo em comprimento de onda de 3727 de $9300 \AA$ no qual produzimos a nova lista de opacidades atômicas calibrada com o Sol (Subseção 3.4.4).

As Figuras 5.2 e 5.3 apresentam as mesmas informações da Figura 5.1, mas comparando os espectros sintéticos com os espectros observados por Schiavon e WAGGS, respectivamente. Os espectros sintéticos foram interpolados para os elementos em comprimento de onda e convoluídos para a resolução espectral de cada observação (Seção 2.3). Em 5.2 incluímos os espectros sintéticos calculados em Martins et al. (2019) com os modelos de Coelho (2014) na resolução espectral das observações por Schiavon et al. (2005).

\footnotetext{
${ }^{1}$ Importante: $F_{\lambda} \neq \int f_{\lambda, i} d \lambda$.

${ }^{2}$ Em todas as convoluções utilizamos o mesmo fator gaussiano $\sigma=200$ no módulo gaussian_filter, do pacote SCIPY.
} 

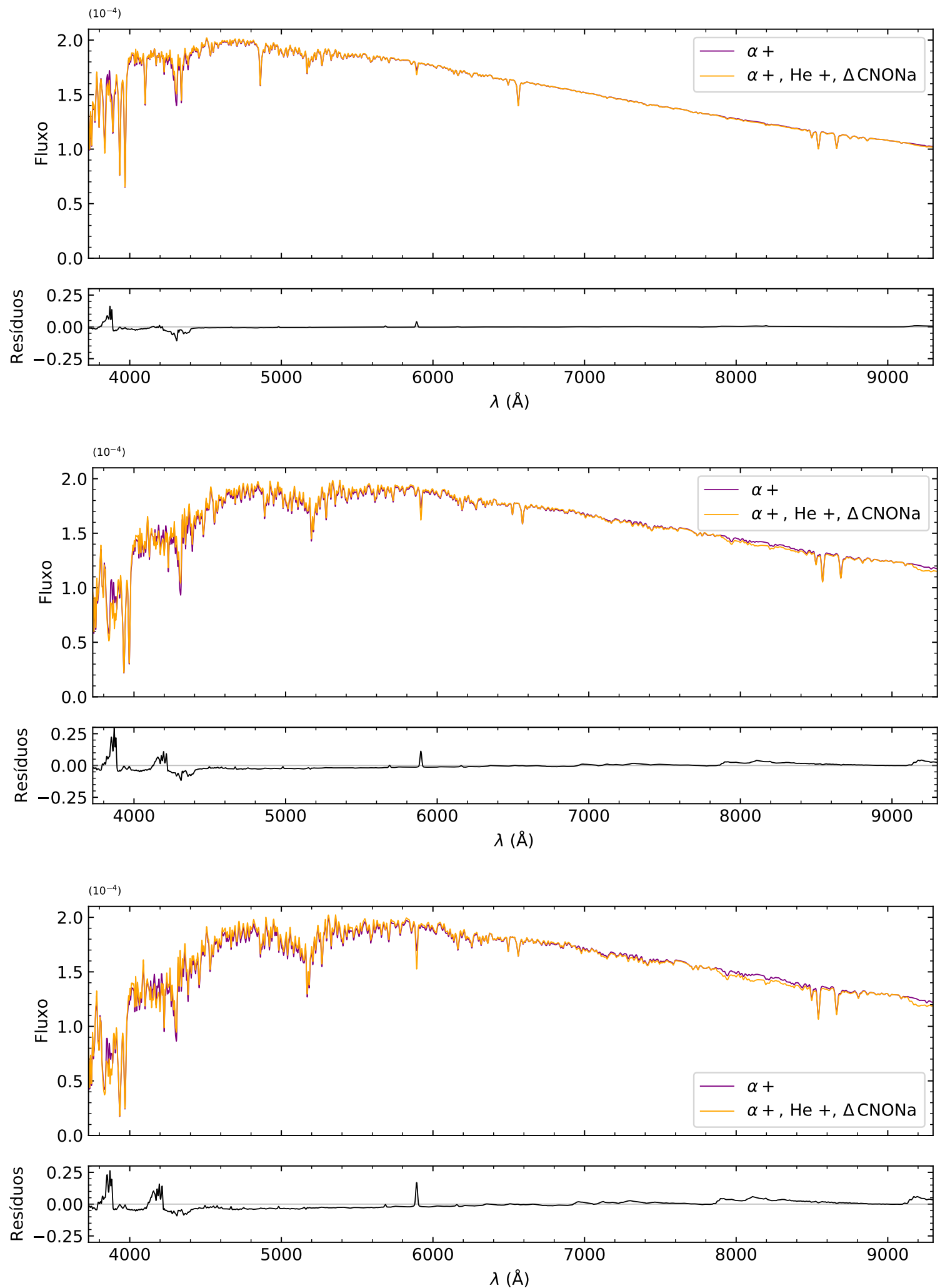

Figura 5.1: Gráfico superior de cada painel: espectros sintéticos da primeira (roxo) e segunda (laranja) gerações de estrelas de NGC 1904 (painel superior), NGC 0104 (painel do meio) e NGC 5927 (painel inferior), de 3727 a $9300 \AA$ A Gráfico inferior de cada painel: resíduos entre os espectros sintéticos no mesmo intervalo do respectivo gráfico superior. 

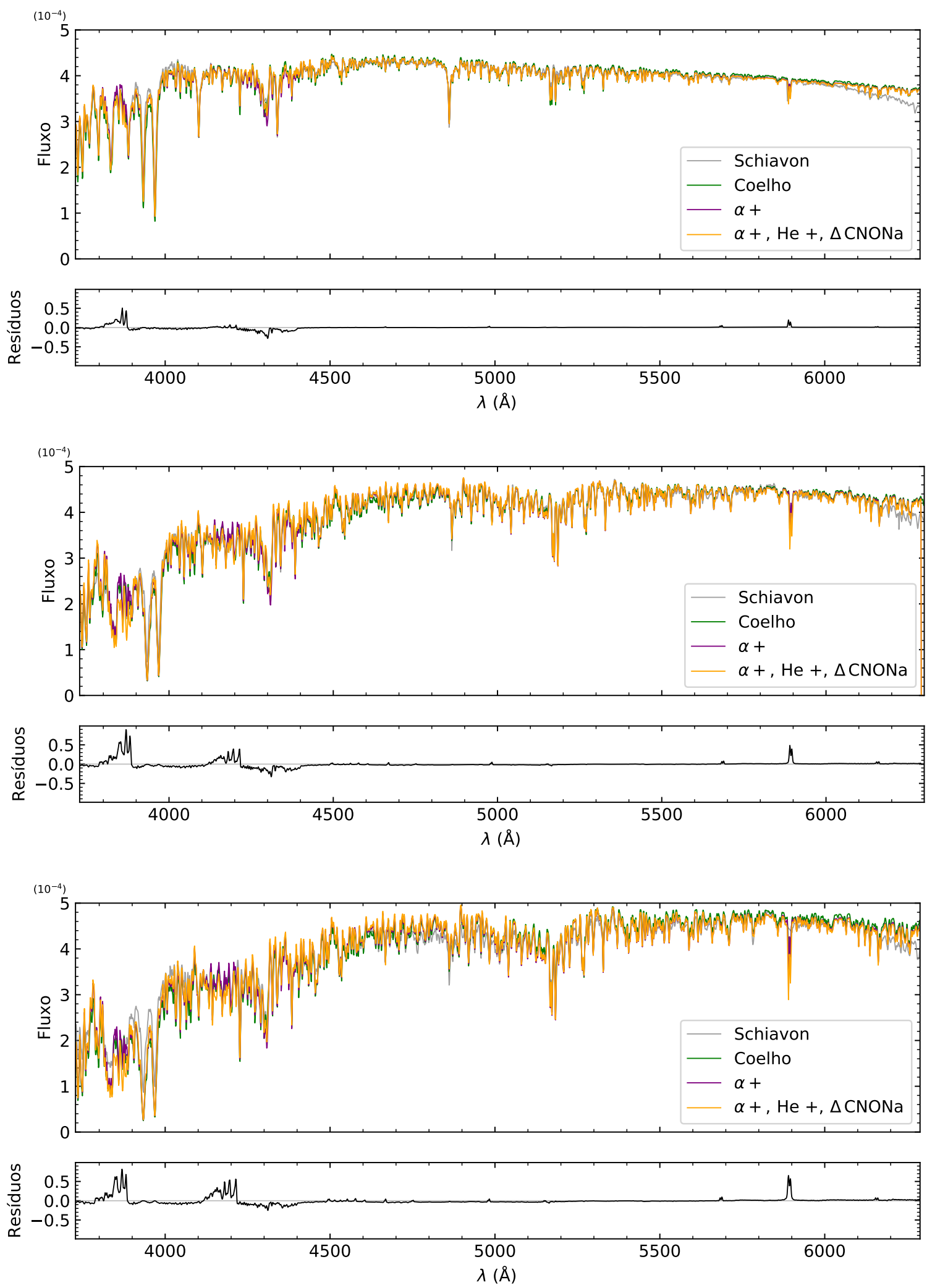

Figura 5.2: Gráfico superior de cada painel: espectros sintéticos da primeira (roxo) e segunda (laranja) gerações de estrelas de NGC 1904 (painel superior), NGC 0104 (painel do meio) e NGC 5927 (painel inferior) comparados com os espectros observados por Schiavon (cinza) e sintéticos calculados com a biblioteca de Coelho (2014) (verde) em Martins et al. (2019), de 3600 a 6300 Å. Gráfico inferior de cada painel: resíduos entre os espectros sintéticos no mesmo intervalo do respectivo gráfico superior. 

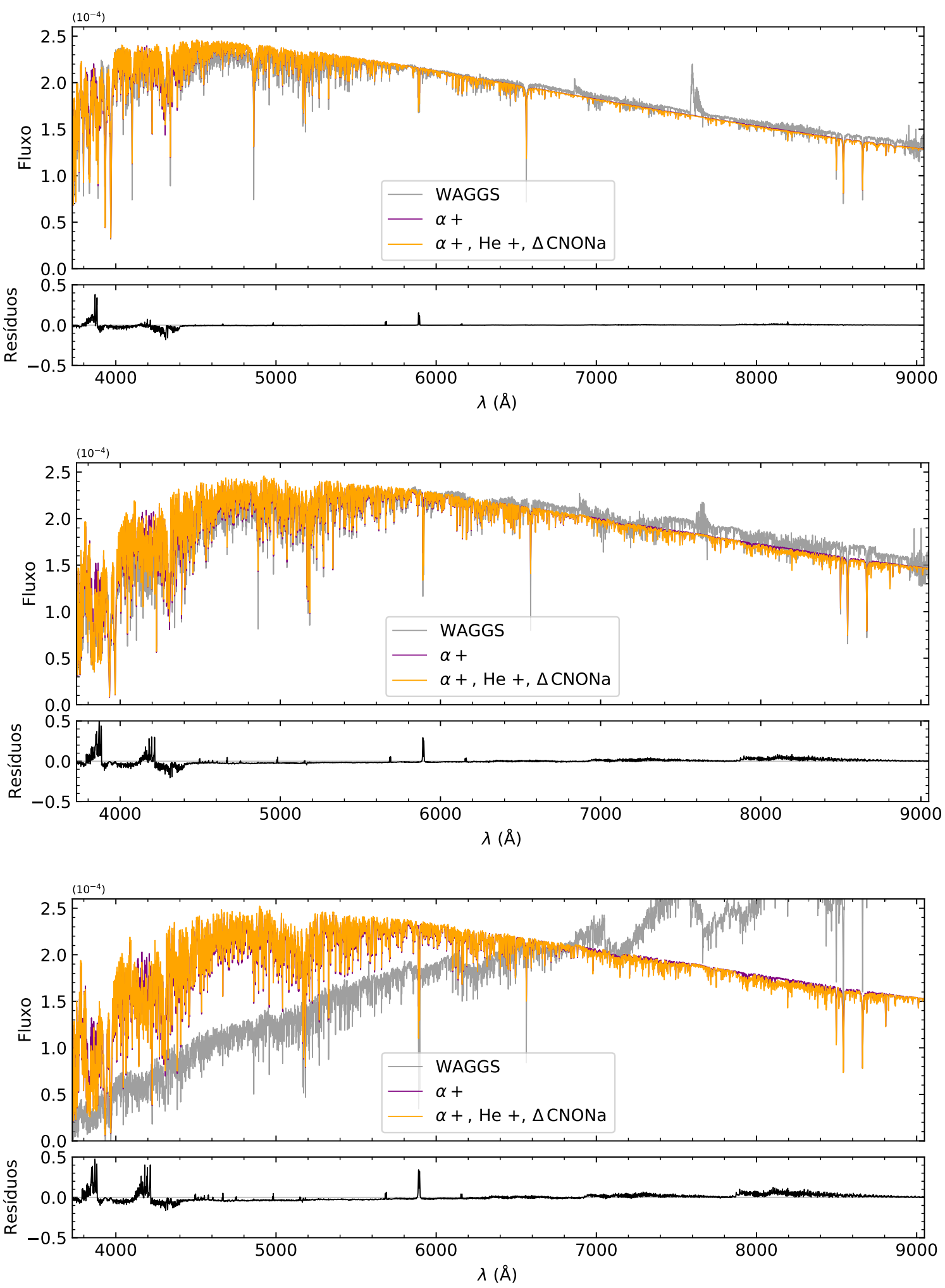

Figura 5.3: Gráfico superior de cada painel: espectros sintéticos da primeira (roxo) e segunda (laranja) gerações de estrelas de NGC 1904 (painel superior), NGC 0104 (painel do meio) e NGC 5927 (painel inferior) comparados com os espectros observados por WAGGS (cinza). Gráfico inferior de cada painel: resíduos entre os espectros sintéticos no mesmo intervalo do respectivo gráfico superior. 
Em todos os paineis das Figuras 5.1, 5.2 e 5.3, a partir dos resíduos, verificamos que a banda mais afetada é a de CN, tal como vimos na Figura 4.2. Além disso, o impacto na banda CN parece depender da metalicidade. Como mencionamos, nestas três figuras, do painel superior ao inferior, os gráficos estão organizados do AG menos ao mais metálico. Assim, observamos que, próximo a $\sim 5890 \AA$, a região característica ao $\mathrm{Na} D$ é afetada pela variação em $\mathrm{Na}$ e parece aumentar com a metalicidade. E, assim como na Figura 4.3, em relação ao $\mathrm{O}$, não conseguimos diferenciar os espectros sintéticos qualitativamente. Por fim, notamos que o aumento em resolução espectral também afeta a qualidade com que as variações adotadas impactam os espectros, sendo o resíduo mais ruidoso quanto maior a resolução estudada.

É importante ressaltar que, embora tenhamos diferenciado as gerações de estrelas com duas misturas químicas, assim como em Coelho et al. (2011), configurando uma comparação entre duas SSPs, o espectro integrado calculado com base em Coelho (2014) é relativo à uma única mistura. Por este motivo, as nossas comparações quantitativas são feitas separadamente: comparando a performance ADEV de cada espectro sintético com a observações usando Coelho (2014) e os índices de lick dos espectros sintéticos deste trabalho com os reportados em Coelho et al. (2011).

\subsection{Comparação com as observações}

Assim como avaliamos a performance dos nossos espectros sintéticos calculados com a nova lista de opacidades atômicas em relação ao Sol e Arcturus, julgamos oportuno aplicar o mesmo procedimento com os nossos espectros integrados sintéticos em relação aos espectros observados dos AGs.

Para essa comparação, consideramos cinco intervalos em comprimento de onda. O intervalo mais abrangente se inicia no mesmo valor em comprimento de onda que inicia a nova lista de opacidades atômicas (i.e., $\lambda=3276,702 \AA$ ) e termina no mesmo valor em comprimento de onda que termina os espectros observados de referência. Os outros quatro intervalos são regiões características dos índices de Lick identificados em Coelho et al. (2011) como sendo mais sensíveis a múltiplas populações. A Tabela 5.1 mostra as regiões consideradas para o cálculo do ADEV. 
Tabela 5.1 - Regiões consideradas no cálculo da performance dos espectros integrados sintéticos. Schiavon e WAGGS se referem à intervalos que terminam no mesmo valor em comprimento de onda das observações de Schiavon et al. (2005) e Usher et al. (2017), respectivamente. Os últimos quatro intervalos referem-se às bandas características do Índice de lick.

\begin{tabular}{|c|c|c|}
\hline ID & $\lambda_{\text {inicial }}(\AA)$ & $\lambda_{\text {final }}(\AA)$ \\
\hline Schiavon & 3726,702 & 6300,000 \\
WAGGS & 3726,702 & 9300,000 \\
$\mathrm{CN}_{1,2}$ & 4080,125 & 4284,125 \\
$\mathrm{Ca}_{42227}$ & 4211,000 & 4251,000 \\
$\mathrm{G} 4300$ & 4266,375 & 4335,125 \\
$\mathrm{Na} D$ & 5860,625 & 5948,125 \\
\hline
\end{tabular}

Vimos que os espectros de AGs como observados por Schiavon et al. (2005) e WAGGS (Usher et al., 2017) são diferentes em termos de estratégia observacional, cobertura em comprimento de onda e resolução espectral $R_{\mathrm{LSF}}$ (Seção 2.3). Em Schiavon et al. (2005) os espectros cobrem de 3600 a $6300 \AA$ e a $R_{\mathrm{LSF}}$ varia, respectivamente, de $\sim 1160$ a 2030 . Em (Usher et al., 2017), os espectros cobrem de 3270 a $9055 \AA$, com a $R_{\mathrm{LSF}}$ variando respectivamente, de $\sim 3837$ a 7763. Assim, convoluímos nossos espectros sintéticos de $R_{\mathrm{LSF}}=10000$ para a resolução das observações baseado em scripts desenvolvidos por Me. G. Gonçalves e Prof. Dra. Paula R. T. Coelho (comunicação privada). A dependência da resolução com comprimento de onda para os espectros WAGGS foi obtida de Gonçalves et al. (2020).

Como relatado na Seção 2.3, os espectros observados do aglomerado NGC 5927, como obtidos por Schiavon e WAGGS, diferem significativamente. C. Usher (comunicação privada) nos forneceu espectros WAGGS extraídos com e sem a estrela LPV, ilustrados na Figura 5.4. A "quebra" nos espectros ocorre porque os espectros ilustrados ainda não são calibrados em fluxo ou escalonados entre as grades B7000 e R7000. Como não foi possível obter os espectros WAGGS calibrados em fluxo de NGC 5927 sem a estrela LPV, em tempo para finalização deste trabalho, utilizamos somente os espectros de Schiavon nesta análise de performance dos espectros integrados sintéticos. 


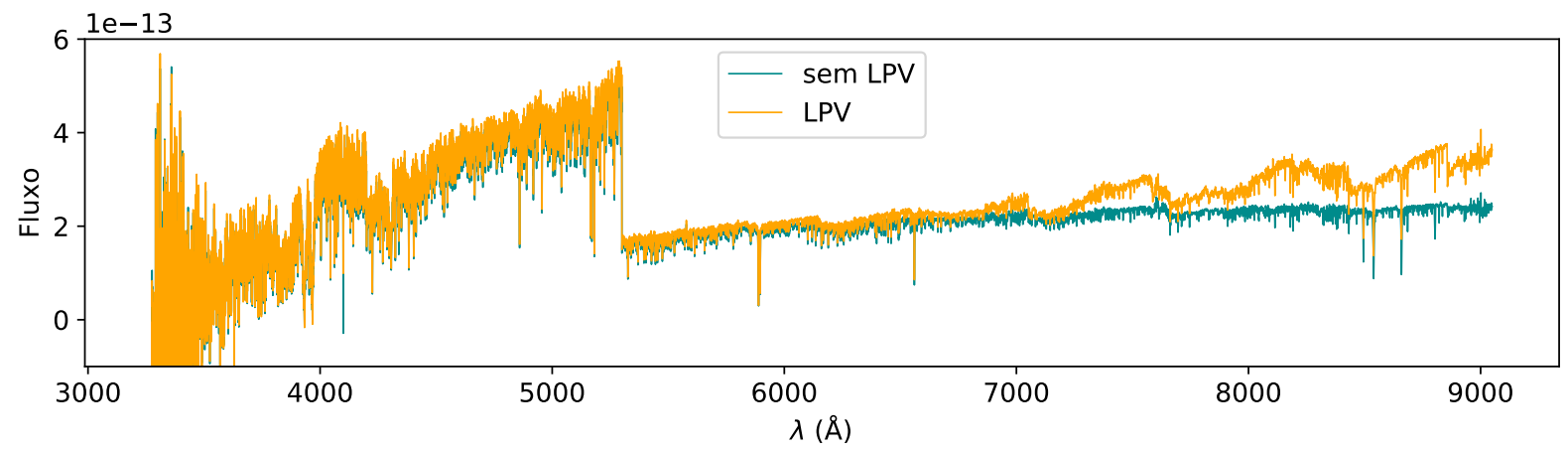

Figura 5.4: Espectros observados de NGC 5927 por WAGGS sem a estrela LPV (ciano) e com a LPV (laranja). Veja Seção 2.3 para mais detalhes.

Para cada AG do nosso estudo, a Tabela 5.2 compila a performance dos espectros integrados sintéticos em comparação aos espectros observados de Schiavon ${ }^{3}$. São apresentados os ADEV calculados para os espectros integrados de Martins et al. (2019) (calculados a partir dos espectros estelares de Coelho 2014) e dos nossos espectros calculados com as duas hipóteses de mistura química, i.e., " $\alpha+$ " ("Ger ${ }_{1}$ ") e " $\alpha+, \mathrm{He}+, \Delta \mathrm{CNONa"} \mathrm{("Ger} 2$ "), nas regiões estabelecidas na Tabela 5.1. A mistura química dos espectros de Coelho (2014) é semelhante à nossa $\mathrm{Ger}_{1}$. Colorimos as células da Tabela 5.2 cujo valor ADEV foi o menor dentre as três comparações em uma mesma região. Verificamos que a maioria dos nossos espectros sintéticos representa melhor os espectros observados, indicando uma melhora quanto aos ingredintes usados na síntese espectral em relação àqueles usados em Coelho (2014). Especificamente para NGC 1904 e NGC 0104, parece haver uma preferência pela hipótese química da Ger $_{2}$. No caso de NGC 5927a preferência indicada é pela Ger . $_{\text {. }}$

De modo geral, a avaliação da performance dos espectros sintéticos está relacionada à qual SSP melhor representa o espectro observado do AG. Contudo, ressaltamos que, teoricamente, o espectro observado abriga as duas hipóteses de misturas, $\mathrm{Ger}_{1}$ e $\mathrm{Ger}_{2}$, e que por ora não tentamos modelar os espectros integrados observados com o quanto de cada geração - mistura química - é necessário para representá-los. Para melhor avaliar o impacto nas linhas características das MPs em AGs no espectro integrado sintético entre as duas gerações, recorremos aos Índices de Lick, discutido na seção a seguir.

\footnotetext{
${ }^{3}$ Veja os resultados obtidos em relação ao espectro observado por WAGGS na Tabela C.1 no Apêndice.
} 
Tabela 5.2 - Comparação entre os ADEVs calculados com os espectros convoluídos em relação ao espectro observado por Schiavon e o espectro calculado com os modelos de Coelho (2014), nas regiões do Índice de Lick e intervalo lambda com início correspondente ao estudo da nova lista atômica e com fim ao espectro observado por Schiavon.

\begin{tabular}{|c|c|c|c|c|c|}
\hline NGC & ID & Região $(\AA)$ & Coelho & Ger $_{1}$ & Ger $_{2}$ \\
\hline 1904 & Schiavon & 3726,702 a 6300,000 & 0,02670 & 0,02067 & 0,01968 \\
1904 & $\mathrm{CN}_{1,2}$ & 4080,125 a 4284,125 & 0,01677 & 0,01263 & 0,01261 \\
1904 & Ca4227 & 4211,000 a 4251,000 & 0,02317 & 0,01544 & 0,01444 \\
1904 & G4300 & 4266,375 a 4335,125 & 0,02906 & 0,03909 & 0,02457 \\
1904 & NaD & 5860,625 a 5948,125 & 0,01333 & 0,01443 & 0,01356 \\
\hline 0104 & Schiavon & 3726,702 a 6300,000 & 0,03566 & 0,03857 & 0,03533 \\
0104 & CN 1,2 & 4080,125 a 4284,125 & 0,03598 & 0,03824 & 0,02494 \\
0104 & Ca4227 & 4211,000 a 4251,000 & 0,04045 & 0,04193 & 0,03327 \\
0104 & G4300 & 4266,375 a 4335,125 & 0,03644 & 0,0597 & 0,04267 \\
0104 & NaD & 5860,625 a 5948,125 & 0,01034 & 0,01214 & 0,01442 \\
\hline 5927 & Schiavon & 3726,702 a 6300,000 & 0,06113 & 0,05172 & 0,05495 \\
5927 & CN 1,2 & 4080,125 a 4284,125 & 0,04724 & 0,04936 & 0,03738 \\
5927 & Ca4227 & 4211,000 a 4251,000 & 0,06177 & 0,06465 & 0,05591 \\
5927 & G4300 & 4266,375 a 4335,125 & 0,05036 & 0,06522 & 0,05195 \\
5927 & NaD & 5860,625 a 5948,125 & 0,02937 & 0,02735 & 0,04918 \\
\hline
\end{tabular}




\subsection{Comparação com a literatura}

O sistema de índices Lick/IDS4 (Worthey et al., 1994; Worthey e Ottaviani, 1997; Vazdekis e Arimoto, 1999) foi elaborado para identificar regiões em espectros estelares que fossem sensíveis à temperatura e à metalicidade. Seu uso foi estendido ao estudo de populações estelares, desde aglomerados globulares à galáxias, onde tem sido usado na deteminação de idades e metalicidades desses sistemas (e.g. Proctor et al., 2004; Lilly e Alvensleben, 2006; Coelho et al., 2011; Barbosa et al., 2016, 2018; Coelho et al., 2020, Rodríguez-Merino et al., 2020).

Neste sistema de índices, são definidos três intervalos de comprimento de onda (ou "bandas"): um na região característica do índice que se deseja calcular e, a partir desta região, um intervalo mais ao azul e outro mais ao vermelho do espectro para definir o contínuo. Índices de moléculas são dados em Magnitudes (M) e índices de atómos são dados em Angtroms (A). A Tabela 5.3 relaciona os índices que usamos neste trabalho, as regiões características de cada um e sua unidade de medida (A ou M), bem como as respectivas bandas do pseudocontínuo Azul e Vermelho.

Calculamos os Índices de Lick da Tabela 5.3 nos espectros integrados sintéticos do nosso trabalho, usando o programa LECTOR $\left.\right|^{5}$ disponível no website de A. Vazdekis. Para avaliar o impacto das duas gerações de estrelas, calculamos a difereça entre os índices da primeira e segunda gerações, i.e., " $\alpha+$ " ("Ger ${ }_{1}$ ) e " $\alpha+, \mathrm{He}+, \Delta \mathrm{CNONa}$ ("Ger 2 "), conforme $\Delta \boldsymbol{I}_{\text {Branco }}=\mathrm{Ger}_{1}-\mathrm{Ger}_{2}$, e comparamos com os resultados obtidos por Coelho et al. (2011). Naquele trabalho, contudo, os espectros integrados foram calculados por síntese de isócronas, usando as isócronas de BaSTI (Pietrinferni et al., 2006), e somente para metalicidades típicas de NGC $0104([\mathrm{Fe} / \mathrm{H}]=-0,7)$.

A Tabela 5.4 apresenta os valores obtidos por Coelho et al. (2011) e os calculados neste trabalho, $\Delta \boldsymbol{I}_{\text {Coelho }}$ e $\Delta \boldsymbol{I}_{\text {Branco }}$, respectivamente. Adotamos aqui a mesma resolução espectral que Coelho et al. (2011), de $R_{\mathrm{LSF}}=10000$. As colunas Schiavon e WAGGS

\footnotetext{
4 "Lick" refere-se ao Observatório Lick, dos Observatórios da Universidade da Califórnia (UCO) e "IDS" à "Image Dissector Scanner", scanner dissector de imagem, em tradução livre.

5 http://research.iac.es/galeria/vazdekis//vazdekis_software.html
} 
referem-se aos índices calculados nos respectivos espectros observados, Schiavon et al. (2005) ( $\boldsymbol{I}_{\text {Schiavon }}^{\text {obs }}$ ) e Usher et al. (2017) ( $\boldsymbol{I}_{\text {WAGGS }}^{\text {obs }}$. À princípio, notamos que os valores dos índices são diferentes entre as observações, porém ressaltamos que seus espectros estão em resoluções distintas. Por isto, neste momento, não comparamos diretamente estes valores com os nossos, Ger $_{1}$ e $\mathrm{Ger}_{2}$; consideramos os Índices de Lick relativos às observações somente como uma referência (uma "verificação de sanidade") para avaliar nossos resultados e discutimos mais adiante sobre o efeito da resolução espectral.

Verificamos que os índices que nós calculamos são compatíveis em ordem de grandeza com os das observações. Contudo, a diferença entre $\boldsymbol{I}_{\text {Coelho }}$ e $\boldsymbol{I}_{\text {Branco }}$ pode ser devida aos diferentes ingredientes usados para modelagem de atmosfera estelar e síntese espectral e o espaço de parâmetros estelares para o espectro sintético representando NGC 0104 (isócrona versus CMD observado). Estes fatores podem influenciar o resultado do cálculo dos índices, assim como a resolução espectral.

Tabela 5.3 - Bandas características dos índices e respectivos pseudocontínuos Azul e Vermelho dos índices de Lick/IDS estudados nesse trabalho (ver texto para detalhes). Índices de moléculas são dados em Magnitudes (M) e índices de atómos são dados em Angtroms (A).

\begin{tabular}{|c|c|c|c|c|}
\hline Índice & Contínuo Azul $(\AA)$ & Região $(\AA)$ & Contínuo Vermelho $(\AA)$ & $(\mathrm{A} / \mathrm{M})$ \\
\hline $\mathrm{H} \delta_{F}$ & 4057,250 a 4088,500 & 4091,000 a 4112,250 & 4114,750 a 4137,250 & $\mathrm{~A}$ \\
$\mathrm{CN}_{1}$ & 4080,125 a 4117,625 & 4142,125 a 4177,125 & 4244,125 a 4284,125 & $\mathrm{M}$ \\
$\mathrm{CN}_{2}$ & 4083,875 a 4096,375 & 4142,125 a 4177,125 & 4244,125 a 4284,125 & $\mathrm{M}$ \\
$\mathrm{Ca} 4227$ & 4211,000 a 4219,750 & 4222,250 a 4234,750 & 4241,000 a 4251,000 & $\mathrm{~A}$ \\
$\mathrm{G} 4300$ & 4266,375 a 4282,625 & 4281,375 a 4316,375 & 4318,875 a 4335,125 & $\mathrm{~A}$ \\
$\mathrm{H} \gamma_{F}$ & 4283,500 a 4319,750 & 4331,250 a 4352,250 & 4354,750 a 4384,750 & $\mathrm{~A}$ \\
$\mathrm{H} \beta$ & 4827,875 a 4847,875 & 4847,875 a 4876,625 & 4876,625 a 4891,625 & $\mathrm{~A}$ \\
$\mathrm{Mg}{ }_{b}$ & 5142,625 a 5161,375 & 5160,125 a 5192,625 & 5191,375 a 5206,375 & $\mathrm{~A}$ \\
$\mathrm{Fe} 5270$ & 5233,150 a 5248,150 & 5245,650 a 5285,650 & 5285,650 a 5318,150 & $\mathrm{~A}$ \\
$\mathrm{Fe} 5335$ & 5304,625 a 5315,875 & 5312,125 a 5352,125 & 5353,375 a 5363,375 & $\mathrm{~A}$ \\
$\mathrm{Fe} 5406$ & 5376,250 a 5387,500 & 5387,500 a 5415,000 & 5415,000 a 5425,000 & $\mathrm{~A}$ \\
$\mathrm{Na} D$ & 5860,625 a 5875,625 & 5876,875 a 5909,375 & 5922,125 a 5948,125 & $\mathrm{~A}$ \\
\hline
\end{tabular}


Na Tabela 5.5 apresentamos as variações $\Delta \boldsymbol{I}$ dos índices calculados com espectros em diferentes resoluções espectrais: $\Delta \boldsymbol{I}_{\text {Coelho }}$ e $\Delta \boldsymbol{I}_{\text {Branco }}$ derivam de espectros sintéticos com $R_{\mathrm{LSF}}=10000$, enquanto que $\Delta \boldsymbol{I}_{\text {Schiavon }}^{\text {sint }}$ e $\Delta \boldsymbol{I}_{\text {WAGGS }}^{\text {sint }}$ derivam de espectros sintéticos convoluídos para a resolução dos espectros observado\$ ${ }^{6}$ por Schiavon et al. (2005) e Usher et al. (2017), respectivamente. Verificamos que os resultados são compatíveis em ordem de grandeza e que os valores dos índices dependem da resolução espectral (como já sabido da literatura).

Tabela 5.4 - Variação dos Índices de Lick para NGC 0104, calculados com os espectros observados por Schiavon et al. 2005) ( $\boldsymbol{I}_{\text {Schiavon }}^{\text {obs }}$ ) e Usher et al. 2017) ( $\Delta \boldsymbol{I}_{\text {WAGGS }}^{\text {obs }}$ ) e com os espectros sintéticos para a primeira e segunda gerações ("Ger ${ }_{1}$ e "Ger 2 ", respectivamente), e respectivas variações dos índices calculadas em Coelho et al. (2011) $\left(\Delta \boldsymbol{I}_{\text {Coelho }}\right)$ e as deste trabalho entre as duas gerações $\left(\Delta \boldsymbol{I}_{\text {Branco }}\right)$.

\begin{tabular}{|c|c|c|c|c|c|c|}
\hline Índice & $\boldsymbol{I}_{\text {Schiavon }}^{\text {obs }}$ & $\boldsymbol{I}_{\text {WAGGS }}^{\text {obs }}$ & Ger $_{\mathbf{1}}$ & $\mathbf{G e r}_{\mathbf{2}}$ & $\Delta \boldsymbol{I}_{\text {Coelho }}$ & $\Delta \boldsymbol{I}_{\text {Branco }}$ \\
\hline $\mathrm{CN}_{1}$ & 0,044 & 0,065 & 0,071 & 0,128 & 0,084 & 0,057 \\
$\mathrm{CN}_{2}$ & 0,082 & 0,106 & 0,077 & 0,136 & 0,087 & 0,059 \\
$\mathrm{Ca} 4227$ & 0,876 & 0,851 & 2,309 & 1,866 & $-0,651$ & $-0,443$ \\
$\mathrm{G} 4300$ & 4,867 & 5,242 & 6,894 & 5,936 & $-0,572$ & $-0,958$ \\
$\mathrm{Na} D$ & 1,748 & 2,266 & 1,045 & 1,953 & 1,346 & 0,908 \\
\hline
\end{tabular}

Tabela 5.5 - Variações dos Índices de Lick para NGC 0104 calculadas com diferentes resoluções espectrais, sendo $\Delta \boldsymbol{I}_{\text {Coelho de }}$ Coelho et al. (2011) e $\Delta \boldsymbol{I}_{\text {Branco }}$ deste trabalho, calculados com resolução $R_{\mathrm{LSF}}=10000$, $\Delta \boldsymbol{I}_{\text {Schiavon }}^{\text {sint }}$ na resolução de Schiavon et al. (2005) e $\Delta \boldsymbol{I}_{\text {WAGGS }}^{\text {sint }}$ na resolução de Usher et al. $(2017)$.

\begin{tabular}{c|c|c|c|c|}
\hline Índice & $\Delta \boldsymbol{I}_{\text {Coelho }}$ & $\Delta \boldsymbol{I}_{\text {Branco }}$ & $\Delta \boldsymbol{I}_{\text {Schiavon }}^{\text {sint }}$ & $\Delta \boldsymbol{I}_{\text {WAGGS }}^{\text {sint }}$ \\
\hline $\mathrm{CN}_{1}$ & 0,084 & 0,057 & 0,065 & 0,064 \\
$\mathrm{CN}_{2}$ & 0,087 & 0,059 & 0,067 & 0,066 \\
$\mathrm{Ca}_{2227}$ & $-0,651$ & $-0,443$ & $-0,440$ & $-0,432$ \\
$\mathrm{G} 4300$ & $-0,572$ & $-0,958$ & $-0,861$ & $-0,827$ \\
$\mathrm{Na} D$ & 1,346 & 0,908 & 0,929 & 0,929 \\
\hline
\end{tabular}

\footnotetext{
${ }^{6}$ Convoluções descritas na Seção 5.2
} 
Na Figura 5.5 mostramos diagramas índice-índice $\left(\mathrm{CN}_{1}, \mathrm{CN}_{2}, \mathrm{Ca} 4227, \mathrm{G} 4300\right.$ e $\mathrm{NaD}$ vs. Fe 5406) referentes aos espectros observados e às duas hipóteses de mistura química medidos nos espectros integrados sintéticos deste trabalho (convoluídos para as resoluções dos espectros observados). Em cada diagrama, para facilitar a visualização, ajustamos funções quadráticas que ilustram linhas de tendência dos índices relativos aos espectros sintéticos. Comparando nossos índices sintéticos com os medidos em observações, notamos diferenças sistemáticas que variam dependendo do AG. Atribuímos essas diferencas a duas possiblidades: 1) problemas com a seleção ou atribuição das opacidades moleculares e/ou atômicas; ou 2) a modelagem da abundância química dos AGs está incorreta. Diferenças entre índices sintéticos e observados tem sido relatado na literatura, seja para estrelas (e.g. Martins e Coelho, 2007) ou para SSPs (e.g. Coelho et al., 2020).

Em todos os diagramas da Figura 5.5, exceto em relação ao G4300, observamos que o valor do índice medido, seja sintético ou observado, aumenta com o índice de Fe5406 que, entre outros, é usado para inferir a abundância de ferro e a metalicidade Z do sistema. Este comportamento dos índices sensíveis a elementos químicos aumentarem com a metalicidade é esperado e já foi relatado na literatura, e.g., Thomas et al. (2003), Lee e Worthey (2005) e Thomas et al. (2011).

Com relação ao efeito das diferentes gerações de MPs, notamos que todos os índices são sensíveis às variações químicas, confirmando os resultados de Coelho et al. (2011). Também notamos, pela primeira vez, que enquanto a diferença para G4300 parece constante, para os índices $\mathrm{CN}_{1}, \mathrm{CN}_{2}, \mathrm{Ca} 4227$ e $\mathrm{Na} D$ elas aumentam com a metalicidade da população estelar.

Na Figura 5.6 repetimos as análise de índices de Coelho et al. (2011), apresentando diagramas similares à Fig. 5.5 mas relacionando o $[\mathrm{MgFe}]^{7}$ e Fe 5406 (índices sensíveis à metalicidade $\mathrm{Z}$ de cada $\mathrm{AG}$ ), com os índices de Balmer $\mathrm{H} \beta, \mathrm{H} \delta_{F}$ e $\mathrm{H} \gamma_{F}$, sensíveis à idade e à variação na fração de massa Y. Recordarmos aqui que, além das variações CNONa, as nossas duas hipóteses de mistura química diferem da fração de massa em He, i.e., com Y $=0,256$ representando a primeira geração de estrelas do aglomerado globular (i.e. " $\alpha+$ ") e $\mathrm{Y}=0,300$ representando a segunda geração (i.e., " $\alpha+$, He+, $\Delta \mathrm{CNONa").} \mathrm{Com} \mathrm{esses}$

\footnotetext{
$7[\mathrm{MgFe}]=\sqrt{\mathrm{Mg}_{\mathrm{b}} \cdot<\mathrm{Fe}>}$, onde $<\mathrm{Fe}>=(\mathrm{Fe} 5270+\mathrm{Fe} 5335) / 2$
} 
diagramas confirmamos o efeito não-negligível das gerações de MPs nos índices de Balmer (exceto $H \beta$ ) para NGC 0104, como relatado em Coelho et al. (2011). Pela primeira vez, verificamos que o mesmo efeitos ocorre para AGs em outros regimes de metalicidade.

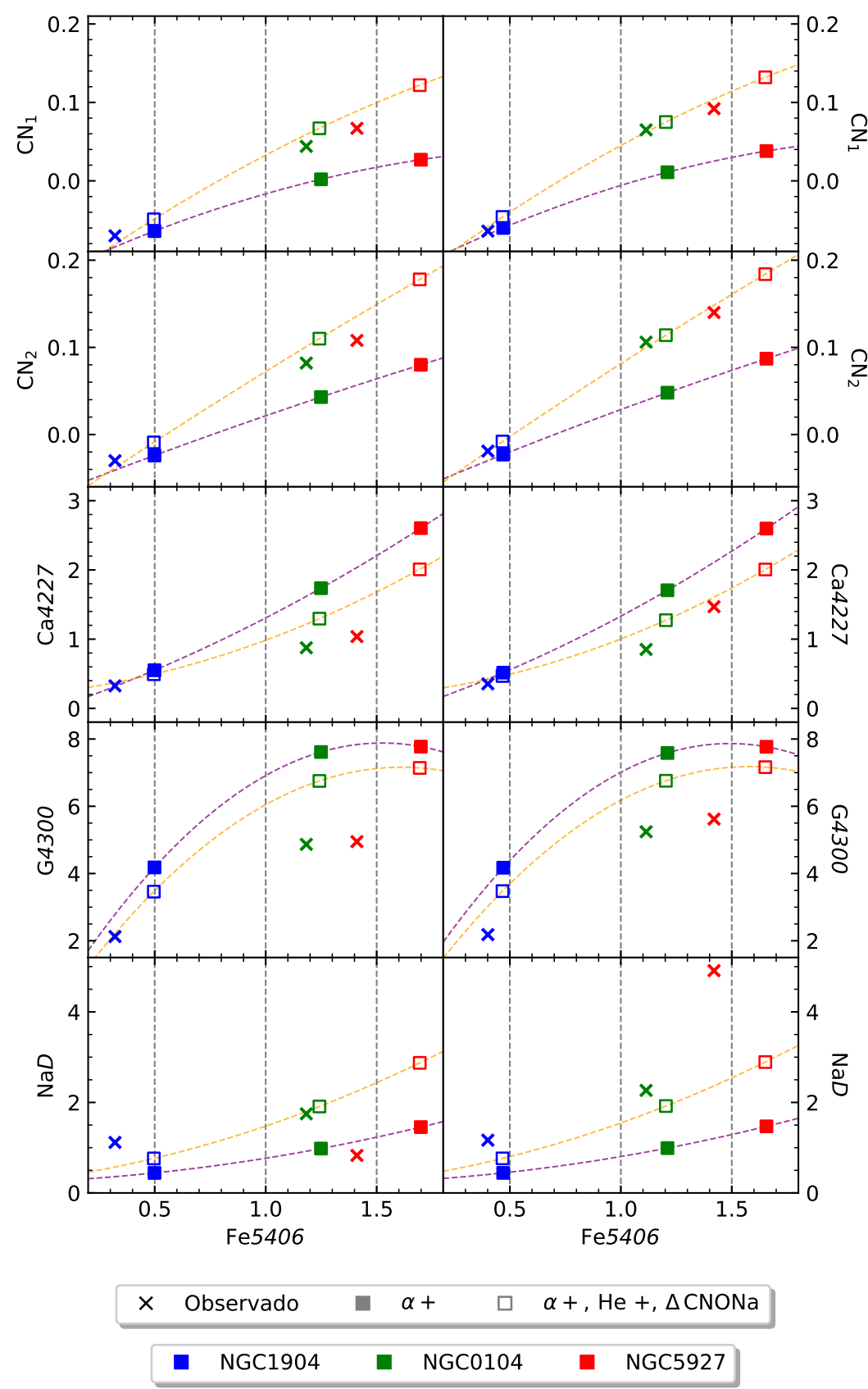

Figura 5.5: Diagramas índice-índice de $\mathrm{CN}_{1}, \mathrm{CN}_{2}$, Ca4227, G4300 e $\mathrm{Na} D$ em função de Fe 5406 (do painel superior ao inferior) das misturas químicas " $\alpha+$ " (quadrado preenchido) e " $\alpha+, \mathrm{He}+, \Delta \mathrm{CNONa"} \mathrm{(qua-}$ drado vazio) para NGC 1904, NGC 0104 e NGC 5927 (quadrados azul, verde e vermelho, respectivamente). Os Índices de Lick com marcador em "x" se referem aos índices calculados com os espectros observados por Schiavon (coluna à esquerda) e WAGGS (coluna à direita). As linhas tracejadas indicam a tendência do índice em relação à primeira (roxo) e segunda (laranja) geracão. 

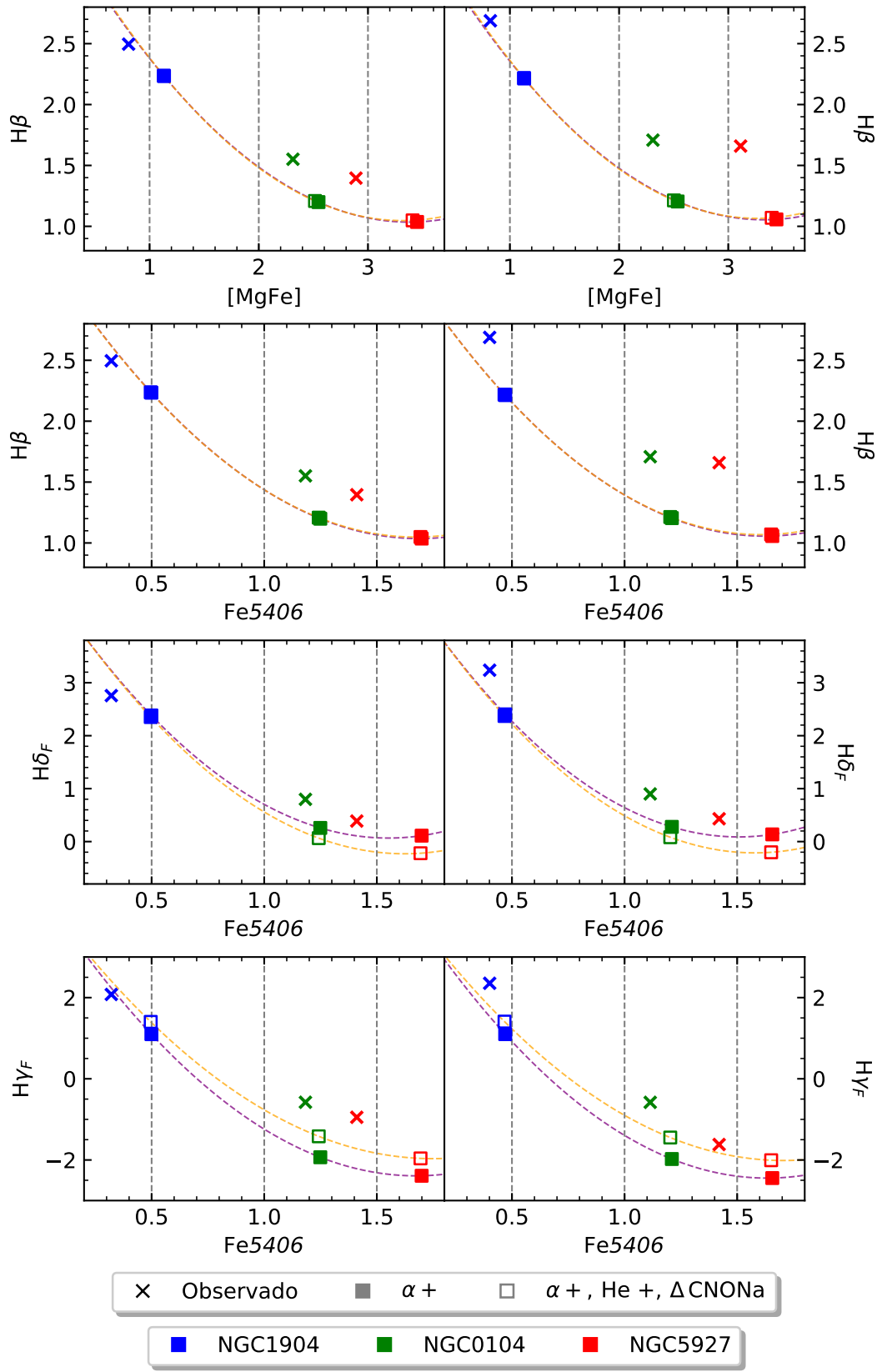

Figura 5.6: Diagramas índice-índice de $\mathrm{H} \beta, \mathrm{H} \delta_{F}$ e $\mathrm{H} \gamma_{F}$ em função de [MgFe] (paineis superiores) e Fe 5406 (demais paineis) das misturas químicas " $\alpha+$ " (quadrado preenchido) e " $\alpha+, \mathrm{He}+, \Delta \mathrm{CNONa"} \mathrm{(quadrado}$ vazio) para NGC 1904, NGC 0104 e NGC 5927 (quadrados azul, verde e vermelho, respectivamente). Os Índices de Lick com marcador em " $x$ " se referem aos índices calculados com os espectros observados por Schiavon (coluna à esquerda) e WAGGS (coluna à direita). As linhas tracejadas indicam a tendência do índice em relação à primeira (roxo) e segunda (laranja) geracão. 


\section{Capítulo 6}

\section{Conclusões}

Foi uma aventura e tanto. Este projeto de mestrado teve como principal objetivo expandir os resultados obtidos por Coelho et al. (2011) para outras metalicidades. Para isso, muito esforço foi dedicado a estruturar e aprender os fundamentos que permeiam todo o escopo do projeto: a modelagem de atmosferas e espectros estelares e as opacidades que são usadas como ingredientes. Isto nos levou a executar projetos paralelos (como o desenvolvimento de um programa) que tiveram influência direta nos resultados deste trabalho e, com isso, avaliamos que contruímos mais e fomos além daquilo que havíamos previsto no início do projeto.

Nosso trabalho realizado com os programas de síntese espectral e modelagem de atmosfera SYNTHE e ATLAS12 culminou na criação de um processo computacional com a capacidade de calcular modelos de atmosfera e seus espectros sintéticos em série e de forma bastante eficiente, o PyGLobsters. Com este programa, por exemplo, conseguimos calcular todos os espectros da grade $\Theta^{1}$ em apenas dois dias, o que viabilizou análises e correções mais ágeis.

Foi crucial para este projeto a escolha das abundâncias químicas solares, opacidades atômicas e moleculares que melhor pudessem, juntos, representar os espectros observados do Sol e Arcturus. Apesar do caráter degenerado entre estes ingredientes da síntese espectral, selecionamos a abundância solar Asplund et al. (2009) ao invés de Grevesse e Sauval (1998) e Asplund et al. (2005), por ser a mais atual. Quanto às opacidades moleculares, em particular as espécies $\mathrm{C}_{2}[\mathrm{D}-\mathrm{A}], \mathrm{SiH}, \mathrm{OH}, \mathrm{CN}[\mathrm{A}-\mathrm{X}], \mathrm{CN}[\mathrm{B}-\mathrm{X}]$ e $\mathrm{CH}$, selecionamos a lista

\footnotetext{
${ }^{1}$ Foram 376 espectros em alta resolução amostral $R_{\lambda}=300$ 000, de 300 a $1000 \AA$.
} 
de opacidades cuja autoria fosse a mais atual e/ou demonstrasse, ainda que visualmente, melhor representação do espectro solar. A lista completa das espécies moleculares usadas no trabalho pode ser consultada na Tabela 3.3 .

No caso da seleção das opacidades atômicas usamos uma métrica objetiva, o ADEV, que nos permitiu avaliar a performance de três listas da literatura, em pequenos intervalos de comprimento de onda ao longo de todo o espectro visível. Nesta atividade, as linhas da lista com melhor performance em cada intervalo de comprimento de onda foram selecionadas para compor uma nova lista. No processo, percebemos que há linhas referentes a um mesmo átomo (ou isótopo) em uma lista que têm, principalmente, o valor do comprimento de onda central diferente em outra lista. A esta inconsistência chamamos no trabalho de "linhas migratórias". Estas linhas estavam desaparecidas nos primeiros espectros sintéticos que calculamos, mas após o refinamento na seleção, recuperando as "linhas migratórias", corrigindo o espectro sintético que é calculado com a nova lista de opacidades atômicas.

Assim, após o estudo e seleção dos ingredientes da síntese espectral acima, como apresentamos na Seção 3.4.4, produzimos duas novas listas de opacidades atômicas que resultam em ADEVs menores (melhores) do que os das listas de referência da literatura. Em resumo, nossos espectros sintéticos representam melhor os espectros observados do Sol e Arcturus do que aqueles calculados com as outras listas da literatura estudadas (veja Tabela 3.5).

De posse das novas listas de opacidades, construímos uma grade de espectros sintéticos para avaliar os impactos das anticorrelações químicas nos espectros integrados de AGs. Calculamos 376 espectros sintéticos estelares, divididos entre duas hipóteses de mistura química e três metalicidades, cada qual representando um AG, i.e., NGC 1904, NGC 0104 e NGC 5927 (Seção 4.3). Dos gráficos de resíduos entre os espectros sintéticos da Figura 4.2, calculados com $R_{\mathrm{LSF}}>150000$, as regiões de CN e Na são as mais afetadas, tanto para o Sol quanto para Arcturus, que são as regiões espectrais mais sensíveis às alterações químicas que fizemos, conforme era esperado da literatura. Na região do O o efeito é menos percebido, o que também é esperado, e mostramos estas regiões ampliadas na Figura 4.3 . Concluímos esta etapa exibindo na Figura 4.4 os espectros sintéticos estelares calculados com o menor e maior valores de $T_{\text {eff }}$ e $\log g$ da cobertura da grade para cada metalicidade. 
Desta última figura, verificamos que, em alta resolução espectral, os efeitos das duas misturas são mais evidentes entre estrelas mais frias e menor gravidade superfical, a diferença aumentando com a metalicidade.

A partir da grade de espectros estelares, adotamos o mesmo procedimento de síntese de populações de Martins et al. (2019) para calcular os espectros integrados sínteticos dos nossos AGs. Estes espectros foram confrontados com os espectros integrados observados por Schiavon et al. (2005) e WAGGS Usher et al. (2017). Da Figura 5.1, o gráfico de resíduos aponta para os mesmos efeitos entre espectros sintéticos de diferentes gerações na região de $\mathrm{CN}$ e Na observado na comparação entre os espectros estelares, em concordância com os resultados de Coelho et al. (2011, 2012) para NGC 0104. O proeminência na diferença da linha em $\mathrm{Na}$, assim como o efeito em $\mathrm{CN}$, parece ser mais perceptível com o aumento da metalicidade.

De modo a quantificar o impacto das diferentes gerações de MPs nos espectros integrados sintéticos do aglomerados, calculamos o ADEV entre os espectros observados dos AGs e os nossos sintéticos. Concluímos desta etapa do trabalho que, na maioria dos casos analisados, nossos espectros integrados sintéticos performam melhor do que os espectros calculados em Martins et al. (2019) com base na biblioteca de Coelho (2014). Notamos também que para NGC 5927 parece haver maior preferência pela mistura química da primeira geração em relação à segunda (Tabela 5.2), enquanto que para NGC 1904 e NGC 0104 a preferência tende para a primeira geração.

Para comparar nosso trabalho com a literatura, calculamos os Índices de Lick de $\mathrm{CN}_{1}$, $\mathrm{CN}_{2}, \mathrm{Ca} 4227, \mathrm{G} 4300, \mathrm{Na} D, \mathrm{Fe} 5270, \mathrm{Fe} 5335, \mathrm{Fe} 5406, \mathrm{Mg}_{\mathrm{b}}, \mathrm{H} \beta, \mathrm{H} \delta_{F}$ e $\mathrm{H} \gamma_{F}$ e $[\mathrm{MgFe}] \operatorname{dos}$ espectros integrados sintéticos para NGC 1904, NGC 0104 e NGC 5927. Nossos resultados estão, em ordem de grandeza, de acordo com os calculados por Coelho et al. (2011) (Tabela 5.4). Além disso, confirmando o previsto pela literatura, as diferentes resoluções espectrais afetam os índices calculados, como mostramos na Tabela 5.5. Entendemos que as diferenças entre opacidades adotadas na síntese estelar e os diferentes métodos de síntese de populações explicam as diferenças entre os nossos resultados e os de Coelho et al. (2011). 
Como mostramos nas Figuras 5.5 e 5.6, nossos resultados corroboram o efeito nãonegligível das múltiplas populações nos índices de Balmer (exceto $H \beta$ ) em NGC 0104, conforme apresentado em Coelho et al. (2011). Contudo, pela primeira vez mostramos esses efeitos também para outras metalicidades, representadas por NGC 1904 e NGC 5927. Notamos que enquanto a diferença no índice G4300 é aproximadamente constante, elas aumentam com a metalicidade da população estelar para $\mathrm{CN}_{1}, \mathrm{CN}_{2}$, Ca4227 e $\mathrm{NaD}$.

Por fim, ressaltamos que, à semelhanca de Coelho et al. (2011), as duas gerações de MPs calculadas no trabalho correspondem a SSPs com padrões químicos diferentes, de modo que as estrelas de uma dada SSP são quimicamente homogêneas. No entanto, como vimos, nos AGs reais isso não acontece e o espectro integrado do AG que observamos é uma mistura de populações estelares. As diferenças entre os espectros integrados sintéticos calculados nesse trabalho servem, portanto, como limites superiores do que devemos observar na natureza. 


\section{Perspectivas}

Desde quando iniciei esta jornada, trabalhando com as múltiplas populações nos aglomerados globulares Galácticos, desenvolvi pessoalmente e profissionalmente muitas habilidades técnicas e analíticas que foram aproveitadas em muitas outras situações da minha carreira acadêmica. Com este projeto de mestrado não foi diferente e muitas atividades que gostaríamos de ter feito não puderam ser exploradas.

A primeira parte deste trabalho consistiu em escolher os ingredientes para síntese espectral estelar. As escolhas de abundância solar de referência, opacidades moleculares e opacidades atômicas foram feitas serialmente, sem aprofundar demais no impacto que essas escolhas teriam no espectro final. Por exemplo, quando optamos por Asplund et al. (2009) ao invés de Grevesse e Sauval (1998), nós fixamos as abundâncias químicas sobre as quais as opacidades moleculares e atômicas seriam calculadas. A degenerecência entre essas variáveis pode ter impactado a lista de opacidades atômicas que produzimos. Explorar de forma objetiva a degenerescência entre essas variáveis e o efeito que suas combinações tem no espectro sintético estelar, pode ajudar a realizar calibrações de opacidades mais robustas, que representem melhor os espectros observados estelares.

Da aplicação de nossa grade a espectros integrados de aglomerados, verificamos que há diferenças sistemáticas entre os índices observados e sintéticos, seja em relação a Schiavon ou WAGGS (Figuras 5.5 e 5.6). Apontamos para alguns caminhos que talvez ajudem a elucidar a questão: uma modelagem mais precisa das misturas químicas das diferentes gerações, i.e., das variações de abundâncias CNONa nos AGs pode vir a diminuir essas diferenças; e a calibração das opacidades pode ser melhor desenvolvida considerando as degenerescências comentadas acima. 
Ainda da Figura 5.5, a regresão polinomial quadrática ajudou a perceber como a diferença entre os índices sintéticos muda com a metalicidade. Naquela situação, usamos apenas três AGs cujas metalicidades são bastante diferentes. De posse de um número maior de AGs com seus índices sintéticos medidos, podemos aumentar a amostra nos diagramas de modo a estatisticamente confirmar as previsões neste trabalho.

Por fim, se AGs Galácticos são representantes locais de aglomerados extragaláticos, uma consequência direta e natural deste projeto é o estudo de múltiplas populações em aglomerados fora da nossa Galáxia. Uma vez caracterizados os espectros integrados de MPs com AGs locais, podemos usar modelos de populações como os produzidos neste trabalho para identificar e medir variações químicas em espectros de AGs extragalácticos, ampliando o panorama de estudo deste fenômeno. 
"A Via Láctea geralmente é representada nos mapas astronômicos como uma zona irregular de brilho que circunda os céus e minhas medidas provaram que sua tonalidade esbranquiçada surge de estrelas acumuladas, muito fracas para serem distinguidas a olho nu. (...) as observações detalhadas nas páginas anteriores deste artigo, autorizam-nos a antecipar a fragmentação da Via Láctea, em todas as suas partes diminutas, como consequência inevitável do poder de aglomeração decorrente daquelas preponderantes atrações que têm se mostrado existir em todos os lugares de seu espaço."

(tradução livre do excerto abaixo)

"The milky way is generally represented in astronomical maps as an irregular zone of brightness encircling the heavens, and my star gages have proved its whitish tinge to arise from accumulated stars, too faint to be distinguished by the eye. (...) the observations detailed in the preceding pages of this paper, authorise us to anticipate the breaking up of the milky way, in all its minute parts, as the unavoidable consequence of the clustering power arising out of those preponderating attractions which have been shewn to be every where existing in its compass." 


\section{Referências Bibliográficas}

Allard F., Hauschildt P. H., Model Atmospheres for M (Sub)Dwarf Stars. I. The Base Model Grid, ApJ, 1995, vol. 445, p. 433

Asplund M., Grevesse N., Sauval A. J., The Solar Chemical Composition. In Cosmic Abundances as Records of Stellar Evolution and Nucleosynthesis, vol. 336 of Astronomical Society of the Pacific Conference Series, 2005, p. 25

Asplund M., Grevesse N., Sauval A. J., Scott P., The Chemical Composition of the Sun, ARA\&A, 2009, vol. 47, p. 481

Barbosa C. E., Arnaboldi M., Coccato L., Gerhard O., Mendes de Oliveira C., Hilker M., Richtler T., Sloshing in its cD halo: MUSE kinematics of the central galaxy NGC 3311 in the Hydra I cluster, A\&A, 2018, vol. 609, p. A78

Barbosa C. E., Arnaboldi M., Coccato L., Hilker M., Mendes de Oliveira C., Richtler T., The Hydra I cluster core. I. Stellar populations in the cD galaxy NGC 3311, A\&A, 2016, vol. 589, p. A139

Barbuy B., A Calibration of MG 2 versus [Fe/H] and [Mg/Fe], ApJ, 1994, vol. 430, p. 218

Barbuy B., Chiappini C., Gerhard O., Chemodynamical History of the Galactic Bulge, ARA\&A, 2018, vol. 56, p. 223

Barbuy B., Trevisan J., de Almeida A., PFANT: Stellar spectral synthesis code, Astrophysics Source Code Library, 2018, p. ascl:1812.003

Baron E., Hauschildt P. H., Nugent P., Branch D., Non-local thermodynamic equilibrium effects in modelling of supernovae near maximum light., MNRAS, 1996, vol. 283, p. 297 
Bastian N., Lardo C., Multiple Stellar Populations in Globular Clusters, ARA\&A, 2018, vol. 56 , p. 83

Bernath P. F., Colin R., Revised molecular constants and term values for the X2 and B2+ states of OH, Journal of Molecular Spectroscopy, 2009, vol. 257, p. 20

Bonnarel F., Fernique P., Bienaymé O., Egret D., Genova F., Louys M., Ochsenbein F., Wenger M., Bartlett J. G., The ALADIN interactive sky atlas. A reference tool for identification of astronomical sources, A\&AS, 2000, vol. 143, p. 33

Branco V., Coelho P. R. T., Atomic/molecular opacities and globular cluster spectra, Boletin de la Asociacion Argentina de Astronomia La Plata Argentina, 2020, vol. 61C, p. 85

Brodie J. P., Strader J., Extragalactic Globular Clusters and Galaxy Formation, ARA\&A, 2006, vol. 44, p. 193

Brooke J. S. A., Bernath P. F., Schmidt T. W., Bacskay G. B., Line strengths and updated molecular constants for the $\mathrm{C}_{2}$ Swan system, Journal of Quantitative Spectroscopy and Radiative Transfer, 2013, vol. 124, p. 11

Brooke J. S. A., Ram R. S., Western C. M., Li G., Schwenke D. W., Bernath P. F., Einstein A Coefficients and Oscillator Strengths for the $\mathrm{A}^{2} \Pi-\mathrm{X}^{2} \Sigma^{+}$(Red) and $\mathrm{B}^{2} \Sigma^{+}-$ $\mathrm{X}^{2} \Sigma^{+}$(Violet) Systems and Rovibrational Transitions in the $\mathrm{X}^{2} \Sigma^{+}$State of CN, The Astrophysical Journal Supplement Series, 2014, vol. 210, p. 23

Carretta E., Bragaglia A., Gratton R., Lucatello S., Na-O anticorrelation and HB. VIII. Proton-capture elements and metallicities in 17 globular clusters from UVES spectra, A\&A, 2009a, vol. 505, p. 139

Carretta E., Bragaglia A., Gratton R. G., Lucatello S., VizieR Online Data Catalog: Abundances of red giants in 17 globular clusters (Carretta+, 2009), VizieR Online Data Catalog, 2009b, pp J/A+A/505/139

Carretta E., Bragaglia A., Gratton R. G., Recio-Blanco A., Lucatello S., D'Orazi V., Cassisi S., Properties of stellar generations in globular clusters and relations with global parameters, A\&A, 2010, vol. 516, p. A55 
Carretta E., Bragaglia A., Lucatello S., Gratton R. G., D’Orazi V., Sollima A., Aluminium abundances in five discrete stellar populations of the globular cluster NGC 2808, A\&A, 2018, vol. 615, p. A17

Chiappini C., The Formation and Evolution of the Milky Way, American Scientist, 2001, vol. 89 , p. 506

Coelho P., Percival S., Salaris M., Modelling chemical abundance anticorrelations on globular cluster spectra. In Astronomical Society of India Conference Series , vol. 6 of Astronomical Society of India Conference Series, 2012, p. 107

Coelho P., Percival S. M., Salaris M., Chemical Abundance Anticorrelations in Globular Cluster Stars: The Effect on Cluster Integrated Spectra, ApJ, 2011, vol. 734, p. 72

Coelho P. R. T., A new library of theoretical stellar spectra with scaled-solar and $\alpha$ enhanced mixtures, MNRAS, 2014, vol. 440, p. 1027

Coelho P. R. T., Bruzual G., Charlot S., To use or not to use synthetic stellar spectra in population synthesis models?, Monthly Notices of the Royal Astronomical Society, 2020, vol. 491 , p. 2025

Cruz M. A., Nucleosynthesis in extremely metal-poor and zero metallicity stars, LudwigMaximilians-Universitat Munchen, 2012, Tese de Doutorado

Czesla S., Schröter S., Schneider C. P., Huber K. F., Pfeifer F., Andreasen D. T., Zechmeister M., PyA: Python astronomy-related packages, Astrophysics Source Code Library, 2019, p. ascl:1906.010

de Souza Oliveira Filho K., de Fatima Oliveira Saraiva M., Astronomia e astrofisica. ${ }^{a}$ Ed. Universidade/UFRGS, 2004

Decressin T., Meynet G., Charbonnel C., Prantzos N., Ekström S., Fast rotating massive stars and the origin of the abundance patterns in galactic globular clusters, A\&A, 2007, vol. 464, p. 1029

Denissenkov P. A., Weiss A., Deep diffusive mixing in globular-cluster red giants., A\&A, 1996, vol. 308, p. 773 
Denissenkov P. A., Weiss A., A Contribution of ${ }^{26} \mathrm{Al}$ to the O-Al Anticorrelation in Globular Cluster Red Giants, The Astrophysical Journal, 2001, vol. 559, p. L115

D’Ercole A., D’Antona F., Ventura P., Vesperini E., McMillan S. L. W., Abundance patterns of multiple populations in globular clusters: a chemical evolution model based on yields from AGB ejecta, MNRAS, 2010, vol. 407, p. 854

D’Ercole A., Vesperini E., D'Antona F., McMillan S. L. W., Recchi S., Formation and dynamical evolution of multiple stellar generations in globular clusters, MNRAS, 2008, vol. 391, p. 825

Dias B., Barbuy B., Saviane I., Held E. V., Da Costa G. S., Ortolani S., Gullieuszik M., Vásquez S., FORS2/VLT survey of Milky Way globular clusters. II. Fe and Mg abundances of 51 Milky Way globular clusters on a homogeneous scale, A\&A, 2016, vol. 590, p. A9

Francis C., Anderson E., Two estimates of the distance to the Galactic Centre, MNRAS, 2014, vol. 441, p. 1105

Gonçalves G., Coelho P., Schiavon R., Usher C., How well can we determine ages and chemical abundances from spectral fitting of integrated light spectra?, arXiv e-prints, 2020, p. arXiv:2010.02940

Gräfener G., Koesterke L., Hamann W. R., Line-blanketed model atmospheres for WR stars, A\&A, 2002, vol. 387, p. 244

Gratton R., Bragaglia A., Carretta E., D’Orazi V., Lucatello S., Sollima A., What is a globular cluster? An observational perspective, A\&A Rev., 2019, vol. 27, p. 8

Gray R. O., SPECTRUM: A stellar spectral synthesis program, Astrophysics Source Code Library, 1999, p. ascl:9910.002

Grevesse N., Sauval A. J., Standard Solar Composition, Space Sci. Rev., 1998, vol. 85, p. 161

Gustafsson B., A Feautrier-Type Method for Model Atmospheres Including Convection, A\&A, 1971, vol. 10, p. 187 
Gustafsson B., Edvardsson B., Eriksson K., Jørgensen U. G., Nordlund Å., Plez B., A grid of MARCS model atmospheres for late-type stars. I. Methods and general properties, A\&A, 2008, vol. 486, p. 951

Hamann W. R., Gräfener G., A temperature correction method for expanding atmospheres, A\&A, 2003, vol. 410, p. 993

Hamann W. R., Schmutz W., Computed He II spectra for Wolf-Rayet stars - A grid of models, A\&A, 1987, vol. 174, p. 173

Harris W. E., A Catalog of Parameters for Globular Clusters in the Milky Way, AJ, 1996, vol. 112 , p. 1487

Hauschildt P. H., A fast operator perturbation method for the solution of the special relativistic equation of radiative transfer in spherical symmetry., J. Quant. Spec. Radiat. Transf., 1992, vol. 47, p. 433

Hauschildt P. H., Multi-level non-LTE radiative transfer in expanding shells., J. Quant. Spec. Radiat. Transf., 1993, vol. 50, p. 301

Hauschildt P. H., Baron E., Non-LTE treatment of Fe II in astrophysical plasmas., J. Quant. Spec. Radiat. Transf., 1995, vol. 54, p. 987

Hauschildt P. H., Baron E., Starrfield S., Allard F., The Effects of Fe II Non-LTE on Nova Atmospheres and Spectra, ApJ, 1996, vol. 462, p. 386

Hauschildt P. H., Lowenthal D. K., Baron E., Parallel Implementation of the PHOENIX Generalized Stellar Atmosphere Program. III. A Parallel Algorithm for Direct Opacity Sampling, The Astrophysical Journal Supplement Series, 2001, vol. 134, p. 323

Hauschildt P. H., Starrfield S., Shore S. N., Allard F., Baron E., The Physics of Early Nova Spectra, ApJ, 1995, vol. 447, p. 829

Herschel W., Astronomical Observations Relating to the Sidereal Part of the Heavens, and Its Connection with the Nebulous Part; Arranged for the Purpose of a Critical Examination, Philosophical Transactions of the Royal Society of London Series I, 1814, vol. 104 , p. 248 
Hinkle K., Wallace L., Valenti J., Harmer D., Visible and Near Infrared Atlas of the Arcturus Spectrum 3727-9300 A, 2000

Hubble Legacy Archive NGC 5927 Based on observations made with the NASA/ESA Hubble Space Telescope, and obtained from the Hubble Legacy Archive, which is a collaboration between the Space Telescope Science Institute (STScI/NASA), the Space Telescope European Coordinating Facility (ST-ECF/ESA) and the Canadian Astronomy Data Centre (CADC/NRC/CSA). https://upload.wikimedia.org/wikipedia/commons/ f/ff/NGC_5927_hst_11664_R815G555B390.png, acessado em 22-09-2020, 2016

Hubeny I., A computer program for calculating non-LTE model stellar atmospheres, Computer Physics Communications, 1988, vol. 52, p. 103

Hubeny I., Lanz T., Synspec: General Spectrum Synthesis Program, Astrophysics Source Code Library, 2011, p. ascl:1109.022

Jauregi U., Working notes on ATLAS 9 and SYNTHE, http://atmos.obspm.fr/images/ stories/download/working_notes.pdf, Acessado em: 18-05-2019., 2005

Jorgensen U. G., Larsson M., Iwamae A., Yu B., Line intensities for CH and their application to stellar atmospheres., A\&A, 1996, vol. 315, p. 204

Karakas A. I., Lattanzio J. C., The Dawes Review 2: Nucleosynthesis and Stellar Yields of Low- and Intermediate-Mass Single Stars, PASA, 2014, vol. 31, p. e030

Kraft R. P., Abundance Differences among Globular Cluster Giants: Primordial vs. Evolutionary Scenarios, PASP, 1994, vol. 106, p. 553

Kraft R. P., Sneden C., Smith G. H., Shetrone M. D., Langer G. E., Pilachowski C. A., Proton Capture Chains in Globular Cluster Stars.II.Oxygen, Sodium, Magnesium, and Aluminum Abundances in M13 Giants Brighter Than the Horizontal Branch, AJ, 1997, vol. 113, p. 279

Kurucz R., ATLAS9 Stellar Atmosphere Programs and $2 \mathrm{~km} / \mathrm{s}$ grid., ATLAS9 Stellar Atmosphere Programs and $2 \mathrm{~km} / \mathrm{s}$ grid. Kurucz CD-ROM No. 13. Cambridge, 1993, vol. 13 
Kurucz R. L., Atlas: a Computer Program for Calculating Model Stellar Atmospheres, SAO Special Report, 1970, vol. 309

Kurucz R. L., ATLAS12, SYNTHE, ATLAS9, WIDTH9, et cetera, Memorie della Societa Astronomica Italiana Supplementi, 2005a, vol. 8, p. 14

Kurucz R. L., New atlases for solar flux, irradiance, central intensity, and limb intensity, Memorie della Societa Astronomica Italiana Supplementi, 2005b, vol. 8, p. 189

Kurucz R. L., Including All the Lines: Data Releases for Spectra and Opacities through 2017. In Workshop on Astrophysical Opacities , vol. 515 of Astronomical Society of the Pacific Conference Series, 2018, p. 47

Kurucz R. L., Avrett E. H., Solar Spectrum Synthesis. I. A Sample Atlas from 224 to 300 nm, SAO Special Report, 1981, vol. 391

Kurucz R. L., Furenlid I., Sample Spectral Atlas for Sirius, SAO Special Report, 1979, vol. 387

Lasker B. M., Doggett J., McLean B., Sturch C., Djorgovski S., de Carvalho R. R., Reid I. N., The Palomar-ST ScI Digitized Sky Survey (POSS-II): Preliminary Data Availability, http://simbad.u-strasbg.fr/simbad/sim-basic?Ident=arcturus\&submit= SIMBAD+search. In Astronomical Data Analysis Software and Systems V, vol. 101 of Astronomical Society of the Pacific Conference Series http://alasky.u-strasbg.fr/ MocServer/query?id=CDS/P/DSS2/color\&get=record\&fmt=html, 1996, p. 88

Lee H., Worthey G., -Enhanced Integrated Lick/IDS Spectral Indices and Milky Way and M31 Globular Clusters and Early-Type Galaxies, The Astrophysical Journal Supplement Series, 2005, vol. 160, p. 176

Lee H.-c., Worthey G., $\alpha$-Enhanced Integrated Lick/IDS Spectral Indices and Milky Way and M31 Globular Clusters and Early-Type Galaxies, ApJS, 2005, vol. 160, p. 176

Lilly T., Alvensleben U. F.-V., Analysing globular cluster observations. Models and analysis tools for Lick/IDS indices, A\&A, 2006, vol. 457, p. 467

Martins L. P., Coelho P., Testing the accuracy of synthetic stellar libraries, MNRAS, 2007, vol. 381 , p. 1329 
Martins L. P., Lima-Dias C., Coelho P. R. T., Laganá T. F., Testing stellar population fitting ingredients with Globular Clusters I: Stellar libraries, MNRAS, 2019, vol. 484, p. 2388

Masseron T., Plez B., Van Eck S., Colin R., Daoutidis I., Godefroid M., Coheur P.-F., Bernath P., Jorissen A., Christlieb N., CH in stellar atmospheres: an extensive linelist, A\&A, 2014, vol. 571, p. A47

Meléndez J., The Sun. A typical star in the solar neighborhood?. In Setting the scene for Gaia and LAMOST, vol. 298 of IAU Symposium, 2014, p. 331

Meléndez J., Asplund M., Gustafsson B., Yong D., Ramírez I., Unprecedented accurate abundances: signatures of other Earths?. In Chemical Abundances in the Universe: Connecting First Stars to Planets , vol. 265 of IAU Symposium, 2010, p. 412

Mellinger A., A Color All-Sky Panorama Image of the Milky Way, PASP, 2009, vol. 121, p. 1180

Mészáros S., Allende Prieto C., Edvardsson B., Castelli F., García Pérez A. E., Gustafsson B., Majewski S. R., Plez B., Schiavon R., Shetrone M., de Vicente A., New ATLAS9 and MARCS Model Atmosphere Grids for the Apache Point Observatory Galactic Evolution Experiment (APOGEE), AJ, 2012, vol. 144, p. 120

Milone A. P., Marino A. F., Renzini A., D'Antona F., Anderson J., Barbuy B., Bedin L. R., Bellini A., Brown T. M., Cassisi S., Cordoni G., Lagioia E. P., Nardiello D., Ortolani S., Piotto G., Sarajedini A., Tailo M., et al. The Hubble Space Telescope UV legacy survey of galactic globular clusters - XVI. The helium abundance of multiple populations, MNRAS, 2018, vol. 481, p. 5098

Milone A. P., Piotto G., Renzini A., Marino A. F., Bedin L. R., Vesperini E., D’Antona F., Nardiello D., Anderson J., King I. R., Yong D., Bellini A., Aparicio A., Barbuy B., Brown T. M., Cassisi S., Ortolani S., Salaris M., Sarajedini A., van der Marel R. P., The Hubble Space Telescope UV Legacy Survey of Galactic globular clusters - IX. The Atlas of multiple stellar populations, Monthly Notices of the Royal Astronomical Society, 2016, vol. 464, p. 3636 
Mura-Guzmán A., Villanova S., Muñoz C., Tang B., Chemical study of the metal-rich globular cluster NGC 5927, MNRAS, 2018, vol. 474, p. 4541

NASA SDO/AIA 304, https://sdo.gsfc.nasa.gov/assets/img/browse/2010/08/19/ 20100819_003221_4096_0304.jpg, 2010

NASA/ESA/STScI/AURA/ESA-HST and the Hubble Heritage (STScI/AURA)ESA/Hubble Collaboration Acknowledgment: J. Mack (STScI) and G. Piotto (University of Padova, Italy), Globular cluster 47 Tucanae, https: //www.spacetelescope.org/images/heic1510a/, acessado em 22-09-2020, 2015

NASA/STScI Globular Cluster Messier 79 (M79, NGC 1904), NAS5-03127, https:// hubblesite.org/image/4092/gallery, acessado em 22-09-2020, 2017

Nordlander T., Bessell M. S., Da Costa G. S., Mackey A. D., Asplund M., Casey A. R., Chiti A., Ezzeddine R., Frebel A., Lind K., Marino A. F., Murphy S. J., Norris J. E., Schmidt B. P., Yong D., The lowest detected stellar Fe abundance: the halo star SMSS J160540.18-144323.1, MNRAS, 2019, vol. 488, p. L109

Peebles P. J. E., Discovery of the hot Big Bang: What happened in 1948, European Physical Journal H, 2014, vol. 39, p. 205

Percival S. M., Salaris M., Cassisi S., Pietrinferni A., A Large Stellar Evolution Database for Population Synthesis Studies. IV. Integrated Properties and Spectra, ApJ, 2009, vol. 690 , p. 427

Pietrinferni A., Cassisi S., Salaris M., Castelli F., A Large Stellar Evolution Database for Population Synthesis Studies. II. Stellar Models and Isochrones for an $\alpha$-enhanced Metal Distribution, ApJ, 2006, vol. 642, p. 797

Piotto G., Bedin L. R., Anderson J., King I. R., Cassisi S., Milone A. P., Villanova S., Pietrinferni A., Renzini A., A Triple Main Sequence in the Globular Cluster NGC 2808, ApJ, 2007, vol. 661, p. L53

Piotto G., King I. R., Djorgovski S. G., Sosin C., Zoccali M., Saviane I., De Angeli F., Riello M., Recio-Blanco A., Rich R. M., Meylan G., Renzini A., HST color-magnitude 
diagrams of 74 galactic globular clusters in the HST F439W and F555W bands, A\&A, 2002, vol. 391, p. 945

Piotto G., Milone A. P., Bedin L. R., Anderson J., King I. R., Marino A. F., Nardiello D., Aparicio A., Barbuy B., Bellini A., Brown T. M., Cassisi S., Cool A. M., Cunial A., Dalessandro E., D’Antona F., Ferraro F. R., Hidalgo S., Lanzoni B., et al., The Hubble Space Telescope UV Legacy Survey of Galactic Globular Clusters. I. Overview of the Project and Detection of Multiple Stellar Populations, AJ, 2015, vol. 149, p. 91

Pols O., Close Binary Progenitors of Type Ib/Ic and IIb/II-L Supernovae. In The Third Pacific Rim Conference on Recent Development on Binary Star Research, vol. 130 of Astronomical Society of the Pacific Conference Series, 1997, p. 153

Proctor R. N., Forbes D. A., Beasley M. A., A robust method for the analysis of integrated spectra from globular clusters using Lick indices, Monthly Notices of the Royal Astronomical Society, 2004, vol. 355, p. 1327

Queiroz A. B. A., Anders F., Chiappini C., Khalatyan A., Santiago B. X., Steinmetz M., Valentini M., Miglio A., Bossini D., Barbuy B., Minchev I., Minniti D., García Hernández D. A., Schultheis M., Beaton R. L., Beers T. C., et al., From the bulge to the outer disc: StarHorse stellar parameters, distances, and extinctions for stars in APOGEE DR16 and other spectroscopic surveys, A\&A, 2020, vol. 638, p. A76

Ram R., Engleman R., Bernath P., Fourier Transform Emission Spectroscopy of theA2-X2 Transition of SiH and SiD, Journal of Molecular Spectroscopy, 1998, vol. 190, p. 341

Ramírez I., Prieto C. A., Fundamental Parameters and Chemical Composition of Arcturus, ApJ, 2011, vol. 743, p. 135

Renzini A., Finding forming globular clusters at high redshifts, MNRAS, 2017, vol. 469, p. L63

Rodríguez-Merino L. H., Mayya Y. D., Coelho P. R. T., Bruzual G., Charlot S., Carrasco E., de Paz A. G., High-resolution Spectral Line Indices Useful for the Analysis of Stellar Populations, The Astrophysical Journal, 2020, vol. 889, p. L31 
Salaris M., Cassisi S., Weiss A., Red Giant Branch Stars: The Theoretical Framework, PASP, 2002, vol. 114, p. 375

Sandage A., Main-sequence photometry, color-magnitude diagrams, and ages for the globular clusters M3, M13, M15 and M92., ApJ, 1970, vol. 162, p. 841

Sander A., Shenar T., Hainich R., Gímenez-García A., Todt H., Hamann W. R., On the consistent treatment of the quasi-hydrostatic layers in hot star atmospheres, A\&A, 2015, vol. 577 , p. A13

Sbordone L., Kurucz's codes under GNU-Linux, Memorie della Societa Astronomica Italiana Supplementi, 2005, vol. 8, p. 61

Sbordone L., Bonifacio P., Atlas CookBook, http://atmos.obspm.fr/index.php/ documentation/7, Acessado em: 18-05-2019., 2005

Sbordone L., Bonifacio P., Castelli F., Kurucz R. L., ATLAS and SYNTHE under Linux, Memorie della Societa Astronomica Italiana Supplementi, 2004, vol. 5, p. 93

Schiavon R. P., Rose J. A., Courteau S., MacArthur L. A., A Library of Integrated Spectra of Galactic Globular Clusters, ApJS, 2005, vol. 160, p. 163

Schwarzschild M., On the Helium Content of the Sun., ApJ, 1946, vol. 104, p. 203

Spite M., Étude de l'étoile $\chi$ Draconis, Annales d'Astrophysique, 1967, vol. 30, p. 211

Tagirov R. V., Shapiro A. I., Schmutz W., NESSY: NLTE spectral synthesis code for solar and stellar atmospheres, A\&A, 2017, vol. 603, p. A27

Thomas D., Johansson J., Maraston C., Chemical abundance ratios of galactic globular clusters from modelling integrated light spectroscopy, MNRAS, 2011, vol. 412, p. 2199

Thomas D., Maraston C., Bender R., Stellar population models of Lick indices with variable element abundance ratios, Monthly Notices of the Royal Astronomical Society, 2003, vol. 339 , p. 897

Thomas D., Maraston C., Johansson J., Flux-calibrated stellar population models of Lick absorption-line indices with variable element abundance ratios, MNRAS, 2011, vol. 412, p. 2183 
Tsantaki M., Andreasen D. T., Teixeira G. D. C., Sousa S. G., Santos N. C., Delgado-Mena E., Bruzual G., Atmospheric stellar parameters for large surveys using FASMA, a new spectral synthesis package, MNRAS, 2018, vol. 473, p. 5066

Usher C., Pastorello N., Bellstedt S., Alabi A., Cerulo P., Chevalier L., Fraser-McKelvie A., Penny S., Foster C., McDermid R. M., Schiavon R. P., Villaume A., The WAGGS project - I. The WiFeS Atlas of Galactic Globular cluster Spectra (DR1: https://docs. datacentral.org.au/waggs/), MNRAS, 2017, vol. 468, p. 3828

Vazdekis A., Arimoto N., A Robust Age Indicator for Old Stellar Populations, The Astrophysical Journal, 1999, vol. 525, p. 144

Vazdekis A., Sánchez-Blázquez P., Falcón-Barroso J., Cenarro A. J., Beasley M. A., Cardiel N., Gorgas J., Peletier R. F., Evolutionary stellar population synthesis with MILES - I. The base models and a new line index system, MNRAS, 2010, vol. 404, p. 1639

Ventura P., Dell'Agli F., Lugaro M., Romano D., Tailo M., Yagüe A., Gas and dust from metal-rich AGB stars, A\&A, 2020, vol. 641, p. A103

Virtanen P., Gommers R., Oliphant T. E., Haberland M., Reddy T., Cournapeau D., Burovski E., Peterson P., Weckesser W., et al. SciPy 1.0: fundamental algorithms for scientific computing in Python, Nature Methods, 2020, vol. 17, p. 261-272

Walcher C. J., Coelho P., Gallazzi A., Charlot S., Differential stellar population models: how to reliably measure $[\mathrm{Fe} / \mathrm{H}]$ and $[\alpha / \mathrm{Fe}]$ in galaxies, MNRAS, 2009, vol. 398, p. L44

Wallace L., Hinkle K. H., Livingston W. C., Davis S. P., An Optical and Near-infrared (2958-9250 A) Solar Flux Atlas, ApJS, 2011, vol. 195, p. 6

Wheeler J. C., Sneden C., Truran James W. J., Abundance ratios as a function of metallicity., ARA\&A, 1989, vol. 27, p. 279

Worthey G., Faber S. M., Gonzalez J. J., Burstein D., Old Stellar Populations. V. Absorption Feature Indices for the Complete Lick/IDS Sample of Stars, ApJS, 1994, vol. 94, p. 687

Worthey G., Ottaviani D. L., $\mathrm{H} \gamma$ and $\mathrm{H} \delta$ Absorption Features in Stars and Stellar Populations, ApJS, 1997, vol. 111, p. 377 
Yousefi M., Bernath P. F., Hodges J., Masseron T., A new line list for the A2+-X2 electronic transition of $\mathrm{OH}$, Journal of Quantitative Spectroscopy and Radiative Transfer, 2018, vol. 217, p. 416 
Apêndice 

Apêndice A

\section{Abundâncias estelares}

\section{A.1 Sol}

\section{A.1.1 Grevesse e Sauval (1998)}

ABUNDANCE SCALE $\quad 1.00000$ ABUNDANCE CHANGE 10.9204020 .07834

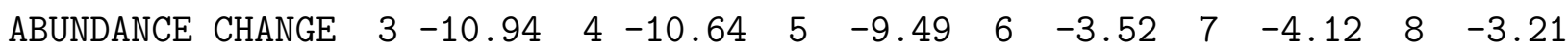

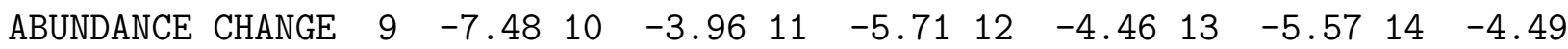

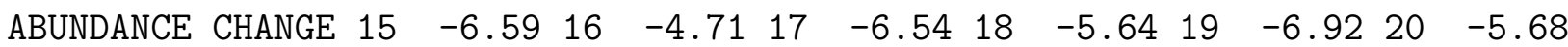

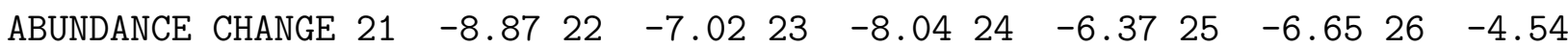

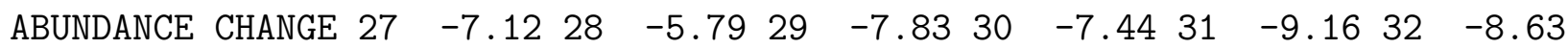

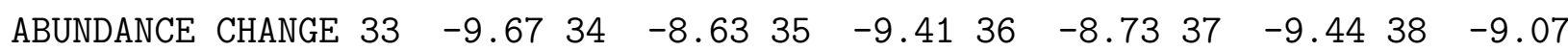

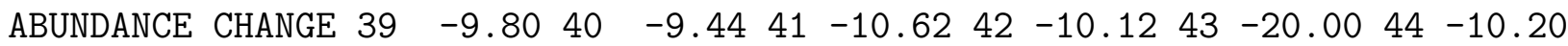

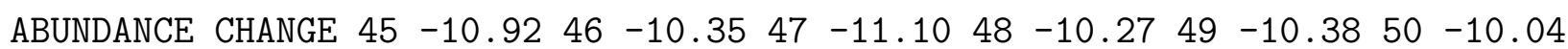

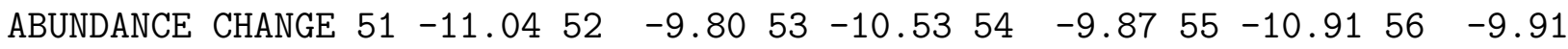

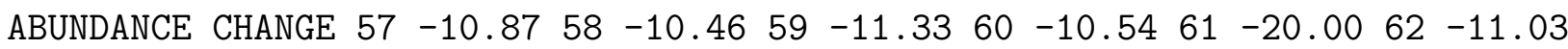

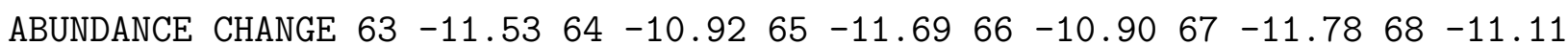

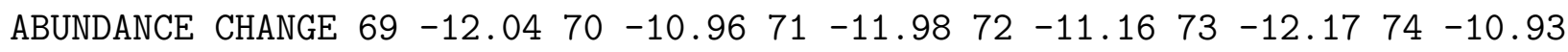

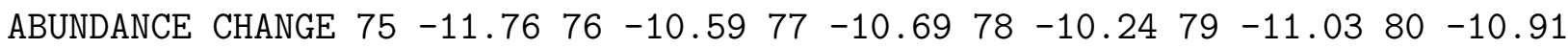

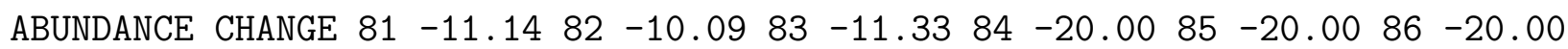

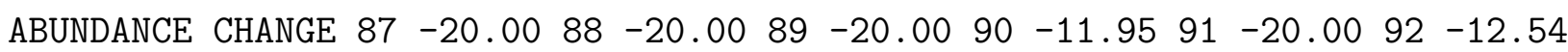

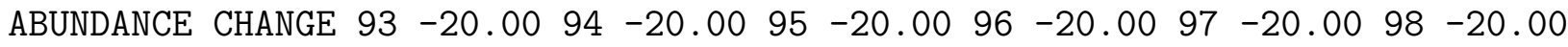
ABUNDANCE CHANGE $99-20.00$ 


\section{A.1.2 Asplund et al. 2005)}

ABUNDANCE SCALE $\quad 1.00000$ ABUNDANCE CHANGE $1 \quad 0.9208020 .07837$

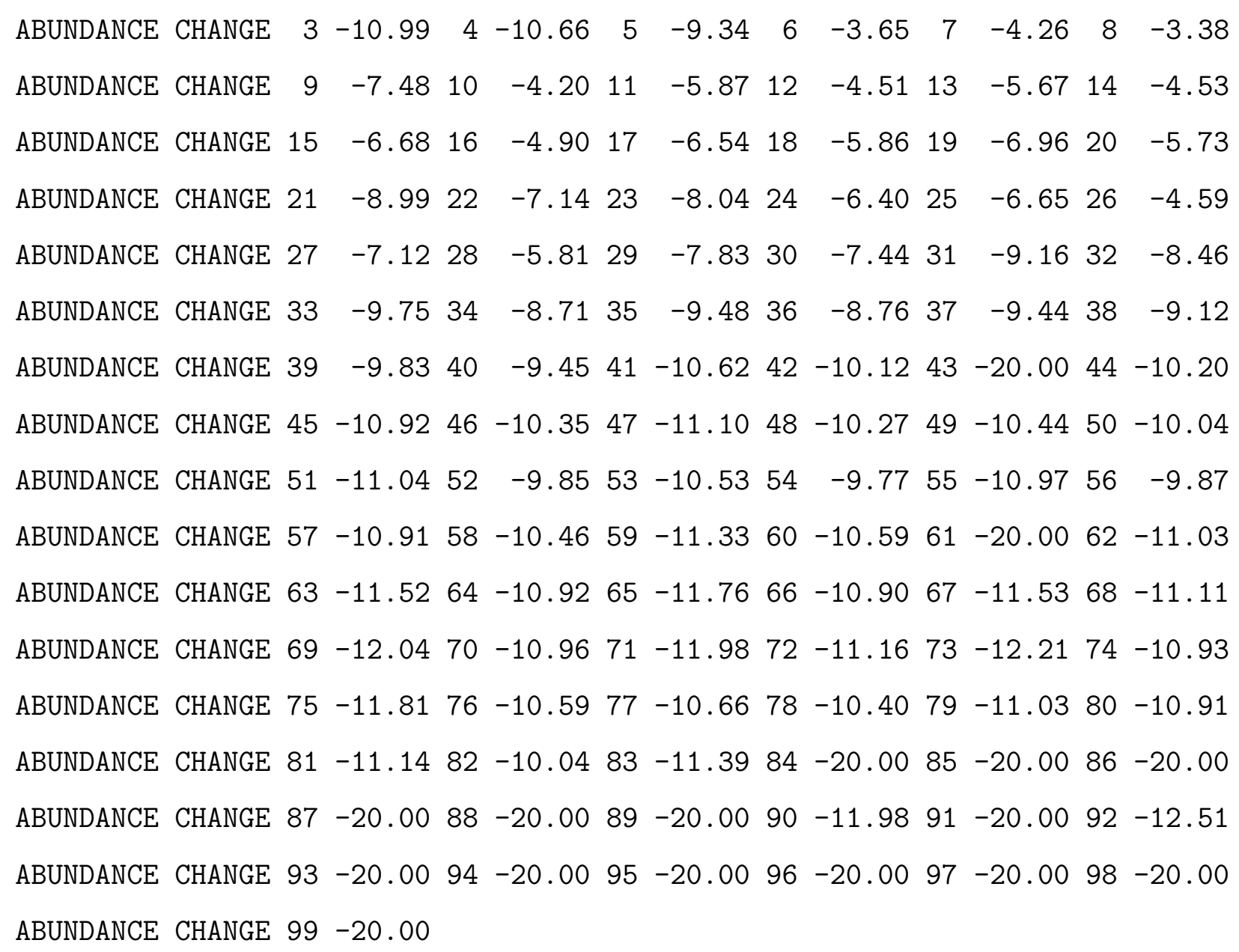




\section{A.1.3 Asplund et al. 2009)}

ABUNDANCE SCALE $\quad 1.00000$ ABUNDANCE CHANGE 10.9206820 .07836

$\begin{array}{lllllllllllll}\text { ABUNDANCE CHANGE } & 3 & -10.99 & 4 & -10.66 & 5 & -9.34 & 6 & -3.61 & 7 & -4.21 & 8 & -3.35 \\ \text { ABUNDANCE CHANGE } & 9 & -7.48 & 10 & -4.11 & 11 & -5.80 & 12 & -4.44 & 13 & -5.59 & 14 & -4.53 \\ \text { ABUNDANCE CHANGE } & 15 & -6.63 & 16 & -4.92 & 17 & -6.54 & 18 & -5.64 & 19 & -7.01 & 20 & -5.70 \\ \text { ABUNDANCE CHANGE } & 21 & -8.89 & 22 & -7.09 & 23 & -8.11 & 24 & -6.40 & 25 & -6.61 & 26 & -4.54 \\ \text { ABUNDANCE CHANGE } & 27 & -7.05 & 28 & -5.82 & 29 & -7.85 & 30 & -7.48 & 31 & -9.00 & 32 & -8.39 \\ \text { ABUNDANCE CHANGE } & 33 & -9.74 & 34 & -8.70 & 35 & -9.50 & 36 & -8.79 & 37 & -9.52 & 38 & -9.17 \\ \text { ABUNDANCE CHANGE } & 39 & -9.83 & 40 & -9.46 & 41 & -10.58 & 42 & -10.16 & 43 & -20.00 & 44 & -10.29 \\ \text { ABUNDANCE CHANGE } & 45 & -11.13 & 46 & -10.47 & 47 & -11.10 & 48 & -10.33 & 49 & -11.24 & 50 & -10.00 \\ \text { ABUNDANCE CHANGE } & 51 & -11.03 & 52 & -9.86 & 53 & -10.49 & 54 & -9.80 & 55 & -10.96 & 56 & -9.86 \\ \text { ABUNDANCE CHANGE } & 57 & -10.94 & 58 & -10.46 & 59 & -11.32 & 60 & -10.62 & 61 & -20.00 & 62 & -11.08 \\ \text { ABUNDANCE CHANGE } & 63 & -11.52 & 64 & -10.97 & 65 & -11.74 & 66 & -10.94 & 67 & -11.56 & 68 & -11.12 \\ \text { ABUNDANCE CHANGE } & 69 & -11.94 & 70 & -11.20 & 71 & -11.94 & 72 & -11.19 & 73 & -12.16 & 74 & -11.19 \\ \text { ABUNDANCE CHANGE } & 75 & -11.78 & 76 & -10.64 & 77 & -10.66 & 78 & -10.42 & 79 & -11.12 & 80 & -10.87 \\ \text { ABUNDANCE CHANGE } & 81 & -11.14 & 82 & -10.29 & 83 & -11.39 & 84 & -20.00 & 85 & -20.00 & 86 & -20.00 \\ \text { ABUNDANCE CHANGE } & 87 & -20.00 & 88 & -20.00 & 89 & -20.00 & 90 & -12.02 & 91 & -20.00 & 92 & -12.58 \\ \text { ABUNDANCE CHANGE } & 93 & -20.00 & 94 & -20.00 & 95 & -20.00 & 96 & -20.00 & 97 & -20.00 & 98 & -20.00 \\ \text { ABUNDANCE CHANGE } & 99 & -20.00 & & & & & & & & & & \end{array}$




\section{A.2 Arcturus}

\section{A.2.1 Ramírez e Prieto (2011)}

ABUNDANCE SCALE $\quad 0.30200$ ABUNDANCE CHANGE 10.9110020 .08900

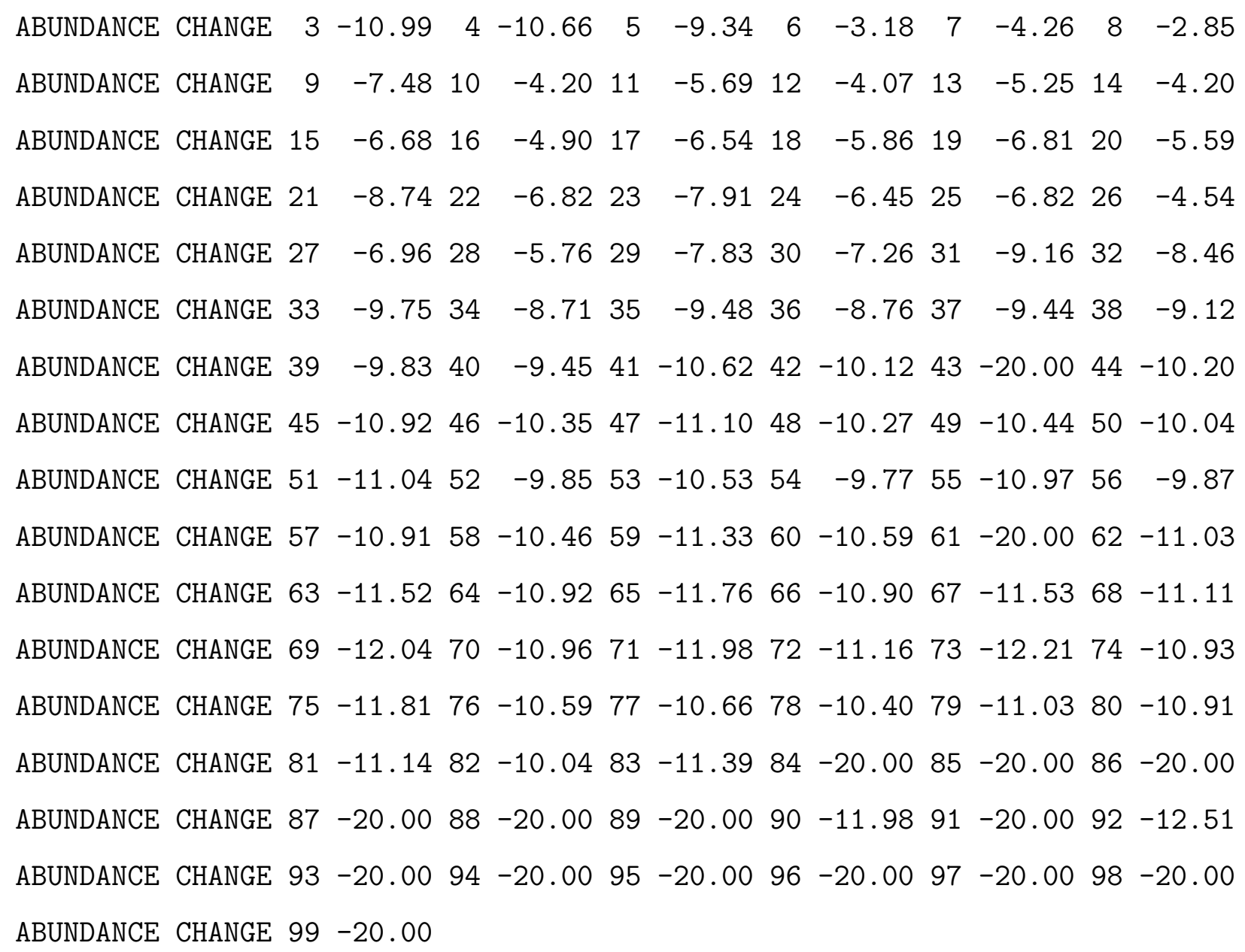




\section{Apêndice B}

\section{Cobertura de parâmetros da grade}

\section{B.1 NGC 1904}

Tabela B.1 - Cobertura de parâmetros e quantidade de estrelas (\#) de cada par $\left\{T_{\text {eff }}, \log g\right\}$ para NGC 1904, com total de 76 pares e 10929 estrelas.

\begin{tabular}{|c|c|c|c|c|c|c|c|c|c|c|c|}
\hline$T_{\text {eff }}(\mathbf{K})$ & $\log g$ & $\#$ & $T_{\text {eff }}(\mathbf{K})$ & $\log g$ & $\#$ & $T_{\text {eff }}(\mathbf{K})$ & $\log g$ & $\#$ & $T_{\text {eff }}(\mathbf{K})$ & $\log g$ & $\#$ \\
\hline 3800,0 & 1,0 & 1 & 4500,0 & 1,5 & 11 & 4500,0 & 2,0 & 7 & 4500,0 & 4,5 & 10 \\
\hline 4750,0 & 1,5 & 4 & 4750,0 & 2,0 & 30 & 4750,0 & 2,5 & 35 & 4750,0 & 4,5 & 96 \\
\hline 5000,0 & 1,5 & 1 & 5000,0 & 2,0 & 7 & 5000,0 & 2,5 & 54 & 5000,0 & 3,0 & 107 \\
\hline 5000,0 & 3,5 & 29 & 5000,0 & 4,5 & 416 & 5250,0 & 2,0 & 3 & 5250,0 & 2,5 & 5 \\
\hline 5250,0 & 3,0 & 52 & 5250,0 & 3,5 & 349 & 5250,0 & 4,0 & 112 & 5250,0 & 4,5 & 842 \\
\hline 5250,0 & 5,0 & 3 & 5500,0 & 2,5 & 1 & 5500,0 & 3,5 & 28 & 5500,0 & 4,0 & 371 \\
\hline 5500,0 & 4,5 & 1294 & 5500,0 & 5,0 & 12 & 5750,0 & 2,5 & 3 & 5750,0 & 4,0 & 572 \\
\hline 5750,0 & 4,5 & 1724 & 5750,0 & 5,0 & 55 & 6000,0 & 2,5 & 1 & 6000,0 & 4,0 & 548 \\
\hline 6000,0 & 4,5 & 1870 & 6000,0 & 5,0 & 78 & 6250,0 & 4,0 & 117 & 6250,0 & 4,5 & 1339 \\
\hline 6250,0 & 5,0 & 92 & 6500,0 & 4,5 & 382 & 6500,0 & 5,0 & 104 & 6750,0 & 3,0 & 1 \\
\hline 6750,0 & 4,5 & 61 & 7000,0 & 4,5 & 6 & 7250,0 & 3,0 & 2 & 7500,0 & 3,0 & 3 \\
\hline 7750,0 & 3,0 & 5 & 7750,0 & 3,5 & 1 & 8000,0 & 3,0 & 1 & 8000,0 & 3,5 & 7 \\
\hline 8250,0 & 3,5 & 11 & 8500,0 & 3,5 & 5 & 8750,0 & 3,5 & 9 & 9000,0 & 3,5 & 2 \\
\hline 9250,0 & 3,5 & 2 & 9250,0 & 4,0 & 1 & 9500,0 & 3,5 & 6 & 9500,0 & 4,0 & 1 \\
\hline 9750,0 & 3,5 & 2 & 9750,0 & 4,0 & 2 & 10000,0 & 4,0 & 2 & 10250,0 & 3,5 & 2 \\
\hline 10250,0 & 4,0 & 2 & 10500,0 & 4,0 & 1 & 10750,0 & 4,0 & 3 & 11000,0 & 4,0 & 2 \\
\hline 11250,0 & 4,0 & 2 & 11750,0 & 3,5 & 1 & 11750,0 & 4,0 & 2 & 12000,0 & 4,0 & 3 \\
\hline 12250,0 & 4,0 & 5 & 12500,0 & 4,0 & 2 & 12750,0 & 4,0 & 2 & 12750,0 & 4,5 & 1 \\
\hline 13000,0 & 4,0 & 1 & 13000,0 & 4,5 & 1 & 14000,0 & 4,0 & 2 & 14000,0 & 4,5 & 2 \\
\hline
\end{tabular}




\section{B.2 NGC 0104}

Tabela B.2 - Cobertura de parâmetros e quantidade de estrelas (\#) de cada par $\left\{T_{\text {eff }}, \log g\right\}$ para NGC 0104, com total de 52 pares e 24594 estrelas.

\begin{tabular}{|c|c|c||c|c|c||c|c|c|}
\hline$T_{\text {eff }}(\mathbf{K})$ & $\log g$ & $\#$ & $T_{\text {eff }}(\mathbf{K})$ & $\log g$ & $\#$ & $\left.T_{\text {eff }} \mathbf{K}\right)$ & $\log g$ & $\#$ \\
\hline 3800,0 & 1,0 & 7 & 4000,0 & 1,0 & 1 & 4000,0 & 1,5 & 8 \\
\hline 4250,0 & 1,5 & 8 & 4250,0 & 4,5 & 8 & 4500,0 & 1,5 & 7 \\
\hline 4500,0 & 2,0 & 73 & 4500,0 & 2,5 & 90 & 4500,0 & 4,5 & 229 \\
\hline 4750,0 & 2,0 & 42 & 4750,0 & 2,5 & 223 & 4750,0 & 3,0 & 276 \\
\hline 4750,0 & 3,5 & 35 & 4750,0 & 4,0 & 171 & 4750,0 & 4,5 & 1076 \\
\hline 5000,0 & 2,0 & 23 & 5000,0 & 2,5 & 280 & 5000,0 & 3,0 & 113 \\
\hline 5000,0 & 3,5 & 889 & 5000,0 & 4,0 & 637 & 5000,0 & 4,5 & 2134 \\
\hline 5000,0 & 5,0 & 1 & 5250,0 & 2,5 & 48 & 5250,0 & 3,5 & 50 \\
\hline 5250,0 & 4,0 & 947 & 5250,0 & 4,5 & 3068 & 5250,0 & 5,0 & 8 \\
\hline 5500,0 & 2,5 & 2 & 5500,0 & 3,0 & 1 & 5500,0 & 4,0 & 1871 \\
\hline 5500,0 & 4,5 & 3530 & 5500,0 & 5,0 & 34 & 5750,0 & 4,0 & 2352 \\
\hline 5750,0 & 4,5 & 3608 & 5750,0 & 5,0 & 20 & 6000,0 & 4,0 & 363 \\
\hline 6000,0 & 4,5 & 1967 & 6000,0 & 5,0 & 20 & 6250,0 & 4,0 & 23 \\
\hline 6250,0 & 4,5 & 264 & 6250,0 & 5,0 & 17 & 6500,0 & 4,0 & 7 \\
\hline 6500,0 & 4,5 & 37 & 6750,0 & 4,0 & 7 & 6750,0 & 4,5 & 2 \\
\hline 7000,0 & 4,0 & 7 & 7000,0 & 4,5 & 2 & 7250,0 & 3,5 & 2 \\
\hline 7250,0 & 4,0 & 2 & 7500,0 & 4,0 & 2 & 7750,0 & 4,0 & 1 \\
\hline 8000,0 & 4,5 & 1 & & & & & & \\
\hline
\end{tabular}




\section{B.3 NGC 5927}

Tabela B.3 - Cobertura de parâmetros e quantidade de estrelas (\#) de cada par $\left\{T_{\text {eff }}, \log g\right\}$ para NGC 5927, com total de 59 pares e 13594 estrelas.

\begin{tabular}{|c|c|c||c|c|c||c|c|c|c||c|c|c|}
\hline$T_{\text {eff }}(\mathbf{K})$ & $\log g$ & $\#$ & $T_{\text {eff }}(\mathbf{K})$ & $\log g$ & $\#$ & $T_{\text {eff }}(\mathbf{K})$ & $\log g$ & $\#$ & $T_{\text {eff }}(\mathbf{K})$ & $\log g$ & $\#$ \\
\hline 3800,0 & 1,5 & 1 & 4000,0 & 1,0 & 1 & 4000,0 & 1,5 & 13 & 4250,0 & 1,5 & 4 \\
\hline 4250,0 & 2,0 & 1 & 4250,0 & 4,5 & 25 & 4500,0 & 2,0 & 20 & 4500,0 & 2,5 & 226 \\
\hline 4500,0 & 3,0 & 59 & 4500,0 & 3,5 & 9 & 4500,0 & 4,5 & 250 & 4750,0 & 2,0 & 11 \\
\hline 4750,0 & 2,5 & 109 & 4750,0 & 3,0 & 129 & 4750,0 & 3,5 & 452 & 4750,0 & 4,0 & 853 \\
\hline 4750,0 & 4,5 & 711 & 5000,0 & 2,5 & 24 & 5000,0 & 3,0 & 8 & 5000,0 & 3,5 & 107 \\
\hline 5000,0 & 4,0 & 1167 & 5000,0 & 4,5 & 1182 & 5000,0 & 5,0 & 1 & 5250,0 & 3,0 & 6 \\
\hline 5250,0 & 3,5 & 27 & 5250,0 & 4,0 & 1712 & 5250,0 & 4,5 & 1427 & 5250,0 & 5,0 & 19 \\
\hline 5500,0 & 2,5 & 2 & 5500,0 & 3,0 & 6 & 5500,0 & 3,5 & 15 & 5500,0 & 4,0 & 1350 \\
\hline 5500,0 & 4,5 & 1498 & 5500,0 & 5,0 & 75 & 5750,0 & 3,0 & 8 & 5750,0 & 3,5 & 7 \\
\hline 5750,0 & 4,0 & 287 & 5750,0 & 4,5 & 941 & 5750,0 & 5,0 & 107 & 6000,0 & 2,5 & 1 \\
\hline 6000,0 & 3,0 & 1 & 6000,0 & 3,5 & 4 & 6000,0 & 4,0 & 25 & 6000,0 & 4,5 & 348 \\
\hline 6000,0 & 5,0 & 223 & 6250,0 & 2,5 & 1 & 6250,0 & 3,0 & 1 & 6250,0 & 3,5 & 1 \\
\hline 6250,0 & 4,0 & 10 & 6250,0 & 4,5 & 91 & 6500,0 & 2,5 & 1 & 6500,0 & 3,0 & 1 \\
\hline 6500,0 & 3,5 & 1 & 6500,0 & 4,0 & 5 & 6500,0 & 4,5 & 17 & 6750,0 & 4,0 & 4 \\
\hline 6750,0 & 4,5 & 7 & 7000,0 & 4,5 & 1 & 8000,0 & 4,0 & 1 & & & \\
\hline
\end{tabular}


Apêndice C

\section{Comparação com as obervações}

\section{C.1 ADEV em relação a WAGGS}

Tabela C.1 - Comparação entre os ADEVs calculados com os espectros convoluídos em relação ao espectro observado por WAGGS, nas regiões do Índice de Lick e intervalo lambda com início correspondente ao estudo da nova lista atômica e com fim ao espectro observado por WAGGS (Usher et al. 2017).

\begin{tabular}{|c|c|c|c|c|}
\hline NGC & ID & Região $(\AA)$ & $\mathrm{Ger}_{1}$ & $\mathrm{Ger}_{2}$ \\
\hline 1904 & Completo & 3726,702 a 9049.554 & 0.02924 & 0.03053 \\
\hline 1904 & $\mathrm{CN}_{1,2}$ & 4080,125 a 4284,125 & 0.02825 & 0.02416 \\
\hline 0104 & $\mathrm{Ca} 4227$ & 4211,000 a 4251,000 & 0.02271 & 0.01857 \\
\hline 0104 & G4300 & 4266,375 a & 0.04135 & 0.02490 \\
\hline 0104 & $\mathrm{Na} D$ & 5860,625 a 5948,125 & 0.01741 & 0.01623 \\
\hline 0104 & Com & 3726,702 & 0.06094 & 0.06389 \\
\hline 0104 & $\mathrm{CN}_{1,2}$ & 4080,125 & 0.05726 & 0.04012 \\
\hline 0104 & $\mathrm{Ca} 4227$ & $4211,000 \mathrm{a}$ & 0.04956 & 0.03039 \\
\hline 0104 & G4300 & 4266,375 a & 0.05392 & 0.03462 \\
\hline 0104 & $\mathrm{Na} D$ & 5860,625 a 5948,125 & 0.02909 & 0.02245 \\
\hline 5927 & Completo & 3726,702 a 9049.554 & 0.68319 & 0.68716 \\
\hline 5927 & $\mathrm{CN}_{1,2}$ & 4080,125 a 4284,125 & 0.09454 & 0.07693 \\
\hline 5927 & $\mathrm{Ca} 4227$ & 4211,000 a 4251,000 & 0.06723 & 0.04773 \\
\hline 5927 & G4300 & 4266,375 с & 0.05282 & 0.04270 \\
\hline 5927 & $\mathrm{Na} D$ & 5860,625 a 5948,125 & 0.11707 & 0.09101 \\
\hline
\end{tabular}


